DESY 99-020

NTZ 16/99

March 1999

\title{
The Virtual Compton Amplitude in the Generalized Bjorken Region: Twist-2 Contributions
}

\author{
Johannes Blümlein ${ }^{a}$, Bodo Geyer $^{b}$, and Dieter Robaschik ${ }^{a, c}$ \\ ${ }^{a}$ DESY - Zeuthen, Platanenallee 6, D-15735 Zeuthen, Germany \\ ${ }^{b}$ Naturwissenschaftlich-Theoretisches Zentrum, Universität Leipzig, \\ Augustusplatz 10, D-04109 Leipzig, Germany \\ ${ }^{c}$ Institut für Theoretische Physik, Karl-Franzens-Universität Graz, \\ Univeristätsplatz 5, A-8010 Graz, Austria \\ October 2, 2018
}

\begin{abstract}
A systematic derivation is presented of the twist-2 anomalous dimensions of the general quark and gluon light-ray operators in the generalized Bjorken region in leading order both for unpolarized and polarized scattering. Various representations of the anomalous dimensions are derived in the non-local and local light cone expansion and their properties are discussed in detail. Evolution equations for these operators are derived using different representations. General two- and single-variable evolution equations are presented for the expectation values of these operators for non-forward scattering. The Compton amplitude is calculated in terms of these distribution amplitudes. In the limit of forward scattering a new derivation of the integral relations between the twist -2 contributions to the structure functions is given. Special limiting cases which are derived from the general relations are discussed, as the forward case, near-forward scattering, and vacuum-meson transition. Solutions of the two-variable evolution equations for non-forward scattering are presented.
\end{abstract}




\section{Introduction}

The Compton amplitude for the scattering of a virtual photon off a hadron

$$
\gamma^{*}+p_{1} \rightarrow \gamma^{*}+p_{2}
$$

provides one of the basic tools to understand the short-distance behavior of the nucleon and to test Quantum Chromodynamics (QCD) at large space-like virtualities. The Compton amplitude for the general case of non-forward scattering is given by

$$
T_{\mu \nu}\left(p_{+}, p_{-}, q\right)=i \int d^{4} x e^{i q x}\left\langle p_{2}, S_{2}\left|T\left(J_{\mu}(x / 2) J_{\nu}(-x / 2)\right)\right| p_{1}, S_{1}\right\rangle .
$$

Here,

$$
\begin{aligned}
p_{+}=p_{2}+p_{1}, & p_{-}=p_{2}-p_{1}=q_{1}-q_{2}, \\
q=\frac{1}{2}\left(q_{1}+q_{2}\right), & p_{1}+q_{1}=p_{2}+q_{2},
\end{aligned}
$$

where $q_{1}\left(q_{2}\right)$ and $p_{1}\left(p_{2}\right)$ denote the four-momenta of the incoming (outgoing) photon and hadron, respectively, and $S_{1}, S_{2}$ are the spins of the initial- and final-state hadron. The generalized Bjorken region is defined by the conditions

$$
\nu=q p_{+} \longrightarrow \infty, \quad Q^{2}=-q^{2} \longrightarrow \infty
$$

keeping the variables

$$
\xi=\frac{Q^{2}}{q p_{+}}, \quad \eta=\frac{q p_{-}}{q p_{+}}=\frac{q_{1}^{2}-q_{2}^{2}}{2 \nu}
$$

fixed. Relations between these kinematic variables are given in Appendix A For $q_{2}^{2}=0$ one obtains $\eta=-\xi$. In distinction to deep inelastic forward scattering, the scaling variable $\xi$ is not restricted to the interval $0 \leq \xi \leq 1$. For forward scattering $q=q_{1}=q_{2}, p_{+}=2 p, \eta=0$, $\xi \rightarrow x_{\mathrm{Bj}}=Q^{2} /(2 p q)$, holds. In principle the results presented below can be analytically continued to time-like processes with two space-like initial state photons as a necessary condition.

The (renormalized) time-ordered product in Eq. (1.2) can be represented in terms of the operator product expansion. Unlike the local operator product expansion [1], which is widely used in the case of forward scattering, the non-local operator product expansion [2-5], to which we refer in the present paper, leads to compact expressions for coefficient functions and operators in the non-forward case. As was shown in Ref. [6] the expressions of the local operator product expansions can be obtained by a Taylor expansion of the non-local operator product expansion (cf. also [2, 3]). In lowest order in the coupling constant the expansion contains only quark operators with two external legs. These operators can be decomposed into operators of

different twist. In the present paper we will consider the contribution of the twist-2 operators only and calculate the anomalous dimensions which emerge in the corresponding renormalization group equations.

Our goal is to demonstrate that quite different processes, such as deep inelastic forward scattering, the different channels in deeply virtual Compton scattering, i.e. non-forward scattering at a general kinematics, cf. [7-9] for early studies, and vacuum-meson transitions contain as an essential input the same light-ray operators of twist-2. Therefore the scaling violations of these processes are described by the same anomalous dimensions. The non-perturbative partition 
functions are obtained as the Fourier transforms of the corresponding matrix elements of these operators. With the help of the Fourier representations we derive a new representation for the Compton scattering amplitude in the generalized Bjorken region which extends the representation known for forward scattering. Moreover, the partition functions as the non-perturbative input distributions are related to the Fourier transforms of their matrix elements. Therefore, the evolution equations result directly from the renormalization group equation of the light-ray operators involved. Consequently, all the evolution kernels can be determined from the anomalous dimensions of the corresponding operators. As special cases one obtains the evolution equations for forward scattering and for the case of vacuum-meson transitions. Furthermore the equivalence to other representations in the recent literature is shown, in which different kinematic variables have been used.

In the generalized Bjorken region the distribution amplitudes for the twist-2 contributions depend on two scaling variables unlike the case of forward scattering. One of these parameters is related to the sum, the other to the difference of the initial and final state nucleon momenta. In [10, 11] a solution of the evolution equation for the non-singlet case was obtained. In the literature mostly the one-parametric equations have been discussed so far by imposing further conditions on the momenta. The solution for the singlet case was given in Ref. [12] for the two-variable equations.

The paper is organized as follows. In Section 2 we derive the non-local light cone expansion of the Compton amplitude for non-forward scattering in lowest order. The twist decomposition of the non-local operators is performed in Section 3. In Section 4 the non-forward matrix elements of the non-local operators are calculated and related to the corresponding distribution amplitudes. The double and single-variable distribution amplitudes contributing to the Compton amplitude are discussed in Section 5, where also a derivation of special relations holding in the case of forward scattering off unpolarized and polarized nucleons is presented. In Section 6 a detailed derivation of the anomalous dimensions of the quark and gluon operators contributing at the leading twist level to the non-forward Compton amplitude are given in $O\left(\alpha_{s}\right)$. The evolution kernels are presented in different representations both for unpolarized and polarized scattering. The corresponding evolution equations are discussed in Section 7, both for the case of two and one-variable evolution equations. Here also the kernels for the single-variable equations are given. Special cases of evolution equations into which the general equations transform in a series of kinematic limits are discussed in Section 8, including the cases of forward scattering and vacuum-meson transitions. The solutions of the general two-variable evolution equations are provided in Section 9. Section 10 contains the conclusions. In the Appendices details of the calculation are presented. A brief summary of part of our results has already appeared [13]. In this paper the derivation is presented in detail.

\section{The non-local light-cone expansion in Born approxi- mation}

The Compton amplitude will be evaluated using the non-local light cone expansion (LCE) in terms of bi-local light-ray operators. This expansion is a summed-up version of the local LCE. The latter can be obtained performing a Taylor expansion of the former representation. The rigorous proof of the non-local LCE is rather complicated. Here, following the prescription of Refs. [2, 3, [5, 6], it will be introduced heuristically with some comments on the essential steps.

Firstly, one considers the renormalized $(R)$ time ordered $(T)$ product of two electromagnetic 
currents

$$
R T\left(J_{\mu}(x / 2) J_{\nu}(-x / 2) S\right),
$$

which in perturbative QCD is represented on the Hilbert space of free fields. The electromagnetic current reads

$$
J_{\mu}(x)=\bar{\psi}(x) \gamma_{\mu} \lambda^{\mathrm{em}} \psi(x)
$$

where $\psi(x)$ are the quark fields and

$$
\lambda^{\mathrm{em}}=\frac{1}{2}\left(\lambda_{f}^{3}+\frac{1}{\sqrt{3}} \lambda_{f}^{8}\right) \quad \text { for } \quad S U(3)_{\text {flavor }} ;
$$

$S$ denotes the renormalized S-matrix.

In lowest order in the coupling constant we have $S=1$ and, expressing the $T$-product in terms of normal products, we obtain

$$
\begin{aligned}
R T\left(J_{\mu}(x / 2) J_{\nu}(-x / 2) S\right)= & i: \bar{\psi}(x / 2)\left\{\hat{S}_{\mu \nu \rho \sigma}-i \varepsilon_{\mu \nu \rho \sigma} \gamma_{5}\right\} \gamma^{\sigma}\left(i \partial_{x}^{\rho} D^{c}(x)\right) c^{a} \lambda_{f}^{a} \psi(-x / 2): \\
& -[(x / 2, \mu) \leftrightarrow(-x / 2, \nu)] \\
& + \text { higher order terms }
\end{aligned}
$$

where

$$
\hat{S}_{\mu \nu \rho \sigma} \equiv-S_{\mu \rho \nu \sigma}=g_{\mu \nu} g_{\rho \sigma}-g_{\mu \rho} g_{\nu \sigma}-g_{\mu \sigma} g_{\rho \nu}
$$

and $\varepsilon_{\mu \nu \rho \sigma}$ denotes the Levi-Civita symbol. The $S U(3)_{\text {flavor }}$ vector

$$
c^{a}=\left(\frac{2}{9} \delta^{a 0}+\frac{1}{6} \delta^{a 3}+\frac{\sqrt{3}}{6} \delta^{a 8}\right) e^{2}
$$

with $e$ the electric charge, determines the flavor content and $D^{c}(x)=\left(-i / 4 \pi^{2}\right)\left(x^{2}-i \varepsilon\right)^{-1}$ is the free scalar propagator. Because this expression is restricted to leading order no phase factors occur.

Secondly, since the non-local LCE of composite operators appears as a (multiple) integral representation we hint at it by introducing two auxiliary $\kappa$-integrations [5]

$$
\begin{aligned}
R T\left(J_{\mu}(x / 2) J_{\nu}(-x / 2) S\right)= & \frac{1}{2 \pi^{2}} \frac{c^{a} x^{\rho}}{\left(x^{2}-i \varepsilon\right)^{2}} \int_{-\infty}^{+\infty} d \kappa_{1} \int_{-\infty}^{+\infty} d \kappa_{2} \\
\times & \left\{\frac{i}{2}\left[\bar{\psi}\left(\kappa_{1} x\right) \lambda_{f}^{a} \gamma^{\sigma} \psi\left(\kappa_{2} x\right)-\bar{\psi}\left(\kappa_{2} x\right) \lambda_{f}^{a} \gamma^{\sigma} \psi\left(\kappa_{1} x\right)\right]\left(-\hat{S}_{\mu \nu \rho \sigma}\right) \Delta_{-}\left(\kappa_{1}, \kappa_{2}\right)\right. \\
& \left.+\frac{i}{2}\left[\bar{\psi}\left(\kappa_{1} x\right) \gamma_{5} \lambda_{f}^{a} \gamma^{\sigma} \psi\left(\kappa_{2} x\right)+\bar{\psi}\left(\kappa_{2} x\right) \gamma_{5} \lambda_{f}^{a} \gamma^{\sigma} \psi\left(\kappa_{1} x\right)\right] i \varepsilon_{\mu \nu \rho \sigma} \Delta_{+}\left(\kappa_{1}, \kappa_{2}\right)\right\} \\
& +\ldots
\end{aligned}
$$

where

$$
\Delta_{ \pm}\left(\kappa_{1}, \kappa_{2}\right)=\left[\delta\left(\kappa_{1}-\frac{1}{2}\right) \delta\left(\kappa_{2}+\frac{1}{2}\right) \pm \delta\left(\kappa_{2}-\frac{1}{2}\right) \delta\left(\kappa_{1}+\frac{1}{2}\right)\right]
$$

The next step consists in the projection of the above expressions onto the light-cone by replacing $x \rightarrow \tilde{x}$ with $\tilde{x}^{2}=0$, leaving $x^{2}$ unchanged. The light-like vector

$$
\tilde{x}=x+\frac{\zeta}{\zeta^{2}}\left(\sqrt{(x \zeta)^{2}-x^{2} \zeta^{2}}-(x \zeta)\right)
$$

\footnotetext{
${ }^{1}$ Here, and in the following, the normal product symbols for the relevant operator products will be omitted.
} 
is related to $x$ and a fixed non-null subsidiary four-vector $\zeta$ the dependence of which drops out in leading order expressions. It has to be noted that because the light-cone expansion is considered for the operators and not, as often used, for their matrix elements, a light-like reference vector has necessarily to be introduced in configuration space.'2]

In general, also non-leading contributions related to higher order terms of the $S$-matrix and, in certain gauges, also of the phases factors $U(x / 2,-x / 2)$ have to be taken into account. Then the coefficient functions like $\left(c^{a} / 2 \pi^{2}\right)\left(x^{2}-i \varepsilon\right)^{2} \Delta_{ \pm}\left(\kappa_{1}, \kappa_{2}\right)$ depend non-trivially on $\kappa_{i}$. In this way one arrives at a pre-form of the non-local light-cone expansion being given by a sum over the singular coefficient functions $C_{a}\left(x^{2}, \kappa_{i}\right)$ times the light-ray operators $O^{a}\left(\kappa_{i} \tilde{x}\right)$,

$$
\sum_{a} C_{a}\left(x^{2}, \kappa_{i}\right) \times O^{a}\left(\kappa_{i} \tilde{x}\right)
$$

It should be remarked that even if the perturbative expansion of the renormalized time ordered product of the two currents has been correctly performed, there is no reason to assume that the appearing light-ray operators are the renormalized ones: The expansions resulting from Eq. (2.3) are not yet the true light-cone expansions.

A complete proof which shows that the free field operators appearing in Eq. (2.3) have to be substituted by renormalized light-ray operators is much more involved [2, 3]. Firstly, an operator identity for the product of two composite operators, e.g., the two currents, will be proven which holds on the whole Hilbert space. Then, introducing a new light-cone adapted renormalization procedure $R$ it can be shown that the perturbative functional of the renormalized product of two currents may be split into an asymptotically relevant part - the light-cone expansion up to a definite order in the light-cone singularity - and a well-defined remainder being less singular:

$$
R T\left(J_{\mu}(x / 2) J_{\nu}(-x / 2) S\right) \approx \int_{-1}^{+1} d^{2} \underline{\kappa} C_{\Gamma}\left(x^{2}, \underline{\kappa} ; \mu^{2}\right) R T\left(O^{\Gamma}\left(\kappa_{1} \tilde{x}, \kappa_{2} \tilde{x}\right) S\right)+\text { higher order terms, }
$$

where the free light-ray operators with a specified $\Gamma$-structure are given by

$$
O^{\Gamma}\left(\kappa_{1} \tilde{x}, \kappa_{2} \tilde{x}\right)=\int \frac{d p_{1}}{(2 \pi)^{4}} \frac{d p_{2}}{(2 \pi)^{4}} e^{i \kappa_{1} \tilde{x} p_{1}+i \kappa_{2} \tilde{x} p_{2}}: \bar{\psi}\left(p_{1}\right) \Gamma \psi\left(p_{2}\right):
$$

Here, the coefficient functions and the corresponding light-ray operators appear quite naturally as renormalized ones. In the following, for brevity, we usually do not explicitly indicate that they are renormalized quantities.

Let us point to the fact that, in principle, within this formalism the non-local as well as the usual local light-cone expansion may be obtained (see also [6]). The only difference consists in the following: In the non-local LCE, as an intermediate step to obtain the final representation Eq. (2.4), a Fourier transformation of the renormalized, perturbatively determined coefficient functions $F_{\Gamma}\left(x^{2}, \tilde{x} p_{i}, \mu^{2}\right)$ which multiply the various normal products of free operators in momentum space, like : $\bar{\psi}\left(p_{1}\right) \Gamma \psi\left(p_{2}\right)$ : in Eq. (2.5), has been performed. Primarily, these functions are given as

$$
F_{\Gamma}\left(x^{2}, \tilde{x} p_{i}, \mu^{2}\right)=\int_{-1}^{+1} d^{2} \underline{\kappa} e^{i \kappa_{1} \tilde{x} p_{1}+i \kappa_{2} \tilde{x} p_{2}} C_{\Gamma}\left(x^{2}, \underline{\kappa} ; \mu^{2}\right) .
$$

\footnotetext{
${ }^{2} \tilde{x}$ and its pendant $\tilde{x}^{*}$ with $\tilde{x} \tilde{x}^{*}=1$ correspond to the light-like vectors $n$ and $p$ of dimension mass ${ }^{-1}$ and mass, respectively, usually introduced in deep inelastic scattering according to $P_{\mu}=p_{\mu}+\left(M^{2} / 2\right) n_{\mu}$ [14]. Furthermore, $\tilde{x}$ and $\tilde{x}^{*}$ define in a sense a 'comoving' reference system on the light cone.
} 
In addition, by the $\alpha$-representation it can be proven that they are entire analytic functions with respect to the variables $\tilde{x} p_{i}$ [2, 3] and therefore the range of $\kappa_{i}$ is restricted to

$$
-1 \leq \kappa_{i} \leq+1
$$

On the other hand, in the local $L C E$ one applies a Taylor expansion of $F_{\Gamma}$ with respect to $\tilde{x} p_{i}$ which leads to a (twofold) infinite series over the local operators.

These local and non-local operators are connected by the following relations:

$$
\begin{aligned}
O^{\Gamma}\left(\kappa_{1} \tilde{x}, \kappa_{2} \tilde{x}\right) & =\sum_{n_{1} n_{2}} \frac{\kappa_{1}^{n_{1}}}{n_{1} !} \frac{\kappa_{2}^{n_{2}}}{n_{2} !} O_{n_{1} n_{2}}^{\Gamma}(\tilde{x}), \\
O_{n_{1} n_{2}}^{\Gamma}(\tilde{x}) & =\left.\frac{\partial^{n_{1}}}{\partial \kappa_{1}^{n_{1}}} \frac{\partial^{n_{2}}}{\partial \kappa_{2}^{n_{2}}} O^{\Gamma}\left(\kappa_{1} \tilde{x}, \kappa_{2} \tilde{x}\right)\right|_{\kappa_{1}=\kappa_{2}=0} .
\end{aligned}
$$

With the convention

$$
(\tilde{x} \vec{\partial})^{n} \equiv \tilde{x}^{\mu_{1}} \ldots \tilde{x}^{\mu_{n}} \vec{\partial}_{\mu_{1}} \ldots \vec{\partial}_{\mu_{n}}
$$

and choosing the axial gauge, $\tilde{x}^{\mu} A_{\mu}(x)=0$, we obtain

$$
O_{n_{1} n_{2}}^{\Gamma}(\tilde{x})=\bar{\psi}(0)(\overleftarrow{\partial} \tilde{x})^{n_{1}} \Gamma(\tilde{x} \vec{\partial})^{n_{2}} \psi(0)
$$

Summarizing the foregoing procedure and introducing, for later convenience, the variables

$$
\kappa_{ \pm}=\frac{1}{2}\left(\kappa_{2} \pm \kappa_{1}\right) \quad \text { with } \quad \kappa_{1,2}=\kappa_{+} \mp \kappa_{-},
$$

the general expression will be, taking into account the explicit form of $\hat{S}_{\mu \nu \rho \sigma}$,

$$
\begin{aligned}
& R T\left(J_{\mu}(x / 2) J_{\nu}(-x / 2) S\right) \approx \frac{1}{2} \int D \kappa \\
& \quad \times\left[C_{a}\left(x^{2}, \kappa_{+}, \kappa_{-}, \mu^{2}\right)\left(g_{\mu \nu} O^{a}\left(\kappa_{+} \tilde{x}, \kappa_{-} \tilde{x}, \mu^{2}\right)-\tilde{x}_{\mu} O_{\nu}^{a}\left(\kappa_{+} \tilde{x}, \kappa_{-} \tilde{x}, \mu^{2}\right)-\tilde{x}_{\nu} O_{\mu}^{a}\left(\kappa_{+} \tilde{x}, \kappa_{-} \tilde{x}, \mu^{2}\right)\right)\right. \\
& \left.\quad+i C_{a, 5}\left(x^{2}, \kappa_{+}, \kappa_{-}, \mu^{2}\right) \varepsilon_{\mu \nu}{ }^{\rho \sigma} \tilde{x}_{\rho} O_{5, \sigma}^{a}\left(\kappa_{+} \tilde{x}, \kappa_{-} \tilde{x}, \mu^{2}\right)\right]+ \text { higher order terms, }
\end{aligned}
$$

where the measure

$$
\begin{aligned}
D \kappa & =d \kappa_{1} d \kappa_{2} \theta\left(1-\kappa_{1}\right) \theta\left(1+\kappa_{1}\right) \theta\left(1-\kappa_{2}\right) \theta\left(1+\kappa_{2}\right) \\
& =2 d \kappa_{+} d \kappa_{-} \theta\left(1+\kappa_{+}+\kappa_{-}\right) \theta\left(1+\kappa_{+}-\kappa_{-}\right) \theta\left(1-\kappa_{+}+\kappa_{-}\right) \theta\left(1-\kappa_{+}-\kappa_{-}\right)
\end{aligned}
$$

has been introduced. Here $C_{a(5)}$ are the renormalized coefficient functions which in Born approximation read

$$
\begin{aligned}
C_{a}\left(x^{2}, \kappa_{+}, \kappa_{-}\right) & =\frac{1}{2 \pi^{2}} \frac{c_{a}}{\left(x^{2}-i \varepsilon\right)^{2}} \delta\left(\kappa_{+}\right)\left[\delta\left(\kappa_{-}-\frac{1}{2}\right)-\delta\left(\kappa_{-}+\frac{1}{2}\right)\right], \\
C_{a, 5}\left(x^{2}, \kappa_{+}, \kappa_{-}\right) & =\frac{1}{2 \pi^{2}} \frac{c_{a}}{\left(x^{2}-i \varepsilon\right)^{2}} \delta\left(\kappa_{+}\right)\left[\delta\left(\kappa_{-}-\frac{1}{2}\right)+\delta\left(\kappa_{-}+\frac{1}{2}\right)\right],
\end{aligned}
$$

and $O_{(5) \sigma}^{a}$ are the renormalized (anti)symmetric bi-local LC-operators 9

$$
\begin{aligned}
O_{\sigma}^{a}\left(\kappa_{1}, \kappa_{2}\right) & =\frac{i}{2} R T\left[\bar{\psi}\left(\kappa_{1} \tilde{x}\right) \lambda_{f}^{a} \gamma_{\sigma} U\left(\kappa_{1}, \kappa_{2}\right) \psi\left(\kappa_{2} \tilde{x}\right)-\bar{\psi}\left(\kappa_{2} \tilde{x}\right) \lambda_{f}^{a} \gamma_{\sigma} U\left(\kappa_{2}, \kappa_{1}\right) \psi\left(\kappa_{1} \tilde{x}\right)\right] S, \\
O_{5, \sigma}^{a}\left(\kappa_{1}, \kappa_{2}\right) & =\frac{i}{2} R T\left[\bar{\psi}\left(\kappa_{1} \tilde{x}\right) \lambda_{f}^{a} \gamma_{5} \gamma_{\sigma} U\left(\kappa_{1}, \kappa_{2}\right) \psi\left(\kappa_{2} \tilde{x}\right)+\bar{\psi}\left(\kappa_{2} \tilde{x}\right) \lambda_{f}^{a} \gamma_{5} \gamma_{\sigma} U\left(\kappa_{2}, \kappa_{1}\right) \psi\left(\kappa_{1} \tilde{x}\right)\right] S,
\end{aligned}
$$

\footnotetext{
${ }^{3}$ In the following $\left(\kappa_{1}, \kappa_{2}\right)$ and $\left(\kappa_{+}, \kappa_{-}\right)$are chosen interchangeably, i.e. $O\left(\kappa_{+} \tilde{x}, \kappa_{-} \tilde{x}\right)$ is written instead of the more lengthly expression $O\left(\left(\kappa_{+}-\kappa_{-}\right) \tilde{x},\left(\kappa_{+}+\kappa_{-}\right) \tilde{x}\right)$. Furthermore, very often $\tilde{x}$ and the renormalization scale $\mu^{2}$ will be suppressed in the arguments of the operators and related quantities for brevity.
} 
respectively, with the phase factors

$$
U\left(\kappa_{1}, \kappa_{2}\right) \equiv U\left(\kappa_{1} \tilde{x}, \kappa_{2} \tilde{x}\right)=\mathcal{P} \exp \left\{-i g \int_{\kappa_{2}}^{\kappa_{1}} d \tau \tilde{x}^{\mu} A_{\mu}(\tau \tilde{x})\right\}
$$

where $\mathcal{P}$ denotes the path ordering, $g$ is the strong coupling constant and $A_{\mu}=A_{\mu}^{a} t^{a}$ is the gluon field with $t^{a}$ being the generators of $S U(3)_{\text {color }}$ in the fundamental representation spanned by the quark fields. In the explicit expressions we have indicated by $R T[O] S$ that these operators are renormalized.

The general expression Eq. (2.12) contains a flavor singlet as well as the flavor non-singlet (NS) parts. In the case of flavor non-singlet operators, i.e., for $\lambda_{f}^{a} \neq 1$, the consideration of the quark operators Eqs. (2.16) and (2.17) is sufficient. However, the singlet operators $\left(\lambda_{f}^{0} \equiv 1\right)$, in the following denoted by $O^{q}$, mix with certain gluon operators which will be denoted by $O^{G}$. In addition, if we would consider the Compton amplitude beyond leading order also the gluon operators would appear in the LCE.

The gluon operators related to the quark operators Eqs. (2.16) and (2.17) read

$$
\begin{aligned}
O_{\mu \nu}^{G}\left(\kappa_{1}, \kappa_{2}\right) & =\frac{1}{2} R T\left[F_{\mu}^{a \lambda}\left(\kappa_{1} \tilde{x}\right) U^{a b}\left(\kappa_{1}, \kappa_{2}\right) F_{\nu \lambda}^{b}\left(\kappa_{2} \tilde{x}\right)+F_{\mu}^{a \lambda}\left(\kappa_{2} \tilde{x}\right) U^{a b}\left(\kappa_{2}, \kappa_{1}\right) F_{\nu \lambda}^{b}\left(\kappa_{2} \tilde{x}\right)\right] S \\
O_{5 \mu \nu}^{G}\left(\kappa_{1}, \kappa_{2}\right) & =\frac{1}{2} R T\left[F_{\mu}^{a \lambda}\left(\kappa_{1} \tilde{x}\right) U^{a b}\left(\kappa_{1}, \kappa_{2}\right) \widetilde{F}_{\nu \lambda}^{b}\left(\kappa_{2} \tilde{x}\right)-F_{\mu}^{a \lambda}\left(\kappa_{2} \tilde{x}\right) U^{a b}\left(\kappa_{2}, \kappa_{1}\right) \widetilde{F}_{\nu \lambda}^{b}\left(\kappa_{1} \tilde{x}\right)\right] S,
\end{aligned}
$$

where,

$$
F_{\mu \nu} \equiv F_{\mu \nu}^{a} t^{a} \quad \text { and } \quad \widetilde{F}_{\mu \nu}=\frac{1}{2} \varepsilon_{\mu \nu \rho \sigma} F^{\rho \sigma}
$$

is the gluon field strength and the dual field strength, respectively. $U^{a b}\left(\kappa_{1}, \kappa_{2}\right)$ is the phase factor (2.18) in the adjoint representation. To obtain exact correspondence to the above quark operators the gluon operators have to be multiplied by $\frac{1}{2}\left(g_{\sigma}{ }^{\mu} \tilde{x}^{\nu}+g_{\sigma}{ }^{\nu} \tilde{x}^{\mu}\right)$.

\section{Twist decomposition of non-local operators}

The non-local operators Eqs. (2.16, 2.17, 2.19, 2.20) contain contributions of different twist. Here we use the notion of twist in its original definition 15] as geometric twist, i.e. canonical dimension minus spin of the corresponding operator. Let us now extract the leading twist parts, which are relevant for the discussion of virtual Compton scattering. This concerns the contributions of twist two and three, the latter being especially relevant for the antisymmetric part of the scattering amplitude which concerns the polarized scattering.

We consider first the quark operators $O_{\sigma}^{a}$ and $O_{5, \sigma}^{a}$, which contain contributions of twist-2, 3 and 4:

$$
\begin{aligned}
O_{\sigma}^{a} & =O_{\sigma}^{a, \text { twist } 2}+O_{\sigma}^{a, \text { twist } 3}+O_{\sigma}^{a, \text { twist } 4} \\
O_{5, \sigma}^{a} & =O_{5, \sigma}^{a, \text { twist } 2}+O_{5, \sigma}^{a, \text { twist } 3}+O_{5, \sigma}^{a, \text { twist } 4}
\end{aligned}
$$

In principle also higher twist contributions appear, but they vanish on the light-cone since they are proportional to nonvanishing integer powers of $\tilde{x}^{2}$.

To obtain the bi-local operators (3.1) of definite twist we consider the centered operators

$$
\bar{\psi}(-\kappa x) \lambda_{f}^{a} \gamma_{\sigma} U(-\kappa x, \kappa x) \psi(\kappa x)
$$


with arbitrary values of $x \neq \tilde{x}$, i.e., we consider the operators as not restricted to the light-cone. They are represented through the Taylor expansion Eq. (2.8) by a series of local tensor operators of order $n=n_{1}+n_{2}$ which, in the axial gauge, may be written as

$$
\sum_{n} \frac{\kappa^{n}}{n !} x^{\mu_{1}} \ldots x^{\mu_{n}} \bar{\psi}(0) \stackrel{\leftrightarrow}{\partial}_{\mu_{1}} \ldots \stackrel{\leftrightarrow}{\partial}_{\mu_{n}} \lambda_{f}^{a} \gamma_{\sigma} \psi(0)
$$

Here we used the abbreviation $\stackrel{\leftrightarrow}{\partial} \equiv \vec{\partial}-\overleftarrow{\partial}$. By purely group theoretical methods these local operators are decomposed into traceless tensor operators of definite dimension and spin carrying an irreducible representation of the Lorentz group. The symmetry behavior of these tensors is characterized by a few special classes of Young tableaux'. As is well known, the twist-2 terms correspond to the total symmetric representation. Finally, the local operators of definite twist are resummed to give non-local harmonic tensor operators of definite twist.

Technically, these non-local harmonic tensor operators are obtained from the initial ones by first projecting onto traceless operators and then applying appropriate differential operators related to the corresponding symmetry class. Finally, the resulting expressions are projected onto the light-ray $\tilde{x}$. In principle this method can also be applied for arbitrary values of $\kappa_{i}$.

This approach has been worked out in Refs. [16, 17] for various light-ray operators. Here, we refer only to those representations which are relevant for the subsequent considerations. First the projections onto the traceless operators are defined. For the non-local scalar and vector quark operators Eqs. (2.16) and (2.17), respectively, they are given by

$$
\begin{aligned}
O^{q, \text { traceless }}(-\kappa x, \kappa x)= & \frac{i}{2}[\bar{\psi}(-\kappa x)(x \gamma) \psi(\kappa x)-\bar{\psi}(\kappa x)(x \gamma) \psi(-\kappa x)] \\
+ & \sum_{k=1}^{\infty} \int_{0}^{1} d t\left(\frac{1-t}{t}\right)^{k-1}\left(\frac{-x^{2}}{4}\right)^{k} \frac{\square^{k}}{k !(k-1) !} \\
& \times \frac{i}{2}[\bar{\psi}(-\kappa t x)(x \gamma) \psi(\kappa t x)-\bar{\psi}(\kappa t x)(x \gamma) \psi(-\kappa t x)], \\
O_{\sigma}^{q, \text { traceless }}(-\kappa x, \kappa x)= & \frac{i}{2}\left[\bar{\psi}(-\kappa x) \gamma_{\sigma} \psi(\kappa x)-\bar{\psi}(\kappa x) \gamma_{\sigma} \psi(-\kappa x)\right] \\
+ & \sum_{k=1}^{\infty} \int_{0}^{1} \frac{d t}{t}\left(\frac{1-t}{t}\right)^{k-1}\left(\frac{-x^{2}}{4}\right)^{k} \frac{\square^{k}}{k !(k-1) !} \\
& \times \frac{i}{2}\left[\bar{\psi}(-\kappa t x) \gamma_{\sigma} \psi(\kappa t x)-\bar{\psi}(\kappa t x) \gamma_{\sigma} \psi(-\kappa t x)\right] \\
+ & \frac{1}{2}\left[\left(x_{\sigma} x_{\rho} \square+x^{2} \partial_{\sigma} \partial_{\rho}\right)-2 x_{\sigma} \partial_{\rho}(x \partial)\right] \\
& \times \sum_{k=0}^{\infty} \int_{0}^{1} d \tau \int_{0}^{1} d t\left(\frac{1-t}{t}\right)^{k}\left(\frac{-x^{2}}{4}\right)^{k} \frac{\square^{k}}{k ! k !} \\
& \times \frac{i}{2}\left[\bar{\psi}(-\kappa \tau t x) \gamma^{\rho} \psi(\kappa \tau t x)-\bar{\psi}(\kappa \tau t x) \gamma^{\rho} \psi(-\kappa \tau t x)\right] .
\end{aligned}
$$

Here the phase factors and the generators $\lambda_{f}^{a}$ of the flavor group have been suppressed and the operators generically are indicated by $q$. The sum over terms containing the d'Alembertian are the subtractions of the traces. The representation for $O^{q \text {, traceless }}$, Eq. (3.4), has been obtained already earlier in Ref. [18]. Taking the limit $x \rightarrow \tilde{x}$ in Eq. (3.4) the additional terms vanish because of $\tilde{x}^{2}=0$. Therefore the operator $\tilde{x}^{\sigma} O_{\sigma}^{q}(-\kappa \tilde{x}, \kappa \tilde{x})$ is of twist two:

$$
O^{q, \text { twist2 }}(-\kappa \tilde{x}, \kappa \tilde{x})=O^{q, \text { traceless }}(-\kappa \tilde{x}, \kappa \tilde{x}) \equiv O^{q}(-\kappa \tilde{x}, \kappa \tilde{x}),
$$


Because of their tracelessness the above operators fulfill the following relations

$$
\square O^{q, \text { traceless }}(-\kappa x, \kappa x)=0, \quad \partial^{\sigma} O_{\sigma}^{q, \text { traceless }}(-\kappa x, \kappa x)=0, \quad \square O_{\sigma}^{q, \text { traceless }}(-\kappa x, \kappa x)=0 .
$$

which proves that they are harmonic scalar and vector operators, respectively. The differential operators, to be applied to $O^{q \text {, traceless }}$ and $O_{\rho}^{q \text {, traceless }}$ in order to generate the correct symmetry behavior, are $\partial_{\sigma}$ for twist -2 and $\left(g_{\sigma \rho}(x \partial)-x_{\sigma} \partial_{\rho}\right)$ for twist-3, respectively. Note that taking these derivatives before carrying out the light-cone limit leads to non-trivial contributions which result from the trace terms.

The explicit computations straightforwardly lead to the following expressions for the twist-2, twist-3 and twist-4 light-ray vector operators [16, 17]:

$$
\begin{aligned}
O_{\sigma}^{q, \text { twist } 2}(-\kappa \tilde{x}, \kappa \tilde{x}) & =\left.\int_{0}^{1} d \tau \partial_{\sigma} O_{\text {traceless }}^{q}(-\kappa \tau x, \kappa \tau x)\right|_{x \rightarrow \tilde{x}} \\
& =\left.\int_{0}^{1} d \tau\left[\partial_{\sigma}+\frac{1}{2}(\ln \tau) x_{\sigma} \square\right] O^{q}(-\kappa \tau x, \kappa \tau x)\right|_{x=\tilde{x}}, \\
O_{\sigma}^{q, \text { twist } 3}(-\kappa \tilde{x}, \kappa \tilde{x}) & =\left.\int_{0}^{1} d \tau\left(g_{\sigma \rho} x \partial-x_{\sigma} \partial_{\rho}\right) O_{\text {traceless }}^{q \rho}(-\kappa \tau x, \kappa \tau x)\right|_{x=\tilde{x}}, \\
& =\int_{0}^{1} d \tau\left[\left(g_{\sigma \rho} x \partial-x_{\sigma} \partial_{\rho}\right)\right. \\
O_{\sigma}^{q, \text { twist } 4}(-\kappa \tilde{x}, \kappa \tilde{x}) & =\left.x_{\sigma} \int_{0}^{1} d \tau\left[(1+\ln \tau) \partial_{\rho}+\frac{1}{2}(\ln \tau) x_{\rho}^{\square}\right] O^{q \rho}(-\kappa \tau x, \kappa \tau x)\right|_{x=\tilde{x}},
\end{aligned}
$$

where the last expression is obtained using Eq. (3.1). Note that the operators appearing at the r.h.s. of Eqs. (3.8 - 3.10) are to be taken with the phase factors before carrying out the derivatives.

Important properties of these operators are

$$
\tilde{x}^{\sigma} O_{\sigma}^{q, \text { twist2 }}=O^{q, \text { twist2 }}, \quad \tilde{x}^{\sigma} O_{\sigma}^{q, \text { twist3 }}=0, \quad \tilde{x}^{\sigma} O_{\sigma}^{q, \text { twist4 }}=0 .
$$

Analogous representations hold for $O_{5, \sigma}^{q, \text { twist2 }}, O_{5, \sigma}^{q \text {, twist3 }}$ and $O_{5, \sigma}^{q, \text { twist4 }}$, which have similar properties. Note that the operators (3.8-3.10) include the trace terms which are proportional to $\tilde{x}_{\sigma}$. In fact they are again summed-up local operators fulfilling Eq. (2.8).

Let us now consider the gluon operators Eqs. (2.19) and (2.20). Again they contain a twist-2, twist- 3 and twist-4 part, related to the traceless symmetric, the antisymmetric and the trace part of the tensor $O_{(5) \mu \nu}^{G}$, respectively. Since we are concerned with the scalar and vector gluon operators,

$$
\tilde{x}^{\mu} \tilde{x}^{\nu} O_{(5) \mu \nu}^{G} \quad \text { and } \quad \frac{1}{2}\left(g_{\sigma}^{\mu} \tilde{x}^{\nu}+g_{\sigma}^{\nu} \tilde{x}^{\mu}\right) O_{(5) \mu \nu}^{G}
$$

only the symmetric twist-2 part will be considered here. In the same manner as in the case of quark operators we first define the projection onto the centered traceless operator. It is given by

$$
\begin{aligned}
O^{G, \text { traceless }}(-\kappa x, \kappa x)= & \frac{1}{2} x_{\mu} x_{\nu}\left[F_{\mu}^{a \lambda}(-\kappa x) F_{\nu \lambda}^{a}(\kappa x)+F_{\mu}^{a \lambda}(\kappa x) F_{\nu \lambda}^{a}(-\kappa x)\right] \\
+ & \sum_{k=1}^{\infty} \int_{0}^{1} d t t\left(\frac{1-t}{t}\right)^{k-1}\left(\frac{-x^{2}}{4}\right)^{k} \frac{\square^{k}}{k !(k-1) !} \\
& \times \frac{1}{2} x_{\mu} x_{\nu}\left[F_{\mu}^{a \lambda}(-\kappa t x) F_{\nu \lambda}^{a}(\kappa t x)+F_{\mu}^{a \lambda}(\kappa t x) F_{\nu \lambda}^{a}(-\kappa t x)\right],
\end{aligned}
$$


where again the phase factors have been suppressed. It fulfills the relation

$$
\square O^{G, \text { traceless }}(-\kappa x, \kappa x)=0 .
$$

In comparison with Eq. (3.4) only an additional factor of $t$ appears under the integral. This results from the fact that the operator (2.19) relative to (2.16) contains one more factor of $x$ not being accompanied by the parameter $\kappa$. The scalar and vector twist-2 gluon light-ray operators are now given by

$$
\begin{aligned}
O^{G, \text { twist2 }}(-\kappa \tilde{x}, \kappa \tilde{x}) & =O^{G, \text { traceless }}(-\kappa \tilde{x}, \kappa \tilde{x}) \equiv O^{G}(-\kappa \tilde{x}, \kappa \tilde{x}) \\
O_{\mu}^{G, \text { twist2 }}(-\kappa \tilde{x}, \kappa \tilde{x}) & =\left.\int_{0}^{1} d \tau \tau \partial_{\mu} O^{G, \text { traceless }}(-\kappa \tau x, \kappa \tau x)\right|_{x=\tilde{x}} \\
& =\left.\int_{0}^{1} d \tau \tau\left[\partial_{\mu}+\frac{1}{2}(\ln \tau) x_{\mu} \square\right] O^{G}(-\kappa \tau x, \kappa \tau x)\right|_{x=\tilde{x}} .
\end{aligned}
$$

Here we gave for simplicity the expressions for operators with the special arguments $\kappa_{1}=$ $-\kappa, \kappa_{2}=\kappa$. However, any of the Eqs. 3.4 - 3.15) remains true if general arguments are chosen. As an example the singlet vector operators of twist-2 read

$$
O_{\mu}^{A, \text { twist } 2}\left(\kappa_{1} \tilde{x}, \kappa_{2} \tilde{x}\right)=\left.\int_{0}^{1} d \tau \tau^{d_{A}-1}\left[\partial_{\mu}+\frac{1}{2}(\ln \tau) x_{\mu} \square\right] O^{A}\left(\kappa_{1} \tau x, \kappa_{2} \tau x\right)\right|_{x=\tilde{x}}
$$

where $A=(q, G)$ and $d_{q}=1, d_{G}=2$. In addition, let us emphasize that in the various integrals defining operators of definite twist the variables $\kappa_{i}$ have to be scaled but not the coordinates $x$ of the corresponding (original) operators.

The general form of harmonic operators of definite twist is necessary for an investigation of their matrix elements whereas the special centered form is sufficient to determine their anomalous dimensions, as will be shown in Sections 4 and 6, respectively. Inserting the result obtained for the twist-2 terms, Eq. (3.8), into Eq. (2.12) we obtain an asymptotic representation of the Compton scattering amplitude Eq. (1.2) which is valid in the generalized Bjorken region, as will be shown in Section 5 in detail. Concerning the renormalization of the twist-2 singlet operators and the evolution equations of the corresponding distribution amplitudes also the representations Eq. (3.14) and (3.15) necessarily come into play.

\section{Matrix elements of non-local operators}

The Compton scattering amplitude is obtained by taking the matrix elements of the operator, Eq. (2.12), between the hadron states $\left|p_{i}, S_{i}\right\rangle$. Thereby the matrix elements of the light-ray operators Eqs. (2.16) and (2.17) appear as non-perturbative inputs. These quantities are represented by partition functions in the forward case and distribution amplitudes in the case of non-forward scattering. Here, and for the description of the evolution equations below, the general form of the operators $O^{\Gamma}\left(\kappa_{1} \tilde{x}, \kappa_{2} \tilde{x}\right)$ has to be taken into account. It will be shown that their Fourier transforms with respect to $\kappa_{-} \tilde{x} p_{+}$and $\kappa_{-} \tilde{x} p_{-}$define the above mentioned distribution amplitudes. For the following considerations it is also useful, as it was in the twist decomposition, to study the non-local operators temporarily to arbitrary values of $x$ and to perform the light cone limit only afterwards.

In the following we consider the contributions of twist-2 only. In the case of purely electromagnetic interactions the matrix elements at lowest order in the coupling constant, using the 
equations of motion, can be represented by two basic hadronic matrix elements, including also the spin dependence:

$$
\begin{aligned}
& \mathcal{Q}^{D}\left(x ; p_{2}, p_{1}\right) \equiv x^{\mu} \mathcal{Q}_{\mu}^{D}\left(p_{2}, p_{1}\right)=x^{\mu} \bar{u}\left(p_{2}, S_{2}\right) \gamma_{\mu} u\left(p_{1}, S_{1}\right) \\
& \mathcal{Q}^{P}\left(x ; p_{2}, p_{1}\right) \equiv x^{\mu} \mathcal{Q}_{\mu}^{P}\left(p_{2}, p_{1}\right)=x^{\mu} \bar{u}\left(p_{2}, S_{2}\right) \frac{1}{M} \sigma_{\mu \nu} p_{-}^{\nu} u\left(p_{1}, S_{1}\right)
\end{aligned}
$$

with

$$
\sigma_{\mu \nu}=\frac{i}{2}\left[\gamma_{\mu}, \gamma_{\nu}\right]
$$

$M$ is the nucleon mass, and $u(p, S)$ denotes a free hadronic Dirac spinor. Here the indices $D$ and $P$ refer to the Dirac and Pauli structure, respectively.

Let us first consider the matrix elements of the scalar twist-2 quark operator. Because of translation invariance,

$$
\begin{aligned}
\left\langle p_{2}\left|O^{\Gamma}\left(\kappa_{1} x, \kappa_{2} x\right)\right| p_{1}\right\rangle & \equiv\left\langle p_{2}\left|O^{\Gamma}\left(\left(\kappa_{+}-\kappa_{-}\right) x,\left(\kappa_{+}+\kappa_{-}\right) x\right)\right| p_{1}\right\rangle \\
& =e^{i \kappa_{+} x p_{-}}\left\langle p_{2}\left|O^{\Gamma}\left(-\kappa_{-} x, \kappa_{-} x\right)\right| p_{1}\right\rangle
\end{aligned}
$$

it is completely sufficient to consider the matrix elements of the centered operators:

$$
\left\langle p_{2}\left|O^{q, \text { twist2 }}\left(-\kappa_{-} x, \kappa_{-} x\right)\right| p_{1}\right\rangle=i \tilde{f}_{J}^{q}\left(\kappa_{-} x p_{+}, \kappa_{-} x p_{-}, \kappa_{-}^{2} x^{2}, p_{1} p_{2}, \mu^{2}\right) \mathcal{Q}^{J}\left(x ; p_{2}, p_{1}\right),
$$

where the summation is taken over $J=(D, P)$. If considered independently these functions will be denoted by $f_{D} \equiv g$ and $f_{P} \equiv h$. The generalization to arbitrary values of $\kappa_{i}$ is trivial due to translation invariance, Eq.(4.3), through which a factor $\exp \left\{i \kappa_{+} \tilde{x} p_{-}\right\}$occurs on both sides of Eq. (4.4). The projection onto the light-cone immediately gives

$$
\left\langle p_{2}\left|O^{q, \operatorname{twist} 2}\left(-\kappa_{-} \tilde{x}, \kappa_{-} \tilde{x}\right)\right| p_{1}\right\rangle=i \tilde{f}_{J}^{q}\left(\kappa_{-} \tilde{x} p_{+}, \kappa_{-} \tilde{x} p_{-}, p_{1} p_{2}, \mu^{2}\right) \mathcal{Q}^{J}\left(\tilde{x} ; p_{2}, p_{1}\right) .
$$

The twist-2 vector operators, because of the additional derivative in Eq. (3.8), do not have such a simple representation. Using that relation between the vector operator and the scalar operator for general arguments we obtain

$$
\begin{aligned}
\left\langle p_{2}\left|O_{\mu}^{q, \text { twist } 2}\left(\kappa_{1} \tilde{x}, \kappa_{2} \tilde{x}\right)\right| p_{1}\right\rangle=\left.\int_{0}^{1} d \lambda \partial_{\mu}^{x}\left\langle p_{2}\left|O^{q, \text { twist2 }}\left(\kappa_{1} \lambda x, \kappa_{2} \lambda x\right)\right| p_{1}\right\rangle\right|_{x=\tilde{x}} \\
\quad=\left.i \int_{0}^{1} d \lambda \partial_{\mu}^{x}\left(\tilde{f}_{J}^{q}\left(\kappa_{-} \lambda x p_{+}, \kappa_{-} \lambda x p_{-}, \kappa_{-}^{2} \lambda^{2} x^{2}, p_{1} p_{2}, \mu^{2}\right) \mathcal{Q}^{J}\left(x ; p_{2}, p_{1}\right) e^{i \kappa_{+} \lambda x p_{-}}\right)\right|_{x=\tilde{x}}
\end{aligned}
$$

Let us now perform the Fourier transformations of $\tilde{f}_{J}$ with respect to $\kappa_{-} \lambda x p_{ \pm} \equiv \kappa x p_{ \pm}$,

$$
\tilde{f}\left(\kappa x p_{+}, \kappa x p_{-}, \kappa^{2} x^{2}\right)=\int D z e^{-i \kappa x\left(p_{i} z_{i}\right)} f\left(z_{+}, z_{-}, \kappa^{2} x^{2}\right)
$$

where

$$
\left(p_{i} z_{i}\right) \equiv p(z)=p_{+} z_{+}+p_{-} z_{-} \quad \text { with } \quad z_{ \pm}=\frac{1}{2}\left(z_{2} \pm z_{1}\right)
$$

defines a scalar product in the space of two-vectors labeled either by $(1,2)$ or $(+,-)$. Also these functions, if projected onto the light-cone, are entire analytic functions with respect to $\kappa_{-} \tilde{x} p_{i}$ and therefore the support of their Fourier transforms is restricted to the range

$$
-1 \leq z_{i} \leq 1
$$


Thereby, we assumed that the kinematic decomposition of the matrix elements does not introduce additional kinematic singularities. Therefore, the integration measure in Eq. (4.7), analogous to Eq. (2.13), is given by

$$
\begin{aligned}
D z & =\frac{1}{2} d z_{1} d z_{2} \theta\left(1-z_{1}\right) \theta\left(1+z_{1}\right) \theta\left(1-z_{2}\right) \theta\left(1+z_{2}\right) \\
& =d z_{+} d z_{-} \theta\left(1+z_{+}+z_{-}\right) \theta\left(1+z_{+}-z_{-}\right) \theta\left(1-z_{+}+z_{-}\right) \theta\left(1-z_{+}-z_{-}\right)
\end{aligned}
$$

The $\lambda$-integration in Eq. (4.6) can be performed leading to

$$
\begin{aligned}
\left\langle p_{2}\left|O_{\mu}^{q, \text { twist } 2}\left(\kappa_{1} \tilde{x}, \kappa_{2} \tilde{x}\right)\right| p_{1}\right\rangle=i \int D z e^{-i \kappa_{-}\left(\tilde{x} p_{i}\right) z_{i}} & \\
\times & \left\{F_{J}^{q}\left(z_{+}, z_{-}, \kappa_{+} \tilde{x} p_{-}, \kappa_{-}^{2} x^{2}\right)\left(\mathcal{Q}_{\mu}^{J}\left(p_{2}, p_{1}\right)-i \kappa_{-} p_{\mu}(z) \mathcal{Q}^{J}\left(x ; p_{2}, p_{1}\right)\right)\right. \\
& \left.+\left(i \kappa_{+} p_{-}^{\mu} \partial_{i \kappa_{+} x p_{-}}+2 x_{\mu} \kappa_{-}^{2} \partial_{\kappa_{-}^{2} x^{2}}\right) F_{J}^{q}\left(z_{+}, z_{-}, \kappa_{+} x p_{-}, \kappa_{-}^{2} x^{2}\right) \mathcal{Q}^{J}\left(x ; p_{2}, p_{1}\right)\right\}\left.\right|_{x=\tilde{x}} .
\end{aligned}
$$

Here, the following functions $F_{J}^{q}\left(z_{+}, z_{-}, \kappa_{+} x p_{-}, \kappa_{-}^{2} x^{2}\right)$ are introduced:

$$
\begin{aligned}
F\left(z_{+}, z_{-}, \kappa_{+} x p_{-}, \kappa_{-}^{2} x^{2}\right) & =\int_{0}^{1} \frac{d \lambda}{\lambda^{2}} f\left(\frac{z_{+}}{\lambda}, \frac{z_{-}}{\lambda}, \kappa_{-}^{2} \lambda^{2} x^{2}\right) \Theta\left(\lambda-\left|z_{+}\right|\right) \Theta\left(\lambda-\left|z_{-}\right|\right) e^{i \kappa_{+} \lambda x p_{-}}, \text {(4.11) } \\
\stackrel{o}{F}\left(z_{+}, z_{-}, \kappa_{+} x p_{-}, \kappa_{-}^{2} x^{2}\right) & \equiv\left(\partial_{i \kappa_{+} x p_{-}} F\right)\left(z_{+}, z_{-}, \kappa_{+} x p_{-}, \kappa_{-}^{2} x^{2}\right) \\
& =\int_{0}^{1} \frac{d \lambda}{\lambda} f\left(\frac{z_{+}}{\lambda}, \frac{z_{-}}{\lambda}, \kappa_{-}^{2} \lambda^{2} x^{2}\right) \Theta\left(\lambda-\left|z_{+}\right|\right) \Theta\left(\lambda-\left|z_{-}\right|\right) e^{i \kappa_{+} \lambda x p_{-}} \\
F^{\prime}\left(z_{+}, z_{-}, \kappa_{+} x p_{-}, \kappa_{-}^{2} x^{2}\right) & \equiv\left(\partial_{\kappa_{-}^{2} x^{2}} F\right)\left(z_{+}, z_{-}, \kappa_{+} x p_{-}, \kappa_{-}^{2} x^{2}\right) \\
& =\int_{0}^{1} d \lambda \partial_{\kappa_{-}^{2} \lambda^{2} x^{2}} f\left(\frac{z_{+}}{\lambda}, \frac{z_{-}}{\lambda}, \kappa_{-}^{2} \lambda^{2} x^{2}\right) \Theta\left(\lambda-\left|z_{+}\right|\right) \Theta\left(\lambda-\left|z_{-}\right|\right) e^{i \kappa_{+} \lambda x p_{-}} .
\end{aligned}
$$

As will be shown in Section 5 one consequence of the representation (4.11) are the integral relations in polarized deeply inelastic scattering in the forward case [19] and [20], as well as the Callan-Gross relation [21. For $p_{-}=0$ one obtains:

$$
F(z)=\int_{0}^{1} \frac{d \lambda}{\lambda} f\left(\frac{z}{\lambda}\right) \Theta(\lambda-z)=\int_{z}^{1} \frac{d z}{z} f(z) .
$$

Thereby the derivation $f^{\prime}\left(z_{+}, z_{-}, \kappa_{-}^{2} x^{2}\right) \equiv\left(\partial_{\kappa_{-}^{2} x^{2}} f\right)\left(z_{+}, z_{-}, \kappa_{-}^{2} x^{2}\right)$ was introduced in Eq. (4.13). Furthermore, a third Fourier transformation can be performed either with respect to $\kappa_{-}^{2} x^{2}$ [11] or, as will be used here, with respect to $\kappa_{-} x$, which allows to study the $\left(1 / Q^{2}\right)$-dependence of the distribution amplitudes

$$
\tilde{f}\left(\kappa_{-} x p_{+}, \kappa_{-} x p_{-}, \kappa_{-}^{2} x^{2}\right)=\int D z \int d^{4} q e^{-i \kappa_{-}\left(x p_{i}\right) z_{i}} e^{-i \kappa_{-} q x} f\left(z_{+}, z_{-}, q^{2}\right) .
$$

In the limiting case of forward scattering we have:

$$
\mathcal{Q}_{D}(x ; p, p) \equiv \bar{u}(p) x^{\mu} \gamma_{\mu} u(p)=2 x p \quad \text { and }\left.\quad \mathcal{Q}_{P}(x ; p, p) \equiv \bar{u}\left(p_{2}\right) \frac{1}{M} x^{\mu} \sigma_{\mu \nu} p_{-}^{\nu} u\left(p_{1}\right)\right|_{p_{2} \rightarrow p_{1}}=0
$$

and one obtains $\left(g \equiv f_{D}\right)$

$$
\begin{aligned}
\left\langle p\left|O^{q, \text { twist2}}\left(-\kappa_{-} \tilde{x}, \kappa_{-} \tilde{x}\right)\right| p\right\rangle & =i 2(\tilde{x} p) \tilde{g}^{q}\left(2 \kappa_{-} \tilde{x} p\right) \\
& =i 2(\tilde{x} p) \int_{-1}^{1} d z_{+} e^{-2 i \kappa_{-}(\tilde{x} p) z_{+}} \int_{-1+\left|z_{+}\right|}^{+1-\left|z_{+}\right|} d z_{-} g^{q}\left(z_{+}, z_{-}\right)
\end{aligned}
$$


Analogous decompositions and representations are valid for the matrix elements of the pseudoscalar and pseudo-vector twist-2 quark operators. The corresponding partition functions are denoted by $f_{J, 5}^{q}=\left(g_{5}^{q}, h_{5}^{q}\right)$ and $F_{J, 5}^{q}=\left(G_{5}^{q}, H_{5}^{q}\right)$, respectively, and the Dirac and Pauli structures are to be replaced by $\mathcal{Q}_{5}^{D}, \mathcal{Q}_{5}^{P}$ instead of Eqs. (4.1, 4.2), substituting $\gamma_{\mu} \rightarrow \gamma_{5} \gamma_{\mu}$ and $\sigma_{\mu \nu} \rightarrow \gamma_{5} \sigma_{\mu \nu}$, respectively.

Let us now extend our consideration to the twist-2 scalar and vector gluon operators, Eqs. (3.14) and (3.15). Completely analogous to Eq. (4.4) the matrix elements of Eq. (3.14) are written as

$$
\left\langle p_{2}\left|O^{G, \text { twist2 }}\left(-\kappa_{-} x, \kappa_{-} x\right)\right| p_{1}\right\rangle=i\left(i x p_{+}\right) \tilde{f}_{J}^{G(1)}\left(\kappa_{-} x p_{+}, \kappa_{-} x p_{-}, \kappa_{-}^{2} x^{2}, p_{1} p_{2}, \mu^{2}\right) \mathcal{Q}^{J}\left(x ; p_{2}, p_{1}\right) .
$$

The additional factor $i x p_{+}$has been introduced in order to compensate for the different $\kappa$-scale dimensions, $d_{q}=1$ and $d_{G}=2$, of the quark and gluon operators, respectively, cf. also Section 6.1 .

The matrix elements of the twist-2 gluonic vector operators, Eq. (3.15), are obtained in the same manner as for the corresponding quark operators:

$$
\begin{aligned}
& \left\langle p_{2}\left|O_{\mu}^{G, \text { twist2 }}\left(\kappa_{1} \tilde{x}, \kappa_{2} \tilde{x}\right)\right| p_{1}\right\rangle=i \int D z e^{-i \kappa_{-}\left(x p_{i}\right) z_{i}} \\
& \times\left\{( i x p _ { + } ) \left[F_{J}^{G(1)}\left(z_{+}, z_{-}, \kappa_{+} x p_{-}, \kappa_{-}^{2} x^{2}\right)\left(\mathcal{Q}_{\mu}^{J}\left(p_{2}, p_{1}\right)-i \kappa_{-} p_{\mu}(z) \mathcal{Q}^{J}\left(x ; p_{2}, p_{1}\right)\right)\right.\right. \\
& \left.+\left(i \kappa_{+} p_{-}^{\mu} \partial_{i \kappa_{+} x p_{-}}+2 x_{\mu} \kappa_{-}^{2} \partial_{\kappa_{-}^{2} x^{2}}\right) F_{J}^{G(1)}\left(z_{+}, z_{-}, \kappa_{+} x p_{-}, \kappa_{-}^{2} x^{2}\right) \mathcal{Q}^{J}\left(x ; p_{2}, p_{1}\right)\right] \\
& \left.+i p_{+}^{\mu} F_{J}^{G(1)}\left(z_{+}, z_{-}, \kappa_{+} x p_{-}, \kappa_{-}^{2} x^{2}\right) \mathcal{Q}^{J}\left(x ; p_{2}, p_{1}\right)\right\}\left.\right|_{x=\tilde{x}} .
\end{aligned}
$$

We remark that in our preceding paper, Ref. [13], for simplicity we introduced formal definitions for the matrix elements of the scalar quark and gluon operators which are sufficient for the derivation of evolution equations. There we introduced the following two representations referring to different kinematic variables,

$$
\begin{aligned}
\left\langle p_{2}\left|O^{q, \operatorname{twist} 2}\left(\kappa_{1}, \kappa_{2}\right)\right| p_{1}\right\rangle & =e^{i \kappa_{+} \tilde{x} p_{-}}\left(i \tilde{x} p_{+}\right) \int D z e^{-i \kappa_{-}\left(\tilde{x} p_{i}\right) z_{i}} \hat{f}_{(1)}^{q}\left(z_{+}, z_{-}\right), \\
\left\langle p_{2}\left|O^{G, \operatorname{twist} 2}\left(\kappa_{1}, \kappa_{2}\right)\right| p_{1}\right\rangle & =e^{i \kappa_{+} \tilde{x} p_{-}}\left(i \tilde{x} p_{+}\right)^{2} \int D z e^{-i \kappa_{-}\left(\tilde{x} p_{i}\right) z_{i}} \hat{f}_{(1)}^{G}\left(z_{+}, z_{-}\right),
\end{aligned}
$$

which are now to be replaced by the explicitly determined expressions Eqs. (4.4) and (4.16) projected onto the light-cone. In the limit of forward scattering these functions describe the hadronic parton densities.

However, contrary to the case of the quark operators, for the matrix element of the gluon operator there exists no natural kinematic decomposition. A second parametrization used earlier is

$$
\begin{aligned}
\kappa_{-}\left\langle p_{2}\left|O^{q, \text { twist2}}\left(\kappa_{1}, \kappa_{2}\right)\right| p_{1}\right\rangle & =e^{i \kappa_{+} \tilde{x} p_{-}} \int D z e^{-i \kappa_{-}\left(\tilde{x} p_{i}\right) z_{i}} \hat{f}_{(2)}^{q}\left(z_{+}, z_{-}\right), \\
\kappa_{-}^{2}\left\langle p_{2}\left|O^{G, \text { twist2}}\left(\kappa_{1}, \kappa_{2}\right)\right| p_{1}\right\rangle & =e^{i \kappa_{+} \tilde{x} p_{-}} \int D z e^{-i \kappa_{-}\left(\tilde{x} p_{i}\right) z_{i}} \hat{f}_{(2)}^{G}\left(z_{+}, z_{-}\right)
\end{aligned}
$$

corresponding to

$$
\begin{aligned}
\kappa_{-}\left\langle p_{2}\left|O^{q, \operatorname{twist} 2}\left(-\kappa_{-} \tilde{x}, \kappa_{-} \tilde{x}\right)\right| p_{1}\right\rangle & =\left(\tilde{x} p_{+}\right)^{-1} \tilde{f}_{J}^{q(2)}\left(\kappa_{-} \tilde{x} p_{+}, \kappa_{-} \tilde{x} p_{-}, p_{1} p_{2}, \mu^{2}\right) \mathcal{Q}^{J}\left(\tilde{x} ; p_{2}, p_{1}\right), \\
\kappa_{-}^{2}\left\langle p_{2}\left|O^{G, \operatorname{twist} 2}\left(-\kappa_{-} \tilde{x}, \kappa_{-} \tilde{x}\right)\right| p_{1}\right\rangle & =\left(\tilde{x} p_{+}\right)^{-1} \tilde{f}_{J}^{G(2)}\left(\kappa_{-} \tilde{x} p_{+}, \kappa_{-} \tilde{x} p_{-}, p_{1} p_{2}, \mu^{2}\right) \mathcal{Q}^{J}\left(\tilde{x} ; p_{2}, p_{1}\right) .
\end{aligned}
$$


Because the vector operators are not used in the following we do not write down the respective representations for their matrix elements.

Whereas the first choice in the case of forward scattering leads to the correct matrix elements being proportional to $(2 \tilde{x} p)^{2}$, the second choice leads to a much simpler form of the anomalous dimensions. Note, that in both cases, up to a trivial reduction of powers of $\kappa_{-}$on both sides, everywhere the combination $\kappa_{-} \tilde{x}$ appears as a natural variable.

Let us point to the fact that for both the twist-2 quark and gluon operators the (pseudo)vector distribution amplitude $F_{J}, F_{J, 5}$ are directly related through Eqs. (4.11) and (4.12) to the (pseudo)scalar distribution amplitude $f_{J}, f_{J, 5}$. Therefore we need not to investigate the renormalization properties of the (axial)vector operators independently: It is sufficient to work out the renormalization of the (pseudo)scalar operators and to derive the renormalization group equations for them only.

\section{Compton scattering amplitude in leading order}

With the prerequisites provided in the preceding Sections we now derive the asymptotic representation of the Compton scattering amplitude. Again, we consider the contributions of twist -2 operators only and we restrict the analysis to leading order. Starting from the matrix elements of the non-local operators which depend on the two independent variables $z_{ \pm}$we arrive, after performing the Fourier transformation of Eq. (1.2), at double-variable distributions. ${ }^{4}$ In the literature also single-variable non-forward evolution equations are studied by imposing an additional kinematic constraint. The latter description is suited for special kinematic situations.

\subsection{Double-variable distributions}

\subsubsection{Non-forward scattering}

To obtain the double-variable distributions we use the decomposition Eq. (4.10) of the Fourier transform of the Compton amplitude

$$
T_{\mu \nu}\left(p_{+}, p_{-}, q\right)=i \int d^{4} x e^{i q x}\left\langle p_{2}, S_{2}\left|R T\left(J_{\mu}(x / 2) J_{\nu}(-x / 2) S\right)\right| p_{1}, S_{1}\right\rangle .
$$

Afterwards we have to carry out the limit $x \rightarrow \tilde{x}$. In the r.h.s. of Eq. (5.1) the representation of Eqs. (2.12 2.17) is used. Furthermore the matrix elements are expressed in terms of hadron states, Eqs. (4.4,4.6). The arguments $\kappa_{ \pm}$of the coefficient functions $C_{a(5)}\left(x^{2}, \kappa_{+}, \kappa_{-}, \mu^{2}\right)$ in Eqs. (2.14, 2.15) are fixed at $\kappa_{+}=0$ and $\kappa_{-}= \pm 1 / 2$. For the representation of the Compton amplitude we could use the matrix elements at these points. However, for the investigation of the evolution of the matrix elements their representation at general values of $\kappa_{+}$and $\kappa_{-}$is needed.

The Fourier transforms of the individual terms are obtained by, cf. [25],

$$
\begin{aligned}
\int \frac{d^{4} x}{2 \pi^{2}} e^{i q x} \frac{x^{\nu}}{\left(x^{2}-i \varepsilon\right)^{2}} & =\frac{q^{\nu}}{q^{2}+i \varepsilon}, \\
\int \frac{d^{4} x}{2 \pi^{2}} e^{i q x} \frac{x^{\nu} x^{\mu}}{\left(x^{2}-i \varepsilon\right)^{2}} & =-i \frac{g^{\nu \mu}}{q^{2}+i \varepsilon}+2 i \frac{q^{\nu} q^{\mu}}{\left(q^{2}+i \varepsilon\right)^{2}},
\end{aligned}
$$

\footnotetext{
${ }^{4}$ The general Lorentz-structure of the Compton amplitude was investigated in Refs. [8, 22], cf. also [23]. Real-photon processes were considered in Ref. 24 before. In general the Compton amplitude consists of 18 basis elements. For $q_{1}^{2}=q_{2}^{2}$ four spin-independent and eight spin-dependent distribution amplitudes contribute [8]. Their number reduces to two and four, respectively, for $q_{1}^{2}=q_{2}^{2}=0$.
} 


$$
\int \frac{d^{4} x}{2 \pi^{2}} e^{i q x} \frac{x^{\nu} x^{\mu} x^{\tau}}{\left(x^{2}-i \varepsilon\right)^{2}}=2 \frac{g^{\nu \mu} q^{\tau}+g^{\mu \tau} q^{\nu}+g^{\tau \nu} q^{\mu}}{\left(q^{2}+i \varepsilon\right)^{2}}-8 \frac{q^{\nu} q^{\mu} q^{\tau}}{\left(q^{2}+i \varepsilon\right)^{3}}
$$

In the generalized Bjorken region one obtains, noting that $\xi=Q^{2} / q p_{+}, \eta=q p_{-} / q p_{+}$,

$$
\begin{aligned}
T_{\mu \nu}^{\mathrm{twist} 2}\left(p_{+}, p_{-}, q\right)=\int & D z\left\{\left(\frac{1}{\xi+z_{+}+\eta z_{-}-i \varepsilon}-\frac{1}{\xi-z_{+}-\eta z_{-}-i \varepsilon}\right) F_{\mu \nu}^{(1)}\right. \\
& +\left(\frac{1}{\left(\xi+z_{+}+\eta z_{-}-i \varepsilon\right)^{2}}+\frac{1}{\left(\xi-z_{+}-\eta z_{-}-i \varepsilon\right)^{2}}\right) F_{\mu \nu}^{(2)} \\
& +\left(\frac{1}{\left(\xi+z_{+}+\eta z_{-}-i \varepsilon\right)^{3}}-\frac{1}{\left(\xi-z_{+}-\eta z_{-}-i \varepsilon\right)^{3}}\right) F_{\mu \nu}^{(3)} \\
& +\left(\frac{1}{\xi+z_{+}+\eta z_{-}-i \varepsilon}+\frac{1}{\xi-z_{+}-\eta z_{-}-i \varepsilon}\right) F_{5, \mu \nu}^{(1)} \\
& \left.+\left(\frac{1}{\left(\xi+z_{+}+\eta z_{-}-i \varepsilon\right)^{2}}-\frac{1}{\left(\xi-z_{+}-\eta z_{-}-i \varepsilon\right)^{2}}\right) F_{5, \mu \nu}^{(2)}\right\} .
\end{aligned}
$$

In this representation of $T_{\mu \nu}$ we refer to the essential terms only. One may generalize Eq. (5.2) accounting for current conservation explicitly. For the case of forward scattering this representation was given in Ref. [26].

The functions $F_{(5) \mu \nu}^{(k)}\left(p_{+}, p_{-}, q ; z_{+}, z_{-}\right)$being (anti)symmetric w.r.t. the Lorentz indices are given by

$$
\begin{aligned}
F_{\mu \nu}^{(1)} & =\frac{1}{q p_{+}}\left[q^{\alpha} g_{\mu \nu}-\left(g_{\nu}^{\alpha} q_{\mu}+g_{\mu}^{\alpha} q_{\nu}\right)\right] \mathcal{Q}_{\alpha}^{J}\left(p_{2}, p_{1}\right) F_{J}\left(z_{+}, z_{-}\right) \\
F_{\mu \nu}^{(2)} & =-\left(\frac{1}{q p_{+}}\right)^{2}\left[g_{\mu \nu} q p(z)-\left(q_{\mu} p_{\nu}(z)+q_{\nu} p_{\mu}(z)\right)\right] q^{\alpha} \mathcal{Q}_{\alpha}^{J}\left(p_{2}, p_{1}\right) F_{J}\left(z_{+}, z_{-}\right), \\
F_{\mu \nu}^{(3)} & =8 q_{\mu} q_{\nu}\left(\frac{1}{q p_{+}}\right)^{3} q^{\alpha} \mathcal{Q}_{\alpha}^{J}\left(p_{2}, p_{1}\right) F_{J}^{\prime}\left(z_{+}, z_{-}\right) \\
F_{5, \mu \nu}^{(1)} & =i \varepsilon_{\mu \nu}{ }^{\alpha \beta} q_{\alpha} \frac{1}{q p_{+}} \mathcal{Q}_{\beta}^{J, 5} F_{J, 5}\left(z_{+}, z_{-}\right) \\
F_{5, \mu \nu}^{(2)} & =-i \varepsilon_{\mu \nu}{ }^{\gamma \beta} q_{\gamma} p_{\beta}(z)\left(\frac{1}{q p_{+}}\right)^{2} q^{\alpha} \mathcal{Q}_{\alpha}^{J, 5}\left(p_{2}, p_{1}\right) F_{J, 5}\left(z_{+}, z_{-}\right)
\end{aligned}
$$

where $p(z)$ is defined in Eq. (4.8). Let us remind that the functions $F_{J,(5)}$ are taken in the limit $x^{2} \rightarrow 0$ and that $F_{J}^{\prime}\left(z_{+}, z_{-}\right)$results from the Fourier transformation of $\partial_{\kappa_{-}^{2} x^{2}} F$. The distribution amplitudes $F_{J,(5)}$ and $F_{J}^{\prime}$ are complex quantities in general. The representation of the LCE, Eq. (2.3), implies due to its construction that the current conservation for the incoming and outgoing photon

$$
q_{2 \mu} T^{\mu \nu}=0, \quad T^{\mu \nu} q_{1 \nu}=0
$$

is not obeyed in explicit form yet. However, one may impose these conditions.

\subsubsection{The forward scattering limit}

In the limit of forward scattering, $p_{-}=p_{2}-p_{1} \rightarrow 0, \eta=0$, the Compton amplitude does not depend on the distribution amplitudes $F_{P,(5)}\left(z_{+}, z_{-}\right)$anymore. Using the normalizations

$$
\bar{u}(p) u(p)=2 M, \quad \bar{u}(p) \gamma_{\mu} u(p)=2 p_{\mu}
$$


for the free hadronic Dirac spinors we obtain for the symmetric part of the Compton amplitude

$$
\begin{aligned}
T_{\mu \nu}^{\mathrm{sym}}= & \left(g^{\mu \nu}-\frac{p^{\mu} q^{\nu}+p^{\nu} q^{\mu}}{p q}\right) \int D z \\
& \times\left\{\left(\frac{1}{\xi+z_{+}-i \varepsilon}-\frac{1}{\xi-z_{+}-i \varepsilon}\right)-z_{+}\left(\frac{1}{\left(\xi+z_{+}-i \varepsilon\right)^{2}}+\frac{1}{\left(\xi-z_{+}-i \varepsilon\right)^{2}}\right)\right\} G^{q}\left(z_{+}, z_{-}\right) \\
+ & 2 q_{\mu} q_{\nu} \int D z\left(\frac{1}{\left(\xi+z_{+}-i \varepsilon\right)^{3}}-\frac{1}{\left(\xi-z_{+}-i \varepsilon\right)^{3}}\right) G^{\prime q}\left(z_{+}, z_{-}\right) .
\end{aligned}
$$

The $z_{-}$-integral can be performed

$$
\begin{aligned}
\int_{-1+\left|z_{+}\right|}^{+1-\left|z_{+}\right|} d z_{-} G^{q}\left(z_{+}, z_{-}\right) & =\int_{-1+\left|z_{+}\right|}^{+1-\left|z_{+}\right|} d z_{-} \int_{0}^{1} \frac{d \lambda}{\lambda^{2}} g^{q}\left(\frac{z_{+}}{\lambda}, \frac{z_{-}}{\lambda}\right) \Theta\left(\lambda-\left|z_{+}\right|\right) \Theta\left(\lambda-\left|z_{-}\right|\right) \\
& =\int_{0}^{1} \frac{d \lambda}{\lambda} \int d\left(\frac{z_{-}}{\lambda}\right) g^{q}\left(\frac{z_{+}}{\lambda}, \frac{z_{-}}{\lambda}\right) \Theta\left(\lambda-\left|z_{+}\right|\right) \\
& \equiv \int_{z_{+}}^{\operatorname{sign}\left(z_{+}\right)} \frac{d z^{\prime}}{z^{\prime}} \widehat{g}^{q}\left(z^{\prime}\right) .
\end{aligned}
$$

After partial integration of Eq. (5.8),

$$
\int_{-1}^{+1} d z_{+} \frac{z_{+}}{\left(\xi \pm z_{+}-i \varepsilon\right)^{2}} \int_{z_{+}}^{\operatorname{sign}\left(z_{+}\right)} \frac{d z}{z} \widehat{g}^{q}(z)= \pm \int_{-1}^{+1} d z_{+} \frac{1}{\xi \pm z_{+}-i \varepsilon}\left[\int_{z_{+}}^{\operatorname{sign}\left(z_{+}\right)} \frac{d z}{z} \widehat{g}^{q}(z)-\widehat{g}^{q}\left(z_{+}\right)\right]
$$

we finally obtain

$$
\begin{aligned}
T_{\mu \nu}^{\mathrm{sym}} & =\left(g^{\mu \nu}-\frac{p^{\mu} q^{\nu}+p^{\nu} q^{\mu}}{p q}\right) \int_{-1}^{1} d z_{+}\left(\frac{1}{\xi+z_{+}-i \varepsilon}-\frac{1}{\xi-z_{+}-i \varepsilon}\right) \widehat{g}^{q}\left(z_{+}\right) \\
& +2 q_{\mu} q_{\nu} \int D z\left(\frac{1}{\left(\xi+z_{+}-i \varepsilon\right)^{3}}-\frac{1}{\left(\xi-z_{+}-i \varepsilon\right)^{3}}\right) G^{\prime q}\left(z_{+}, z_{-}\right) .
\end{aligned}
$$

In the same way we can treat the antisymmetric part of the Compton amplitude. Using the normalization condition

$$
\bar{u}(p) \gamma_{5} \gamma_{\beta} u(p)=-2 S_{\beta}, \quad S^{2}=-M^{2}
$$

we obtain analogously

$$
\begin{aligned}
T_{\mu \nu}^{\text {antisym }}= & i \varepsilon_{\mu \nu}^{\gamma \beta} \frac{q_{\gamma} p_{\beta}}{(p q)^{2}} q S \int_{-1}^{+1} d z_{+}\left[\frac{1}{\xi+z_{+}-i \varepsilon}+\frac{1}{\xi-z_{+}-i \varepsilon}\right]\left[\int_{z_{+}}^{\operatorname{sign}\left(z_{+}\right)} \frac{d z}{z} \widehat{g}_{5}^{q}(z)-\widehat{g}_{5}^{q}\left(z_{+}\right)\right] \\
& -i \varepsilon_{\mu \nu} \gamma \beta \frac{q_{\gamma} S_{\beta}}{(p q)} \int_{-1}^{+1} d z_{+}\left[\frac{1}{\xi+z_{+}-i \varepsilon}+\frac{1}{\xi-z_{+}-i \varepsilon}\right] \int_{z_{+}}^{\operatorname{sign}\left(z_{+}\right)} \frac{d z}{z} \widehat{g}_{5}^{q}(z)
\end{aligned}
$$

\subsubsection{Relations between structure functions}

The hadronic tensor is related to the Compton amplitude for forward scattering by

$$
W_{\mu \nu}=\frac{1}{2 \pi} \operatorname{Im} T_{\mu \nu}
$$


In the case of purely electromagnetic interactions the Lorentz-structure of the hadronic tensor is

$$
\begin{aligned}
W_{\mu \nu}= & -g_{\mu \nu} F_{1}\left(x, Q^{2}\right)+\frac{p_{\mu} p_{\nu}}{p q} F_{2}\left(x, Q^{2}\right)+\frac{q_{\mu} q_{\nu}}{p q} F_{4}\left(x, Q^{2}\right)+\frac{\left(p_{\mu} q_{\nu}+q_{\nu} p_{\mu}\right)}{p q} F_{5}\left(x, Q^{2}\right) \\
& +i \varepsilon_{\mu \nu \lambda \sigma} \frac{q^{\lambda} S^{\sigma}}{p q} g_{1}\left(x, Q^{2}\right)+i \varepsilon_{\mu \nu \lambda \sigma} \frac{q^{\lambda}\left(p q S^{\sigma}-S q p^{\sigma}\right)}{(p q)^{2}} g_{2}\left(x, Q^{2}\right)
\end{aligned}
$$

where $x \equiv x_{\mathrm{Bj}}=\xi$. Whereas the polarized structure functions in Eq. (5.13) form already the minimal set, current conservation

$$
q^{\mu} W_{\mu \nu}=W_{\mu \nu} q^{\nu}=0
$$

relates the structure functions $F_{4}$ and $F_{5}$ to $F_{1}$ and $F_{2}$ by

$$
\begin{aligned}
& F_{4}\left(x, Q^{2}\right)=\frac{1}{4 x^{2}}\left[2 x F_{1}\left(x, Q^{2}\right)+F_{2}\left(x, Q^{2}\right)\right], \\
& F_{5}\left(x, Q^{2}\right)=\frac{1}{2 x} F_{2}\left(x, Q^{2}\right) .
\end{aligned}
$$

The forward Compton amplitude Eqs. (5.8) and (5.11) are related to the hadronic tensor Eq. (5.13) using 25

$$
\lim _{\varepsilon \rightarrow 0^{+}} \frac{1}{(x \pm i \varepsilon)^{n}}=\mathrm{P} \frac{1}{x^{n}} \pm(-1)^{n} \frac{i \pi}{(n-1) !} \delta^{(n-1)}(x) .
$$

We now assume that $\widehat{g}_{(5)}^{q}\left(z_{+}\right)$are real functions. The tensor structure of Eq. (5.10) implies

$$
F_{1}\left(x, Q^{2}\right)=F_{5}\left(x, Q^{2}\right)
$$

at leading order, from which, cf. Eq. (5.16), the Callan-Gross relation [21]

$$
F_{2}\left(x, Q^{2}\right)=2 x F_{1}\left(x, Q^{2}\right)
$$

follows. Furthermore, one finds

$$
F_{4}\left(x, Q^{2}\right)=\frac{1}{x} F_{1}\left(x, Q^{2}\right),
$$

which relates the distributions $G^{\prime q}\left(z_{+}, z_{-}\right)$and $G^{q}\left(z_{+}, z_{-}\right)$for forward scattering at leading order. The structure function $F_{1}$ obtains the representation

$$
F_{1}\left(x, Q^{2}\right)=\frac{1}{2}\left[\widehat{g}^{q}(x)-\widehat{g}^{q}(-x)\right] .
$$

We suppressed, as in the preceding Section, the scale dependence of the functions $\widehat{g}_{(5)}^{q}$. In lowest order of perturbation theory the $Q^{2}$-dependence of the structure functions results from the identification $\mu^{2}=Q^{2}$. One may identify this representation with results being obtained in the quark-parton model at leading order by defining

$$
\begin{aligned}
\widehat{g}^{q}(x) & =\sum_{q=1}^{N_{f}} e_{q}^{2} q(x) \\
-\widehat{g}^{q}(-x) & =\sum_{q=1}^{N_{f}} e_{q}^{2} \bar{q}(x) .
\end{aligned}
$$


Here $q(x)$ and $\bar{q}(x)$ denote the unpolarized quark and antiquark densities, respectively, and $e_{q}$ is the electric charge of the quark.

We now turn to the polarized case. The Lorentz structure of the two tensors in Eq. (5.11) refers to the structure functions $g_{1}+g_{2}$ and $g_{2}$, respectively. After performing the $z_{+}$-integration one obtains for the twist-2 terms the Wandzura-Wilczek relation 19

$$
g_{2}\left(x, Q^{2}\right)=-g_{1}\left(x, Q^{2}\right)+\int_{x}^{1} \frac{d z}{z} g_{1}\left(z, Q^{2}\right),
$$

where

$$
g_{1}\left(x, Q^{2}\right)=\frac{1}{2} \sum_{q=1}^{N_{f}} e_{q}^{2}[\Delta q(x)+\Delta \bar{q}(x)]
$$

and we identified

$$
\begin{aligned}
\widehat{g}_{5}^{q}(x) & =\sum_{q=1}^{N_{f}} e_{q}^{2} \Delta q(x) \\
\widehat{g}_{5}^{q}(-x) & =\sum_{q=1}^{N_{f}} e_{q}^{2} \Delta \bar{q}(x) .
\end{aligned}
$$

In the case of general electro-weak couplings also a second integral relation, Ref. [20], is obtained. For both these relations the parameter-integrals relating the vector and scalar operators which are discussed in Section 4 form the background in the present approach. The new derivation of these relations outlined above could circumvent their usual derivation by means of the moments in the local operator product expansion and leads to the integral form directly. We note that recently also three integral relations for the twist-3 contributions to the polarized structure functions $\left.g_{j}\right|_{j=1} ^{5}$ for the case of forward scattering were found in lowest order in the coupling constant, cf. Ref. [26]. Unlike the case for twist-2 [20] all the relations are integral relations requiring an all-order resummation of the $\left(M^{2} / Q^{2}\right)$-terms.

\subsection{Single--variable distributions}

Up to now we have considered representations which contain two distribution parameters $z_{+}, z_{-}$. In some connections another representation may be helpful. As an example let us introduce in representation Eq. (5.2) a new variable $t$ by $z_{+}=t-\eta z_{-}$. Then the integrations factorize and we can write

$$
\begin{aligned}
T_{\mu \nu}^{\mathrm{twist} 2} & \left(p_{+}, p_{-}, Q\right)=\int_{-\infty}^{+\infty} d t\left\{\left(\frac{1}{\xi+t-i \varepsilon}-\frac{1}{\xi-t-i \varepsilon}\right) \hat{F}_{\mu \nu}^{(1)}\left(p_{+}, p_{-}, q ; t, \eta\right)\right. \\
& +\left(\frac{1}{(\xi+t-i \varepsilon)^{2}}+\frac{1}{(\xi-t-i \varepsilon)^{2}}\right) \hat{F}_{\mu \nu}^{(2)}+\left(\frac{1}{(\xi+t-i \varepsilon)^{3}}-\frac{1}{(\xi-t-i \varepsilon)^{3}}\right) \hat{F}_{\mu \nu}^{(3)} \\
& \left.+\left(\frac{1}{\xi+t-i \varepsilon}+\frac{1}{\xi-t-i \varepsilon}\right) \hat{F}_{5, \mu \nu}^{(1)}+\left(\frac{1}{(\xi+t-i \varepsilon)^{2}}-\frac{1}{(\xi-t-i \varepsilon)^{2}}\right) \hat{F}_{5, \mu \nu}^{(2)}\right\} .
\end{aligned}
$$

Also within the functions $F_{(5), \mu \nu}^{(k)}\left(p_{+}, p_{-}, q, F_{J}\left(z_{+}, z_{-} ; p_{1} p_{2}, \mu^{2}\right) ; \mu^{2}\right)$ the variables $z_{+}, z_{-}$have to be transformed thereby changing these functions to $F_{J(5)}\left(z_{+}=t_{-}-\eta z_{-}, z_{-}\right)$. Then the remaining $z_{-}$-integration can be performed to give

$$
\begin{aligned}
\hat{F}_{J(5)}(t, \eta)= & \int_{-1}^{+1} d z_{-} \Theta\left(1+t-\eta z_{-}+z_{-}\right) \Theta\left(1+t-\eta z_{-}-z_{-}\right) \\
& \times \Theta\left(1-t+\eta z_{-}+z_{-}\right) \Theta\left(1-t+\eta z_{-}-z_{-}\right) F_{J(5)}\left(z_{+}=t-\eta z_{-}, z_{-}\right) .
\end{aligned}
$$


These new partition functions $\hat{F}_{J}$ and $\hat{F}_{J, 5}$ depend on the variables $t$ and $\eta$ and therefore we have $\hat{F}_{(5), \mu \nu}^{(k)}\left(p_{+}, p_{-}, q ; t, \eta ; \mu^{2}\right)=F_{(5) \mu \nu}^{(k)}\left(p_{+}, p_{-}, q, \hat{F}_{J}\left(t, \eta ; \mu^{2}\right) ; \mu^{2}\right)$. Obviously, in the limit of forward scattering we obtain $\hat{F}_{(5), \mu \nu}^{(k)}\left(2 p, 0, q ; t, 0 ; \mu^{2}\right)$. Note that the partition functions $F, F^{\prime}$ and $F_{5}$ are directly related to the partition functions $f, f^{\prime}$ and $f_{5}$ which are defined by the matrix elements of scalar and pseudo-scalar twist-2 quark operators, Eq. (4.4). This demonstrates again that for the description of the virtual Compton scattering, at least in leading order, the properties of the scalar operators are sufficient.

The renormalization of the twist-2 operators will be considered in the following Section. The evolution equations of their expectation values are dealt with in Section 7 .

\section{Operators of twist-2 and their anomalous dimensions}

In this section we discuss the renormalization properties of the twist-2 light-ray operators. As has been shown above we can restrict these studies to the case of scalar operators for which any free tensorial index of the various operators is saturated by multiplying with as many as necessary vectors of $\tilde{x}^{\mu}$. Moreover, there holds the much wider statement that the operators $O_{\sigma}^{a \text {,twist2 }}$ and $O^{a, \text { twist2 }}$ are completely equivalent concerning their renormalization properties. This can be seen in the following way. We consider the renormalization group equation of the vector operator,

$$
\mu^{2} \frac{d}{d \mu^{2}} O_{\sigma}^{a, \text { twist } 2}\left(\kappa_{1} \tilde{x}, \kappa_{2} \tilde{x} ; \mu^{2}\right)=\int_{\kappa_{2}}^{\kappa_{1}} d \kappa_{1}^{\prime} d \kappa_{2}^{\prime} \gamma\left(\kappa_{1}, \kappa_{2} ; \kappa_{1}^{\prime}, \kappa_{2}^{\prime} ; \mu^{2}\right) O_{\sigma}^{a, \text { twist } 2}\left(\kappa_{1}^{\prime} \tilde{x}, \kappa_{2}^{\prime} \tilde{x} ; \mu^{2}\right)
$$

cf. Eq. (6.20). Obviously, the scalar operator

$$
O^{a, \text { twist } 2}\left(\kappa_{1}, \kappa_{2}\right)=\tilde{x}^{\sigma} O_{\sigma}^{a, \text { twist2 }}\left(\kappa_{1}, \kappa_{2}\right)
$$

fulfills exactly the same renormalization group equation with the same anomalous dimension kernel $\gamma\left(\kappa_{1}, \kappa_{2} ; \kappa_{1}^{\prime}, \kappa_{2}^{\prime}\right)$, since the multiplication of both sides by $\tilde{x}^{\sigma}$ commutes both with the differentiation on the left and with the integration on the right hand side. The same conclusion can be drawn for the gluon operators.

Because the renormalization properties of the various twist-2 operators are independent of their special Lorentz structure we restrict ourselves to the scalar operators. Contrary to the previous considerations we will account for the flavor content of the operators because in the flavor singlet case twist-2 quark and gluon operators mix under renormalization. To simplify the subsequent considerations, and the necessary computations of Feynman diagrams, we apply the axial gauge where the phase factors are $U\left(\kappa_{1}, \kappa_{2}\right)=1$.

We consider the following scalar flavor non-singlet (NS) and singlet light-ray operators ${ }^{5}$ :

$$
\begin{aligned}
O^{\mathrm{NS}}\left(\kappa_{1}, \kappa_{2}\right) & =\tilde{x}^{\mu} \frac{i}{2}\left[\bar{\psi}\left(\kappa_{1} \tilde{x}\right) \lambda_{f} \gamma_{\mu} \psi\left(\kappa_{2} \tilde{x}\right)-\bar{\psi}\left(\kappa_{2} \tilde{x}\right) \lambda_{f} \gamma_{\mu} \psi\left(\kappa_{1} \tilde{x}\right)\right] \\
O_{5}^{\mathrm{NS}}\left(\kappa_{1}, \kappa_{2}\right) & =\tilde{x}^{\mu} \frac{i}{2}\left[\bar{\psi}\left(\kappa_{1} \tilde{x}\right) \lambda_{f} \gamma_{5} \gamma_{\mu} \psi\left(\kappa_{2} \tilde{x}\right)+\bar{\psi}\left(\kappa_{2} \tilde{x}\right) \lambda_{f} \gamma_{5} \gamma_{\mu} \psi\left(\kappa_{1} \tilde{x}\right)\right] \\
O^{q}\left(\kappa_{1}, \kappa_{2}\right) & =\tilde{x}^{\mu} \frac{i}{2}\left[\bar{\psi}\left(\kappa_{1} \tilde{x}\right) \gamma_{\mu} \psi\left(\kappa_{2} \tilde{x}\right)-\bar{\psi}\left(\kappa_{2} \tilde{x}\right) \gamma_{\mu} \psi\left(\kappa_{1} \tilde{x}\right)\right] \\
O^{G}\left(\kappa_{1}, \kappa_{2}\right) & =\tilde{x}^{\mu} \tilde{x}^{\nu} \frac{1}{2}\left[F_{\mu}^{a \rho}\left(\kappa_{1} \tilde{x}\right) F_{\nu \rho}^{a}\left(\kappa_{2} \tilde{x}\right)+F_{\mu}^{a \rho}\left(\kappa_{2} \tilde{x}\right) F_{\nu \rho}^{a}\left(\kappa_{1} \tilde{x}\right)\right]
\end{aligned}
$$

\footnotetext{
${ }^{5}$ Possible trace terms vanish and the general dependence $R T[O] S$ on the renormalization procedure is understood but has been omitted here.
} 


$$
\begin{aligned}
O_{5}^{q}\left(\kappa_{1}, \kappa_{2}\right) & =\tilde{x}^{\mu} \frac{i}{2}\left[\bar{\psi}\left(\kappa_{1} \tilde{x}\right) \gamma_{5} \gamma_{\mu} \psi\left(\kappa_{2} \tilde{x}\right)+\bar{\psi}\left(\kappa_{2} \tilde{x}\right) \gamma_{5} \gamma_{\mu} \psi\left(\kappa_{1} \tilde{x}\right)\right] \\
O_{5}^{G}\left(\kappa_{1}, \kappa_{2}\right) & =\tilde{x}^{\mu} \tilde{x}^{\nu} \frac{1}{2}\left[F_{\mu}^{a \rho}\left(\kappa_{1} \tilde{x}\right) \tilde{F}_{\nu \rho}^{a}\left(\kappa_{2} \tilde{x}\right)-F_{\mu}^{a \rho}\left(\kappa_{2} \tilde{x}\right) \tilde{F}_{\nu \rho}^{a}\left(\kappa_{1} \tilde{x}\right)\right] .
\end{aligned}
$$

Here $\lambda_{f}$ denotes the generators of the flavor group $S U\left(N_{f}\right)$, where $N_{f}$ is the number of active quark flavors. The scalar operators Eqs. 6.3, 6.5) and (6.6) contribute in the case of unpolarized Compton scattering, whereas the pseudo-scalar operators Eqs. (6.4, 6.7) and (6.8) are relevant for Compton scattering off polarized hadrons.

\subsection{General properties of non-local anomalous dimensions}

We consider now some general properties of the anomalous dimensions of these operators which hold at any order of perturbation theory and are relevant in the following. In order to cover the general case we refer to the scalar singlet operators denoted by $O^{A}$ with $A=(q, G)$. f

The renormalization group equation under consideration reads:

$$
\mu^{2} \frac{d}{d \mu^{2}} O^{A}\left(\kappa_{1} \tilde{x}, \kappa_{2} \tilde{x} ; \mu^{2}\right)=\int_{\kappa_{2}}^{\kappa_{1}} d \kappa_{1}^{\prime} d \kappa_{2}^{\prime} \gamma^{A B}\left(\kappa_{1}, \kappa_{2}, \kappa_{1}^{\prime}, \kappa_{2}^{\prime} ; \mu^{2}\right) O^{B}\left(\kappa_{1}^{\prime} \tilde{x}, \kappa_{2}^{\prime} \tilde{x} ; \mu^{2}\right) .
$$

$\gamma^{A B}$ denotes the non-local anomalous dimension matrix which, through the strong coupling constant $\alpha_{s}\left(\mu^{2}\right)=g_{s}^{2}\left(\mu^{2}\right) /(4 \pi)$, depends on the renormalization scale $\mu$.ป Analogous relations hold for the pseudo-scalar case. The non-singlet cases are covered by the respective projection in flavor-space of the quark-quark submatrix.

Since the variables $\kappa_{i}$ simply parametrize points on the light-ray these anomalous dimensions are invariant under reparametrizations [28], i.e. under translations and scale transformations. [ $^{8}$

$$
\begin{aligned}
\gamma^{A B}\left(\kappa_{1}, \kappa_{2} ; \kappa_{1}^{\prime}, \kappa_{2}^{\prime}\right) & =\gamma^{A B}\left(\kappa_{1}-\kappa, \kappa_{2}-\kappa ; \kappa_{1}^{\prime}-\kappa, \kappa_{2}^{\prime}-\kappa\right) \\
& =\lambda^{d_{A B}} \gamma^{A B}\left(\lambda \kappa_{1}, \lambda \kappa_{2} ; \lambda \kappa_{1}^{\prime}, \lambda \kappa_{2}^{\prime}\right)
\end{aligned}
$$

where

$$
d_{A B}=2+d_{A}-d_{B}
$$

with $d_{A}$ being the difference of the canonical and the $\kappa$-scale dimension of the operators $O^{A}$. The latter is given by the number of factors $\tilde{x}$ emerging in the operator $O_{A}$, i.e.,

$$
d_{q}=1 \quad \text { and } \quad d_{G}=2
$$

Therefore, the number of relevant $\kappa$-parameters may be reduced by two. We consider two (related) choices in order to normalize the anomalous dimension matrix, shifting by either $\kappa_{1}$ or $\kappa_{+}$and scaling by either $\left(\kappa_{2}-\kappa_{1}\right)^{-1}$ or $\left(\kappa_{-}\right)^{-1}$, respectively. One obtains

$$
\begin{aligned}
\left(\kappa_{2}-\kappa_{1}\right)^{d_{A B}} \gamma^{A B}\left(\kappa_{1}, \kappa_{2} ; \kappa_{1}^{\prime}, \kappa_{2}^{\prime}\right) & =\gamma^{A B}\left(0,1 ; \alpha_{1}, 1-\alpha_{2}\right) \equiv \widehat{K}^{A B}\left(\alpha_{1}, \alpha_{2}\right) \\
4\left(\kappa_{-}\right)^{d_{A B}} \gamma^{A B}\left(\kappa_{1}, \kappa_{2} ; \kappa_{1}^{\prime}, \kappa_{2}^{\prime}\right) & =4 \gamma^{A B}\left(-1,+1 ; w_{1}, w_{2}\right) \equiv \widetilde{K}^{A B}\left(w_{1}-w_{2}, w_{1}+w_{2}\right)
\end{aligned}
$$

\footnotetext{
${ }^{6}$ The respective relations hold synonymously for the three possible cases of non-singlet evolution equations [27] which have to be suitably projected out. They are all equivalent in leading order, and two of them are even equivalent in next-to-leading order.

${ }^{7}$ In the remaining part of this and the following section the explicit dependence on $\tilde{x}$ and $\mu^{2}$ will be suppressed in the operators, anomalous dimensions and evolution kernels.

${ }^{8}$ These properties of the anomalous dimensions may be traced back to the independence of the renormalization properties of the operators from shifting or scaling their arguments.
} 
where

$$
\begin{aligned}
\alpha_{1}=\frac{\kappa_{1}^{\prime}-\kappa_{1}}{\kappa_{2}-\kappa_{1}}, & -\alpha_{2}=\frac{\kappa_{2}^{\prime}-\kappa_{2}}{\kappa_{2}-\kappa_{1}}, \\
w_{1}=\alpha_{1}-\alpha_{2}=\frac{\kappa_{+}^{\prime}-\kappa_{+}}{\kappa_{-}}, & w_{2}=1-\alpha_{1}-\alpha_{2}=\frac{\kappa_{-}^{\prime}}{\kappa_{-}},
\end{aligned}
$$

respectively. The variables $\kappa^{\prime}$ are related to the parameters $\alpha$ and $w$ by

$$
\left(\begin{array}{l}
\kappa_{1}^{\prime} \\
\kappa_{2}^{\prime}
\end{array}\right)=\left(\begin{array}{l}
\kappa_{1}\left(1-\alpha_{1}\right)+\kappa_{2} \alpha_{1} \\
\kappa_{2}\left(1-\alpha_{2}\right)+\kappa_{1} \alpha_{2}
\end{array}\right)=\frac{1}{2}\left(\begin{array}{l}
\kappa_{1}\left(1-w_{1}+w_{2}\right)+\kappa_{2}\left(1+w_{1}-w_{2}\right) \\
\kappa_{1}\left(1-w_{1}-w_{2}\right)+\kappa_{2}\left(1+w_{1}+w_{2}\right)
\end{array}\right) .
$$
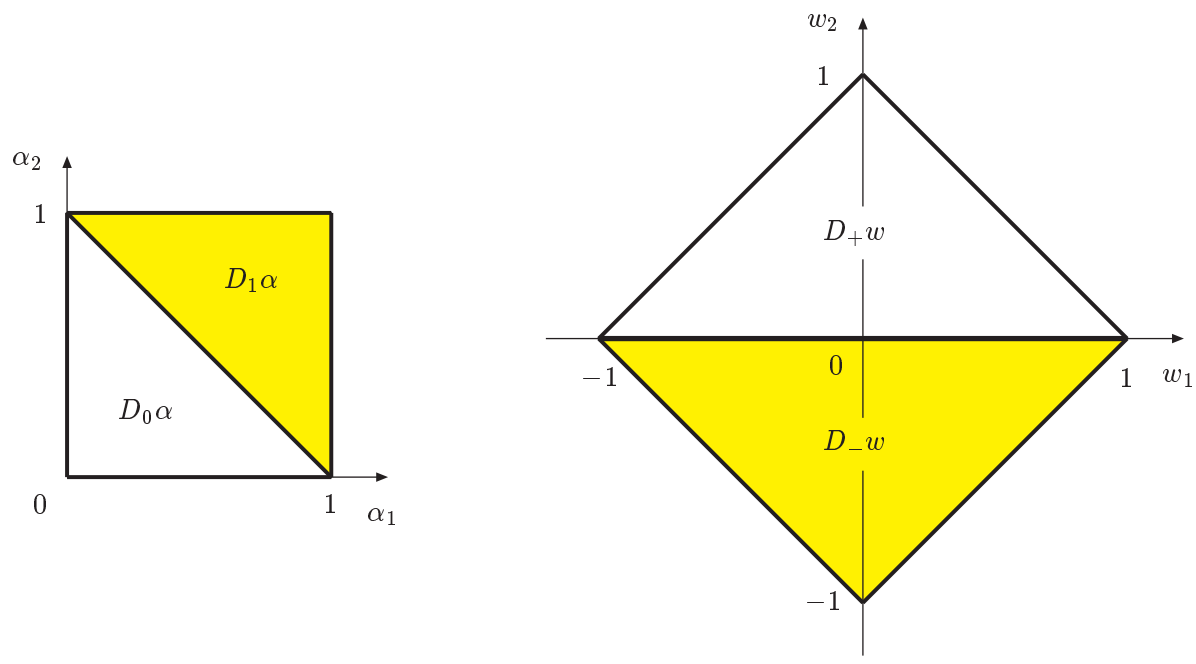

Figure 1: Integration ranges of the parameters $\left(\alpha_{1}, \alpha_{2}\right)$ and $\left(w_{1}, w_{2}\right)$, Eqs. (6.21, 6.22).

Therefore, it seems to be quite natural to introduce instead of the variables $\left(\kappa_{1}^{\prime}, \kappa_{2}^{\prime}\right)$ as new variables either $\left(\alpha_{1}, \alpha_{2}\right)$ or $\left(w_{1}, w_{2}\right)$. The integration measures are related by

$$
D \kappa^{\prime}=\left(\kappa_{2}-\kappa_{1}\right)^{2} D \alpha=4\left(\kappa_{-}\right)^{2} D w
$$

where

$$
\begin{aligned}
D \kappa^{\prime} \equiv d \kappa_{1}^{\prime} d \kappa_{2}^{\prime} \Theta\left(\kappa_{1}-\kappa_{1}^{\prime}\right) \Theta\left(\kappa_{1}^{\prime}-\kappa_{2}\right) \Theta\left(\kappa_{1}-\kappa_{2}^{\prime}\right) \Theta\left(\kappa_{2}^{\prime}-\kappa_{2}\right), \\
D \alpha=D_{0} \alpha+D_{1} \alpha \equiv d \alpha_{1} d \alpha_{2}\left[\Theta\left(\alpha_{1}\right) \Theta\left(\alpha_{2}\right) \Theta\left(1-\alpha_{1}-\alpha_{2}\right)\right. \\
\left.+\quad \Theta\left(1-\alpha_{1}\right) \Theta\left(1-\alpha_{2}\right) \Theta\left(\alpha_{1}+\alpha_{2}-1\right)\right], \\
D w=D_{+} w+D_{-} w \equiv \frac{1}{2} d w_{1} d w_{2}\left[\Theta\left(1+w_{1}-w_{2}\right) \Theta\left(1-w_{1}-w_{2}\right) \Theta\left(w_{2}\right)\right. \\
\left.+\quad \Theta\left(1+w_{1}+w_{2}\right) \Theta\left(1-w_{1}+w_{2}\right) \Theta\left(-w_{2}\right)\right] .
\end{aligned}
$$

The $w$-representation was derived in Ref. [5] for all orders in the coupling constant. The measures $D \alpha$ and $D w$ have been divided into two parts related by $\alpha_{i} \leftrightarrow 1-\alpha_{i}$ and $w_{2} \leftrightarrow-w_{2}$, respectively. Usually only the first part (unshadowed in Fig. 1) is considered in the literature. Because of symmetry requirements for the anomalous dimensions being discussed below this more general representation proves, however, to be appropriate. 
Let us now consider the symmetry of the quark and gluon operators, and likewise of their anomalous dimensions, under changing $\left(\kappa_{1}, \kappa_{2}\right) \leftrightarrow\left(\kappa_{2}, \kappa_{1}\right)$ and $\left(\kappa_{1}^{\prime}, \kappa_{2}^{\prime}\right) \leftrightarrow\left(\kappa_{2}^{\prime}, \kappa_{1}^{\prime}\right)$. Looking at Eqs. 6.5-6.8) it is easily seen that

$$
\begin{aligned}
& O^{A}\left(\kappa_{1}, \kappa_{2}\right)=(-1)^{d_{A}} O^{A}\left(\kappa_{2}, \kappa_{1}\right), \\
& O_{5}^{A}\left(\kappa_{1}, \kappa_{2}\right)=-(-1)^{d_{A}} O_{5}^{A}\left(\kappa_{2}, \kappa_{1}\right),
\end{aligned}
$$

holds. We first consider the scalar case. From Eq. (6.23) we obtain

$$
\gamma^{A B}\left(\kappa_{1}, \kappa_{2} ; \kappa_{1}^{\prime}, \kappa_{2}^{\prime}\right)=(-1)^{d_{A B}} \gamma^{A B}\left(\kappa_{2}, \kappa_{1} ; \kappa_{2}^{\prime}, \kappa_{1}^{\prime}\right)=(-1)^{d_{B}} \gamma^{A B}\left(\kappa_{1}, \kappa_{2} ; \kappa_{2}^{\prime}, \kappa_{1}^{\prime}\right) .
$$

Using reparametrization invariance, Eqs. (6.10) and (6.11), we obtain

$$
\begin{aligned}
& \widehat{K}^{A B}\left(\alpha_{1}, \alpha_{2}\right)=\widehat{K}^{A B}\left(\alpha_{2}, \alpha_{1}\right)=(-1)^{d_{B}} \widehat{K}^{A B}\left(1-\alpha_{1}, 1-\alpha_{2}\right) \\
& \widetilde{K}^{A B}\left(w_{1}, w_{2}\right)=\widetilde{K}^{A B}\left(-w_{1}, w_{2}\right)=(-1)^{d_{B}} \widetilde{K}^{A B}\left(w_{1},-w_{2}\right) .
\end{aligned}
$$

The renormalization group equation (6.9) transforms into

$$
\begin{aligned}
\mu^{2} \frac{d}{d \mu^{2}} O^{A}\left(\kappa_{1}, \kappa_{2}\right) & =\int D \alpha\left(\kappa_{2}-\kappa_{1}\right)^{d_{B}-d_{A}} \widehat{K}^{A B}\left(\alpha_{1}, \alpha_{2}\right) O^{B}\left(\kappa_{1}^{\prime}, \kappa_{2}^{\prime}\right) \\
\mu^{2} \frac{d}{d \mu^{2}} O^{A}\left(\kappa_{1}, \kappa_{2}\right) & =\int D w\left(\kappa_{-}\right)^{d_{B}-d_{A}} \widetilde{K}^{A B}\left(w_{1}, w_{2}\right) O^{B}\left(\kappa_{1}^{\prime}, \kappa_{2}^{\prime}\right) \\
& =\int_{0}^{1} d w_{2} \int_{-1+w_{2}}^{1-w_{2}} d w_{1}\left(\kappa_{-}\right)^{d_{B}-d_{A}} \widetilde{K}_{\mathrm{sym}}^{A B}\left(w_{1}, w_{2}\right) O^{B}\left(\kappa_{1}^{\prime}, \kappa_{2}^{\prime}\right)
\end{aligned}
$$

using the kernels $\widehat{K}^{A B}\left(\alpha_{1}, \alpha_{2}\right)$ and $\widetilde{K}^{A B}\left(w_{1}, w_{2}\right)$, Eqs. (6.14, 6.15).9 In the second line of Eq. (6.29) we have taken into account the above decomposition (6.22) of the integration measure and the second of the equalities (6.27) to restrict the integration onto the usual range by symmetrizing the anomalous dimension kernels in an appropriate way:

$$
\widetilde{K}_{\mathrm{sym}}^{A B}\left(w_{1}, w_{2}\right)=\frac{1}{2}\left[\widetilde{K}_{0}^{A B}\left(w_{1}, w_{2}\right)+(-1)^{d_{B}} \widetilde{K}_{0}^{A B}\left(w_{1},-w_{2}\right)\right] .
$$

Here $\widetilde{K}_{0}^{A B}\left(w_{1}, w_{2}\right)$ is defined in the range of the measure $D_{+} w$ only. Even more, with the help of the first of the equalities (6.27) the $w_{1}$-integration can be restricted to the range $0 \leq w_{1} \leq 1-w_{2}$. Using these kernels we can write the renormalization group equation for the centered scalar operators in terms of the variables $w$ as follows:

$$
\mu^{2} \frac{d}{d \mu^{2}} O^{A}\left(-\kappa_{-}, \kappa_{-}\right)=2 \int_{0}^{1} d w_{2} \int_{0}^{1-w_{2}} d w_{1} \kappa_{-}^{d_{B}-d_{A}} \widetilde{K}_{\mathrm{sym}}^{A B}\left(w_{1}, w_{2}\right) O^{B}\left(\left(w_{1}-w_{2}\right) \kappa_{-},\left(w_{1}+w_{2}\right) \kappa_{-}\right) .
$$

In terms of the $\alpha$-variables these expressions are more lengthy.

In the pseudo-scalar case the sign in the last expression of Eqs. (6.25-6.27), as well as in Eq. (6.30) has to be changed into $-(-1)^{d_{B}}$ in accordance with Eq. (6.24), which also implies the behavior of the sign functions $\sigma_{n n^{\prime}}^{( \pm)}$defined in Eq. (6.52) below. The corresponding kernels will be denoted by $\Delta \gamma, \Delta \widehat{K}$ and $\Delta \widehat{K}$, respectively.

\footnotetext{
${ }^{9}$ To get rid of the unwanted $\kappa$-dependent factors in these equations, it seems to be reasonable to multiply the scalar quark and gluon operators $O^{A}$ by $\left(\kappa_{2}-\kappa_{1}\right)^{d_{A}}$ or $\kappa_{-}^{d_{A}}$ if the $\alpha$ - or $w$-representation has been chosen. However, this does not really resolve the problem since an additional factor $\left(1-\alpha_{1}-\alpha_{2}\right)^{-d_{B}}$ or $w_{2}^{-d_{B}}$ is introduced which multiplies the kernels $\widehat{K}$ and $\widetilde{K}$, respectively.
} 


\subsection{Relations between non-local and local anomalous dimensions}

Obviously, the general statements proven here for the non-local operators and their anomalous dimension kernels can be formulated for the local operators and their anomalous dimension matrices, too. With the definition Eq. (2.9) of the local operators $O_{n_{1} n_{2}}^{\Gamma}$ the renormalization group equations read

$$
\mu^{2} \frac{d}{d \mu^{2}} O_{n_{1} n_{2}}^{A}=\sum_{n_{1}^{\prime}, n_{2}^{\prime}} \gamma_{n_{1}, n_{2} ; n_{1}^{\prime}, n_{2}^{\prime}}^{A B} O_{n_{1}^{\prime} n_{2}^{\prime}}^{B}
$$

with

$$
\gamma_{n_{1}, n_{2} ; n_{1}^{\prime}, n_{2}^{\prime}}^{A B}=\left.\frac{\partial^{n_{1}}}{\partial \kappa_{1}^{n_{1}}} \frac{\partial^{n_{2}}}{\partial \kappa_{2}^{n_{2}}} \int_{\kappa_{2}}^{\kappa_{1}} d \kappa_{1}^{\prime} \int_{\kappa_{2}}^{\kappa_{1}} d \kappa_{2}^{\prime} \frac{\left(\kappa_{1}^{\prime}\right)^{n_{1}^{\prime}}}{n_{1}^{\prime} !} \frac{\left(\kappa_{2}^{\prime}\right)^{n_{2}^{\prime}}}{n_{2}^{\prime} !} \gamma^{A B}\left(\kappa_{1}, \kappa_{2} ; \kappa_{1}^{\prime}, \kappa_{2}^{\prime}\right)\right|_{\kappa_{1}=\kappa_{2}=0} .
$$

First of all we remark that the symmetry relation $(6.25)$ is simply transformed into

$$
\gamma_{n_{1}, n_{2} ; n_{1}^{\prime}, n_{2}^{\prime}}^{A B}=(-1)^{d_{A B}} \gamma_{n_{2}, n_{1} ; n_{2}^{\prime}, n_{1}^{\prime}}^{A B}=(-1)^{d_{B}} \gamma_{n_{1}, n_{2} ; n_{2}^{\prime}, n_{1}^{\prime}}^{A B}
$$

Furthermore, taking into account the reparametrization invariance, Eqs. (6.14) or (6.15), together with the corresponding representation of $\kappa_{i}^{\prime}$, it is easily seen that the values of $n_{i}$ and $n_{i}^{\prime}$ are restricted by the relations

$$
N=n_{1}+n_{2}=n_{1}^{\prime}+n_{2}^{\prime}+d_{B}-d_{A}
$$

since otherwise the anomalous dimension vanishes. From this it is obvious that only those local operators mix under renormalization which are specified by $N$. Therefore, we may choose

$$
n_{1}=N-n, \quad n_{2}=n ; \quad n_{1}^{\prime}=N-n^{\prime}, \quad n_{2}^{\prime}=n^{\prime}-d_{B}+d_{A},
$$

and the local anomalous dimension can be written as

$$
\gamma_{n_{1}, n_{2} ; n_{1}^{\prime}, n_{2}^{\prime}}^{A B}=\gamma_{N-n, n ; N-n^{\prime}, n^{\prime}-d_{B}+d_{A}}^{A B} \equiv{ }^{N} \gamma_{n n^{\prime}}^{A B} .
$$

Now we go over to the variables $\kappa_{ \pm}$in place of $\left(\kappa_{1}, \kappa_{2}\right)$ which are favored because of the relations (6.15) and (6.17). For simplicity we perform the next steps for the non-singlet local anomalous dimensions only. Analogous results are obtained in the singlet case. Let us define

$$
\begin{aligned}
\widetilde{O}_{n_{1} n_{2}} & =\left.\partial_{+}^{n_{1}} \partial_{-}^{n_{2}} O\left(\kappa_{1} \tilde{x}, \kappa_{2} \tilde{x}\right)\right|_{\kappa_{ \pm}=0}, \\
\widetilde{\gamma}_{n_{1}, n_{2} ; n_{1}^{\prime}, n_{2}^{\prime}} & =\left.\partial_{+}^{n_{1}} \partial_{-}^{n_{2}} \int D w \frac{\left(\kappa_{+}^{\prime}\right)^{n_{1}^{\prime}}}{n_{1}^{\prime} !} \frac{\left(\kappa_{-}^{\prime}\right)^{n_{2}^{\prime}}}{n_{2}^{\prime} !} \widetilde{K}\left(w_{1}, w_{2}\right)\right|_{\kappa_{ \pm}=0}
\end{aligned}
$$

with the abbreviation $\partial_{ \pm} \equiv \partial / \partial \kappa_{ \pm}$. Taking into account the representation of $\kappa_{ \pm}^{\prime}$ by Eq. (6.17) and decomposing $\left(\kappa_{+}^{\prime}\right)^{n_{1}^{\prime}}=\left(\kappa_{+}+w_{1} \kappa_{-}\right)^{n_{1}^{\prime}}$ we obtain

$$
\begin{aligned}
{ }^{N} \gamma_{n n^{\prime}} \equiv \gamma_{n n^{\prime}} & =\frac{\partial_{+}^{N-n} \partial_{-}^{n}}{\left(N-n^{\prime}\right) ! n^{\prime} !} \sum_{l=0}^{N-n^{\prime}}\left(\begin{array}{c}
N-n^{\prime} \\
l
\end{array}\right) \kappa_{+}^{N-n^{\prime}-l} \kappa_{-}^{l+n^{\prime}} \int D w w_{1}^{l} w_{2}^{n^{\prime}} \widetilde{K}\left(w_{1}, w_{2}\right) \\
& =\left(\begin{array}{c}
n \\
n^{\prime}
\end{array}\right) \int D w w_{1}^{n-n^{\prime}} w_{2}^{n^{\prime}} \widetilde{K}\left(w_{1}, w_{2}\right) .
\end{aligned}
$$

From this equation some remarkable facts can be read off which hold at any order of perturbation theory. First of all, the local matrix of anomalous dimensions is triangular, i.e. $n^{\prime} \leq n$ and, 
secondly, it is universal, i.e., independent of $N$. Thirdly, because in the case of forward scattering, $p_{-}=0$, the variable $\kappa_{+}$disappears from the expressions, cf. Eq. (4.3), the relation $N=n=n^{\prime}$ is implied with the consequence that the diagonal elements of that matrix are the forward anomalous dimensions. In the unpolarized case one obtains

$$
\gamma_{n}=\frac{1}{2}\left(1-(-1)^{n}\right) \int_{0}^{1} d w_{2} w_{2}^{n}\left\{2 \int_{0}^{1-w_{2}} d w_{1} \widetilde{K}_{0}\left(w_{1}, w_{2}\right)\right\}
$$

The anomalous dimensions vanish for even values of $n .0$

Since the local anomalous dimensions in the forward case are moments of the splitting functions, the latter are given by

$$
P(z)=2 \int_{0}^{1-z} d w_{1} \widetilde{K}_{0}\left(w_{1}, z\right)
$$

performing the inverse Mellin transformation. Here $z$ denotes the partonic momentum fraction. Analogous relations hold in the pseudo-scalar case, with the difference that now the even values of $n$ are to be taken. These considerations show the value of the $w$-representation, which naturally introduces a partial diagonalization in the variable $w_{2}$ through the Mellin transformation. It becomes also evident that the $w_{1}$-dependence is related to the off-diagonality of the anomalous dimension matrix.

Let us now consider the local anomalous dimensions for the singlet case. The local scalar light-ray operators are given by

$$
\begin{array}{rlrl}
{ }^{N} \widetilde{O}_{n}^{q} & =\left.\partial_{+}^{N-n} \partial_{-}^{n} O^{q}\left(\kappa_{1} \tilde{x}, \kappa_{2} \tilde{x}\right)\right|_{\kappa_{ \pm}=0} \quad \text { with } & 0 \leq n \leq N, \\
{ }^{N} \widetilde{O}_{n-1}^{G} & =\left.\partial_{+}^{N-n} \partial_{-}^{n-1} O^{G}\left(\kappa_{1} \tilde{x}, \kappa_{2} \tilde{x}\right)\right|_{\kappa_{ \pm}=0} & \text { with } & 1 \leq n \leq N,
\end{array}
$$

where $N+1$ equals the total spin of the local operators mixing under renormalization. The difference between quark and gluon operators results from the fact that their $\kappa$-scale dimensions equals $d_{q}=1, d_{G}=2$. Obviously, ${ }^{N} O_{0}^{q}$ has no counterpart to mix with. This situation can be resolved if

$$
\left(\underline{O}^{q}, \underline{O}^{G}\right) \equiv\left(\partial_{-} O^{q}, O^{G}\right)
$$

is used instead of $\left(O^{q}, O^{G}\right)$. The corresponding renormalization group equations read

$$
\mu^{2} \frac{d}{d \mu^{2}}{ }^{N} \underline{O}_{n-1}^{A}=\sum_{n^{\prime}=1}^{n}{ }^{N} \underline{\gamma}_{n-1, n^{\prime}-1}^{A B} \underline{O}_{n^{\prime}-1}^{B} \quad \text { with } \quad 1 \leq n, n^{\prime} \leq N
$$

where the local anomalous dimension matrix ${ }^{N} \underline{\gamma}_{n-1, n^{\prime}-1}^{A B}$ is given by

$$
{ }^{N} \underline{\gamma}_{n-1, n^{\prime}-1}^{A B}=\left(\begin{array}{c}
n-1 \\
n^{\prime}-1
\end{array}\right) \int D w w_{1}^{n-n^{\prime}} w_{2}^{n^{\prime}-1} \underline{\widetilde{K}}^{A B}\left(w_{1}, w_{2}\right)
$$

in accordance with Eq. (6.40). Of course the same conclusions concerning the triangularity, universality and the eigenvalues of these anomalous dimension matrices can be drawn as in the

\footnotetext{
10 The reader should be reminded that usually in the definition of the forward anomalous dimensions, Eq. (6.41), the value of $n$ is shifted to $n-1$. To choose the Mellin transform with the power $(n-1)$ rather than the power $n$ is motivated by the interpretation of this quantity in terms of an angular momentum variable. However, choosing $n$, as we have done here, leads to more compact representations.
} 
non-singlet case above. However, the connection with the kernels $\widetilde{K}^{A B}\left(w_{1}, w_{2}\right)$ by no means is trivial. However, computing the elements of the usual local anomalous dimension matrix $\gamma_{n n^{\prime}}^{A B}$ individually, taking into account that only operators of the same value of $N$ mix under renormalization, one obtains the (infinite) triangular matrices [29]

$$
\begin{aligned}
\gamma_{n n^{\prime}}^{q q} & =\left(\begin{array}{c}
n \\
n^{\prime}
\end{array}\right) \sigma_{n n^{\prime}}^{(-)} \int_{0}^{1} d w_{2} w_{2}^{n^{\prime}}\left\{2 \int_{0}^{1-w_{2}} d w_{1} w_{1}^{n-n^{\prime}} \widetilde{K}_{0}^{q q}\left(w_{1}, w_{2}\right)\right\} \\
\gamma_{n n^{\prime}}^{q G} & =n\left(\begin{array}{c}
n-1 \\
n^{\prime}-1
\end{array}\right) \sigma_{n n^{\prime}}^{(-)} \int_{0}^{1} d w_{2} w_{2}^{n^{\prime}-1}\left\{2 \int_{0}^{1-w_{2}} d w_{1} w_{1}^{n-n^{\prime}} \widetilde{K}_{0}^{q G}\left(w_{1}, w_{2}\right)\right\}, \\
\gamma_{n n^{\prime}}^{G q} & =\frac{1}{n}\left(\begin{array}{c}
n \\
n^{\prime}
\end{array}\right) \sigma_{n n^{\prime}}^{(-)} \int_{0}^{1} d w_{2} w_{2}^{n^{\prime}}\left\{2 \int_{0}^{1-w_{2}} d w_{1} w_{1}^{n-n^{\prime}} \widetilde{K}_{0}^{G q}\left(w_{1}, w_{2}\right)\right\} \\
\gamma_{n n^{\prime}}^{G G} & =\left(\begin{array}{c}
n-1 \\
n^{\prime}-1
\end{array}\right) \sigma_{n n^{\prime}}^{(-)} \int_{0}^{1} d w_{2} w_{2}^{n^{\prime}-1}\left\{2 \int_{0}^{1-w_{2}} d w_{1} w_{1}^{n-n^{\prime}} \widetilde{K}_{0}^{G G}\left(w_{1}, w_{2}\right)\right\}
\end{aligned}
$$

with

$$
\sigma_{n n^{\prime}}^{( \pm)}=\frac{1}{4}\left(1+(-1)^{n-n^{\prime}}\right)\left(1 \pm(-1)^{n^{\prime}-2 d_{B}}\right)=\frac{1}{4}\left(1 \pm(-1)^{n}\right)\left(1 \pm(-1)^{n^{\prime}}\right)
$$

and $1 \leq n, n^{\prime} \leq \infty ; \sigma_{n n^{\prime}}^{(+)}$emerges in the pseudo-scalar case. $\square$ The difference of the sign due to $(-1)^{d_{B}}$ is compensated by an additional factor $-(-1)^{d_{B}}$ which results from the different powers of $w_{2}$ in Eqs. (6.48-6.51). The eigenvalues of these triangular matrices are given by their diagonal elements $\gamma_{n n}^{A B}$. In a way similar to Eq. (6.42) the splitting functions in the case of forward scattering are obtained for the singlet case [13, 29]

$$
P^{A B}(z)=2 \int_{0}^{1} d w \widetilde{O}^{A B}(w, z) \int_{0}^{1-w} d w_{1} \widetilde{K}_{0}^{A B}\left(w_{1}, w\right)
$$

(no summation) with

$$
\widetilde{O}^{A B}(w, z)=\left(\begin{array}{cc}
\delta(w-z) & \partial_{w} \delta(w-z) \\
\theta(w-z) / z & \delta(w-z) / z
\end{array}\right) .
$$

With respect to the cross terms an additional differentiation or integration has been introduced to remove the factors $n$ or $1 / n$, followed by partial integration or changing the order of integration, respectively. The consideration of the pseudo-scalar case follows the same line of reasoning.

\subsection{Anomalous dimensions of twist-2 operators at $\mathcal{O}\left(\alpha_{s}\right)$}

After this general exposition we turn to the explicit calculation for the above operators 6.3 6.8) in $\mathcal{O}\left(\alpha_{s}\right)$. We will consider first the unsymmetrized singlet anomalous dimensions $\widehat{K}_{0}^{A B}$ and $\Delta \widehat{K}_{0}^{A B}$ for the unpolarized and the polarized case, respectively, in the $\alpha$-representation. Here the parameters $\left(\alpha_{1}, \alpha_{2}\right)$ are nothing but the usual Feynman parameters appearing in the calculation of the corresponding Feynman diagrams. As far as relations are concerned which are valid both for the unpolarized and polarized case we will, for brevity, only give the results for the unpolarized case in the following. The non-singlet anomalous dimensions obey in leading order

$$
\widehat{K}_{0}^{\mathrm{NS}}=\Delta \widehat{K}_{0}^{\mathrm{NS}}=\widehat{K}_{0}^{q q}=\Delta \widehat{K}_{0}^{q q}
$$

\footnotetext{
${ }^{11}$ Note that according to our above convention on $n$ the local anomalous dimensions are defined in the unpolarized case at odd integers $n$ and $n^{\prime}$, whereas they are defined at even integers in the polarized case in difference to other conventions in the literature.
} 
For brevity we use the convention that the common factors resulting from the order of perturbation theory, as $\alpha_{s} /(2 \pi)$, and from the $\Theta$-structure of the different integration measures will be separated off from the anomalous dimension kernels. Then without introducing new symbols for the reduced kernels $\widehat{K}_{0}^{q q}\left(\alpha_{1}, \alpha_{2}\right)$ we obtain

$$
\begin{aligned}
K_{(1)}^{A B}\left(\alpha_{1}, \alpha_{2}, \kappa_{-}\right) & =\frac{\alpha_{s}\left(\mu^{2}\right)}{2 \pi}\left(\kappa_{2}-\kappa_{1}\right)^{d_{B}-d_{A}}\left(\Theta\left(\alpha_{1}\right) \Theta\left(\alpha_{2}\right) \Theta\left(1-\alpha_{1}-\alpha_{2}\right) \widehat{K}_{0}^{A B}\left(\alpha_{1}, \alpha_{2}\right)\right. \\
& \left.+(-1)^{d_{B}} \Theta\left(1-\alpha_{1}\right) \Theta\left(1-\alpha_{2}\right) \Theta\left(\alpha_{1}+\alpha_{2}-1\right) \widehat{K}_{0}^{A B}\left(1-\alpha_{1}, 1-\alpha_{2}\right)\right) .
\end{aligned}
$$

For the unsymmetrized kernels $\widehat{K}_{0}^{A B}\left(\alpha_{1}, \alpha_{2}\right)$ in leading order we get:

1) Unpolarized anomalous dimensions

$$
\begin{aligned}
\widehat{K}_{0}^{q q}\left(\alpha_{1}, \alpha_{2}\right)= & C_{F}\left\{1-\delta\left(\alpha_{1}\right)-\delta\left(\alpha_{2}\right)+\delta\left(\alpha_{1}\right)\left[\frac{1}{\alpha_{2}}\right]_{+}+\delta\left(\alpha_{2}\right)\left[\frac{1}{\alpha_{1}}\right]_{+}+\frac{3}{2} \delta\left(\alpha_{1}\right) \delta\left(\alpha_{2}\right)\right\} \\
\widehat{K}_{0}^{q G}\left(\alpha_{1}, \alpha_{2}\right)= & -2 N_{f} T_{R}\left\{1-\alpha_{1}-\alpha_{2}+4 \alpha_{1} \alpha_{2}\right\} \\
\widehat{K}_{0}^{G q}\left(\alpha_{1}, \alpha_{2}\right)= & -C_{F}\left\{\delta\left(\alpha_{1}\right) \delta\left(\alpha_{2}\right)+2\right\} \\
\widehat{K}_{0}^{G G}\left(\alpha_{1}, \alpha_{2}\right)= & C_{A}\left\{4\left(1-\alpha_{1}-\alpha_{2}\right)+12 \alpha_{1} \alpha_{2}+\delta\left(\alpha_{1}\right)\left(\left[\frac{1}{\alpha_{2}}\right]_{+}-2+\alpha_{2}\right)\right. \\
& \left.+\delta\left(\alpha_{2}\right)\left(\left[\frac{1}{\alpha_{1}}\right]_{+}-2+\alpha_{1}\right)\right\}+\frac{1}{2} \beta_{0} \delta\left(\alpha_{1}\right) \delta\left(\alpha_{2}\right)
\end{aligned}
$$

where $C_{F}=\left(N_{c}^{2}-1\right) / 2 N_{c} \equiv 4 / 3, T_{R}=1 / 2, C_{A}=N_{c} \equiv 3$, and the $\beta$-function in leading order, $\beta_{0}=\left(11 C_{A}-4 T_{R} N_{f}\right) / 3$. $\square$ The [ $]_{+}$-prescription is defined as

$$
\int_{0}^{1} d x[f(x, y)]_{+} \varphi(x)=\int_{0}^{1} d x f(x, y)[\varphi(x)-\varphi(y)]
$$

if the singularity of $f$ is of the type $\sim 1 /(x-y)$.

2) Polarized anomalous dimensions

$$
\begin{aligned}
\Delta \widehat{K}_{0}^{q q}\left(\alpha_{1}, \alpha_{2}\right) & =\widehat{K}_{0}^{q q}\left(\alpha_{1}, \alpha_{2}\right) \\
\Delta \widehat{K}_{0}^{q G}\left(\alpha_{1}, \alpha_{2}\right) & =-2 N_{f} T_{R}\left\{1-\alpha_{1}-\alpha_{2}\right\} \\
\Delta \widehat{K}_{0}^{G q}\left(\alpha_{1}, \alpha_{2}\right) & =-C_{F}\left\{\delta\left(\alpha_{1}\right) \delta\left(\alpha_{2}\right)-2\right\} \\
\Delta \widehat{K}_{0}^{G G}\left(\alpha_{1}, \alpha_{2}\right) & =\widehat{K}_{0}^{G G}\left(\alpha_{1}, \alpha_{2}\right)-12 C_{A} \alpha_{1} \alpha_{2} .
\end{aligned}
$$

Here $(-1)^{d_{B}}$ has to be changed into $-(-1)^{d_{B}}$ in Eq. $(\sqrt[6.56]{)})$.

The anomalous dimensions for the polarized case have been derived for the first time in our previous paper [13] and were later confirmed in Ref. [31]. Those for the unpolarized case have been found several years before in Refs. [28, 18] already. In Ref. [28] also the necessity of the symmetrization Eq. (6.30) has been pointed out. The kernels $\widehat{K}^{A B}$ and $\Delta \widehat{K}^{A B}$ determine the respective evolutions of the operators $O_{(5)}^{\mathrm{NS}}, O_{(5)}^{q}$, and $O_{(5)}^{G}$ according to Eq. (6.28) in $\mathcal{O}\left(\alpha_{s}\right)$.

As we have seen above the $w$-representation reveals important properties of the kernels. It has been proven as a general representation in [5]. The connection between the $w$-variables and

\footnotetext{
${ }^{12}$ Note that the emergence of the term containing $\beta_{0}$ is due to self-energy graphs only and does not induce scale breaking effects at this order, 30.
} 
the $\alpha$-variables are given by Eqs. (6.17). Using these relations we get the following expressions for the anomalous dimensions:

$$
\begin{aligned}
K_{(1)}^{A B}\left(w_{1}, w_{2}, \kappa_{-}\right) & =\frac{\alpha_{s}\left(\mu^{2}\right)}{4 \pi}\left(\kappa_{-}\right)^{d_{B}-d_{A}}\left(\Theta\left(1-w_{2}-w_{1}\right) \Theta\left(1-w_{2}+w_{1}\right) \Theta\left(w_{2}\right) \widetilde{K}_{0}^{A B}\left(w_{1}, w_{2}\right)\right. \\
& \left.+(-1)^{d_{B}} \Theta\left(1+w_{2}-w_{1}\right) \Theta\left(1+w_{2}+w_{1}\right) \Theta\left(-w_{2}\right) \widetilde{K}_{0}^{A B}\left(w_{1},-w_{2}\right)\right) .
\end{aligned}
$$

For the unsymmetrized anomalous dimension kernels in leading order we obtain:

1) Unpolarized anomalous dimensions

$$
\begin{aligned}
\widetilde{K}_{0}^{q q}\left(w_{1}, w_{2}\right)= & C_{F}\left\{1-2 \delta\left(1-w_{2}+w_{1}\right)\left(1-\frac{2}{\left(1-w_{2}-w_{1}\right)_{+}}\right)\right. \\
& \left.\quad-2 \delta\left(1-w_{2}-w_{1}\right)\left(1-\frac{2}{\left(1-w_{2}+w_{1}\right)_{+}}\right)\right\}+3 C_{F} \delta\left(1-w_{2}\right) \delta\left(w_{1}\right) \\
\widetilde{K}_{0}^{q G}\left(w_{1}, w_{2}\right)= & -2 N_{f} T_{R}\left\{w_{2}+\left(1-w_{2}\right)^{2}-w_{1}^{2}\right\} \\
\widetilde{K}_{0}^{G q}\left(w_{1}, w_{2}\right)= & -2 C_{F}\left\{\delta\left(1-w_{2}\right) \delta\left(w_{1}\right)+1\right\} \\
\widetilde{K}_{0}^{G G}\left(w_{1}, w_{2}\right)= & C_{A}\left\{4 w_{2}+3\left(\left(1-w_{2}\right)^{2}-w_{1}^{2}\right)-2 \delta\left(1-w_{2}+w_{1}\right)\left(1+w_{2}-\frac{2}{\left(1-w_{2}-w_{1}\right)_{+}}\right)\right. \\
& \left.\quad-2 \delta\left(1-w_{2}-w_{1}\right)\left(1+w_{2}-\frac{2}{\left(1-w_{2}+w_{1}\right)_{+}}\right)\right\}+\beta_{0} \delta\left(1-w_{2}\right) \delta\left(w_{1}\right) .(6.70)
\end{aligned}
$$

2) Polarized anomalous dimensions

$$
\begin{aligned}
\Delta \widetilde{K}_{0}^{q q}\left(w_{1}, w_{2}\right) & =\widetilde{K}_{0}^{q q}\left(w_{1}, w_{2}\right) \\
\Delta \widetilde{K}_{0}^{q G}\left(w_{1}, w_{2}\right) & =-2 N_{f} T_{R}\left\{w_{2}\right\} \\
\Delta \widetilde{K}_{0}^{G q}\left(w_{1}, w_{2}\right) & =-2 C_{F}\left\{\delta\left(1-w_{2}\right) \delta\left(w_{1}\right)-1\right\} \\
\Delta \widetilde{K}_{0}^{G G}\left(w_{1}, w_{2}\right) & =\widetilde{K}_{0}^{G G}\left(w_{1}, w_{2}\right)+3 C_{A}\left(w_{1}^{2}-\left(1-w_{2}\right)^{2}\right) .
\end{aligned}
$$

Again the factor $(-1)^{d_{B}}$ in Eq. $(\overline{6.66})$ has to be changed into $-(-1)^{d_{B}}$.

Using Eqs. 6.48-6.51, 6.67-6.70), as well as Eqs. (6.62-6.65, 6.71-6.74) in the pseudoscalar case, we now derive the anomalous dimensions which are obtained in the local LCE directly from the non-local evolution kernels. Thereby the well-known relations

$$
\begin{aligned}
\int_{0}^{1} d w_{2} \frac{w_{2}^{n}}{\left(w_{2}-1\right)_{+}} & =\sum_{\ell=0}^{n-1} \frac{1}{\ell+1}=\psi(n+1)+\gamma_{E} \\
\int_{0}^{1} d w_{2} w_{2}^{a}\left(1-w_{2}\right)^{b} & =\frac{a ! b !}{(a+b+1) !}=B(a+1, b+1),
\end{aligned}
$$

are used. Here $\psi(x)=d \log (\Gamma(x)) / d x, \gamma_{E}$ denotes the Euler-Mascheroni constant, and $B(a, b)$ Euler's Beta-function. Again a common factor

$$
\frac{\alpha_{s}}{2 \pi} \times 2 \sigma_{n n^{\prime}}^{(-)} \quad \text { and } \quad \frac{\alpha_{s}}{2 \pi} \times 2 \sigma_{n n^{\prime}}^{(+)}
$$

in the unpolarized and polarized case will be suppressed, respectively. 
1) Unpolarized anomalous dimensions: ${ }^{13}$

$$
\begin{aligned}
\gamma_{n n^{\prime}}^{q q}= & C_{F}\left\{\left[\frac{1}{2}-\frac{1}{(n+1)(n+2)}+2 \sum_{j=2}^{n+1} \frac{1}{j}\right] \delta_{n n^{\prime}}-\left[\frac{1}{(n+1)(n+2)}+\frac{2}{n-n^{\prime}} \frac{n^{\prime}+1}{n+1}\right] \theta_{n n^{\prime}}\right\}(6.78) \\
\gamma_{n n^{\prime}}^{q G}= & -N_{f} T \frac{1}{(n+1)(n+2)(n+3)}\left[\left(n^{2}+3 n+4\right)-\left(n-n^{\prime}\right)(n+1)\right], \\
\gamma_{n n^{\prime}}^{G q}= & -C_{F} \frac{1}{n(n+1)(n+2)}\left[\left(n^{2}+3 n+4\right) \delta_{n n^{\prime}}+2 \theta_{n n^{\prime}}\right], \\
\gamma_{n n^{\prime}}^{G G}= & C_{A}\left\{\left[\frac{1}{6}-\frac{2}{n(n+1)}-\frac{2}{(n+2)(n+3)}+2 \sum_{j=2}^{n+1} \frac{1}{j}+\frac{2 N_{f} T}{3 C_{A}}\right] \delta_{n n^{\prime}}\right. \\
& \left.+\left[2\left(\frac{2 n+1}{n(n+1)}-\frac{1}{n-n^{\prime}}\right)-\left(n-n^{\prime}+2\right)\left(\frac{1}{n(n+1)}+\frac{1}{(n+2)(n+3)}\right)\right] \theta_{n n^{\prime}}\right\},
\end{aligned}
$$

with the following notation

$$
\begin{aligned}
\sigma_{n n^{\prime}}^{( \pm)} & =\frac{1}{4}\left(1 \pm(-1)^{n}\right)\left(1 \pm(-1)^{n^{\prime}}\right) \\
\theta_{n n^{\prime}} & = \begin{cases}1 & \text { for } n^{\prime}<n, \\
0 & \text { otherwise } .\end{cases}
\end{aligned}
$$

From this it becomes obvious that the symmetrization (6.26) and (6.27) of the anomalous dimension kernels only results into the property of the local anomalous dimension matrices being either odd-odd or even-even for the unpolarized or polarized case, respectively.

2) Polarized local anomalous dimensions:

$$
\begin{aligned}
\Delta \gamma_{n n^{\prime}}^{q q}= & \gamma_{n n^{\prime}}^{q q}, \\
\Delta \gamma_{n n^{\prime}}^{q G}= & -N_{f} T \frac{n^{\prime}}{(n+1)(n+2)}, \\
\Delta \gamma_{n n^{\prime}}^{G q}= & \frac{1}{(n+1)(n+2)}\left[(n+3) \delta_{n n^{\prime}}-\frac{2}{n} \theta_{n n^{\prime}}\right], \\
\Delta \gamma_{n n^{\prime}}^{G G}= & C_{A}\left\{\left[\frac{1}{6}-\frac{4}{(n+1)(n+2)}+2 \sum_{j=2}^{n+1} \frac{1}{j}+\frac{2 N_{f} T}{3 C_{A}}\right] \delta_{n n^{\prime}}\right. \\
& \left.+\left[2\left(\frac{2 n+1}{n(n+1)}-\frac{1}{n-n^{\prime}}\right)-\left(n-n^{\prime}+2\right) \frac{2}{(n+1)(n+2)}\right] \theta_{n n^{\prime}}\right\} .
\end{aligned}
$$

As already noted in Section 6.1 the well-known forward anomalous dimensions are obtained as the diagonal elements of Eqs. (6.78-6.81, 6.82-6.85).

The explicit computation of the anomalous dimensions is straightforward. In lowest order we have to determine the one-particle irreducible one-loop Feynman diagrams containing the considered operators as the first vertex. The new Feynman rules and an example of a calculation in the covariant gauge in the unpolarized case has been presented in [28]. Here we have performed the calculation in axial gauge which leads to essential simplifications in this order of perturbation theory. A sample calculation is given in Appendix B.

\footnotetext{
${ }^{13}$ In comparing these expressions with the values given in the literature 32, 33 the above mentioned shift of $\left(n, n^{\prime}\right)$ into $\left(n-1, n^{\prime}-1\right)$ sometimes has to be taken into account.
} 


\section{$7 \quad$ Evolution equations and evolution kernels}

The distribution amplitudes and partition functions defined in Section 4 have to be determined experimentally at a given factorization scale $\mu_{0}^{2}$. Their evolution in $\mu^{2}$, however, can be described within perturbative QCD and is ruled by renormalization group equations. In this Section we discuss generic evolution equations which directly follow from the renormalization group equation (6.9).

\subsection{General properties of twist-2 evolution equations}

Let us first discuss general properties of the evolution equations and their kernels at any order of perturbation theory. For simplicity we restrict the discussion to the non-singlet (NS) case since the arguments in the singlet case are quite analogous.

The evolution equations of the partition functions in question are consequences of the renormalization group equations of the complete twist-2 operators, i.e. including the trace terms which are proportional to $\tilde{x}_{\sigma}$. These operators are summed up local operators of definite twist. Despite the fact that the vector operator $O_{\sigma}^{\text {twist2 }}$ and the scalar operator $O^{\text {twist2 }}=\tilde{x}^{\sigma} O_{\sigma}^{\text {twist2 }}\left(\kappa_{1} \tilde{x}, \kappa_{2} \tilde{x}\right)$ fullfil the same renormalization group equation (6.1), as has been shown above, the evolution equations of their matrix elements are different.

For general values of the arguments $\kappa_{1}, \kappa_{2}$ the operator matrix elements read

$$
\left\langle p_{2}\left|O^{\mathrm{twist} 2}\left(\kappa_{1} x, \kappa_{2} x\right)\right| p_{1}\right\rangle=i \tilde{f}_{J}\left(\kappa_{-} x p_{+}, \kappa_{-} x p_{-}, \kappa_{-}^{2} x^{2}, p_{1} p_{2}, \mu^{2}\right) e^{i \kappa_{+} x p_{-}} \mathcal{Q}^{J}\left(x ; p_{2}, p_{1}\right)
$$

and

$$
\begin{array}{r}
\left\langle p_{2}\left|O^{\mu, \operatorname{twist} 2}\left(\kappa_{1} x, \kappa_{2} x\right)\right| p_{1}\right\rangle=i \mathcal{Q}^{J \mu}\left(p_{2}, p_{1}\right) \widetilde{F}_{J}\left(\kappa_{-} x p_{+}, \kappa_{-} x p_{-}, \kappa_{+} x p_{-}, \kappa_{-}^{2} x^{2}, p_{1} p_{2}, \mu^{2}\right) \\
+i \mathcal{Q}^{J}\left(x ; p_{2}, p_{1}\right) i\left\{\kappa_{-} p_{-}^{\mu} \partial_{\kappa_{-} x p_{-}}+\kappa_{+} p_{-}^{\mu} \partial_{\kappa_{+} x p_{-}}+\kappa_{-} p_{+}^{\mu} \partial_{\kappa_{+} x p_{-}}+2 x^{\mu} \kappa_{-}^{2} \partial_{\kappa_{-}^{2} x^{2}}\right\} \\
\times \widetilde{F}_{J},\left(\kappa_{-} x p_{+}, \kappa_{-} x p_{-}, \kappa_{-}^{2} x^{2}, p_{1} p_{2}, \mu^{2}\right)
\end{array}
$$

which is nothing but the Eq. (4.10) in the coordinate space.

Whereas for the representation of the Compton amplitude the operators and their matrix elements can be taken at arbitrary values of $x$, cf. Section 5 , for the renormalization properties considered in Section 6, it is essential that the operators are defined on the light-ray $\tilde{x}, \tilde{x}^{2}=0$. Furthermore, in the subsequent considerations the operators have to be taken on the light-ray at general values of $\kappa_{i}$.

The matrix elements of the scalar operators $O^{N S}$ obey the renormalization group equation

$$
\mu^{2} \frac{d}{d \mu^{2}}\left\langle p_{2}\left|O^{\mathrm{NS}}\left(\kappa_{1} \tilde{x}, \kappa_{2} \tilde{x}\right)\right| p_{1}\right\rangle=\int d^{2} \underline{\kappa}^{\prime} \gamma^{N S}\left(\kappa_{1}, \kappa_{2} ; \kappa_{1}^{\prime}, \kappa_{2}^{\prime}\right)\left\langle p_{2}\left|O^{\mathrm{NS}}\left(\kappa_{1}^{\prime} \tilde{x}, \kappa_{2}^{\prime} \tilde{x}\right)\right| p_{1}\right\rangle
$$

and correspondingly for $O_{5}^{N S}$ with $\gamma_{5}^{N S} \equiv \Delta \gamma^{N S}$. Having in mind the kinematic decomposition of the matrix elements, Eqs. (4.4, 7.1) we get renormalization group equations for the partition functions in coordinate space, $\tilde{f}_{J}$, separately:

$$
\mu^{2} \frac{d}{d \mu^{2}} \tilde{f}_{J}\left(\kappa_{-} \tilde{x} p_{+}, \kappa_{-} \tilde{x} p_{-}\right) e^{i \kappa_{+} \tilde{x} p_{-}}=\int d^{2} \underline{\kappa}^{\prime} \gamma^{N S}\left(\kappa_{1}, \kappa_{2} ; \kappa_{1}^{\prime}, \kappa_{2}^{\prime}\right) \tilde{f}_{J}\left(\kappa_{-}^{\prime} \tilde{x} p_{+}, \kappa_{-}^{\prime} \tilde{x} p_{-}\right) e^{i \kappa^{\prime}+\tilde{x} p_{-}} .
$$

In the same way we obtain the renormalization group equations for the matrix elements of the twist-2 vector operator $(\overline{7.2})$ and the corresponding partition functions $\widetilde{F}_{J}$. After the kinematic 
decomposition the operator relation Eq. (3.8) implies

$$
\mu^{2} \frac{d}{d \mu^{2}} \widetilde{F}_{J}\left(\kappa_{-} \tilde{x} p_{+}, \kappa_{-} \tilde{x} p_{-}, \kappa_{+} \tilde{x} p_{-}\right)=\int d^{2} \underline{\kappa}^{\prime} \gamma^{N S}\left(\kappa_{1}, \kappa_{2} ; \kappa_{1}^{\prime}, \kappa_{2}^{\prime}\right) \widetilde{F}_{J}\left(\kappa^{\prime} \tilde{x}_{p_{+}}, \kappa_{-}^{\prime} \tilde{x} p_{-}, \kappa_{+}^{\prime} \tilde{x} p_{-}\right) .
$$

By differentiation w.r.t. $\tilde{x} p_{-}$and $\tilde{x} p_{+}$the equations

$$
\begin{aligned}
\mu^{2} \frac{d}{d \mu^{2}}\left[\kappa_{-}^{\prime} \partial_{\kappa_{-}^{\prime} \tilde{x} p_{-}}+\kappa_{+}^{\prime} \partial_{\kappa_{+}^{\prime} \tilde{x} p_{-}}\right] \widetilde{F}_{J} & =\int d^{2} \underline{\kappa}^{\prime} \gamma^{N S}\left(\kappa_{1}, \kappa_{2} ; \kappa_{1}^{\prime}, \kappa_{2}^{\prime}\right)\left[\kappa_{-} \partial_{\kappa_{-} \tilde{x} p_{-}}+\kappa_{+} \partial_{\kappa_{+} \tilde{x} p_{-}}\right] \widetilde{F}_{J} \\
\mu^{2} \frac{d}{d \mu^{2}}\left[\kappa_{-} \partial_{\kappa_{-} \tilde{x} p_{+}}\right] \widetilde{F}_{J} & =\int d^{2} \underline{\kappa}^{\prime} \gamma^{N S}\left(\kappa_{1}, \kappa_{2} ; \kappa_{1}^{\prime}, \kappa_{2}^{\prime}\right)\left[\kappa_{-}^{\prime} \partial_{\kappa_{-}^{\prime} \tilde{x} p_{+}}\right] \widetilde{F}_{J}
\end{aligned}
$$

are obtained with the same arguments as in Eq. (7.5). The renormalization group equations are valid in the limit $x \rightarrow \tilde{x}$, i.e. $x^{2}=0$. Therefore a further relation

$$
\begin{aligned}
\mu^{2} \frac{d}{d \mu^{2}} & {\left[\kappa_{-}^{2} \partial_{\kappa_{-}^{2} x^{2}} \widetilde{F}\left(\kappa_{-} x p_{+}, \kappa_{-} x p_{-}, \kappa_{+} x p_{-}, \kappa_{-}^{2} x^{2}\right)\right]_{x \rightarrow \tilde{x}} } \\
& =\int d^{2} \underline{\kappa}^{\prime} \breve{\gamma}^{N S}\left(\kappa_{1}, \kappa_{2} ; \kappa_{1}^{\prime}, \kappa_{2}^{\prime}\right)\left[\kappa_{-}^{\prime 2} \partial_{\kappa_{-}^{\prime 2} x^{2}} \widetilde{F}\left(\kappa_{-}^{\prime} x p_{+}, \kappa_{-}^{\prime} x p_{-}, \kappa_{+}^{\prime} x p_{-}, \kappa_{-}^{\prime 2} x^{2}\right)\right]_{x \rightarrow \tilde{x}}
\end{aligned}
$$

holds. Here we will not discuss this special evolution equation in detail. We remark however that the corresponding anomalous dimension belongs to the operator

$$
O^{\prime}\left(\kappa_{1} \tilde{x}, \kappa_{2} \tilde{x}\right)=\left.\tilde{x}^{*} \frac{\partial}{\partial x} \frac{i}{2}\left[\bar{\psi}\left(\kappa_{1} x\right)(x \gamma) U\left(\kappa_{1}, \kappa_{2}\right) \psi\left(\kappa_{2} x\right)-\bar{\psi}\left(\kappa_{2} x\right)(x \gamma) U\left(\kappa_{1}, \kappa_{2}\right) \psi\left(\kappa_{1} x\right)\right]\right|_{x \rightarrow \tilde{x}}
$$

and has to be calculated independently. Here $\tilde{x}^{*}$ denotes a second independent light-like vector which obeys $\tilde{x}^{*} \tilde{x}=1$.

Note that there are two evolution equations, (7.4) and (7.5), for different partition functions which are non-trivially related by

$$
\widetilde{F}_{J}\left(\kappa_{-} \tilde{x} p_{+}, \kappa_{-} \tilde{x} p_{-}, \kappa_{+} \tilde{x} p_{-}\right)=\int_{0}^{1} d \lambda \tilde{f}_{J}\left(\lambda \kappa_{-} \tilde{x} p_{+}, \lambda \kappa_{-} \tilde{x} p_{-}\right) e^{i \lambda \kappa_{+} \tilde{x} p_{-}} .
$$

We finally show that this relation is compatible with applying the renormalization group operator. The evolution equation for $\widetilde{F}_{J}$ reads

$\mu^{2} \frac{d}{d \mu^{2}} \int_{0}^{1} d \lambda \tilde{f}_{J}\left(\lambda \kappa_{-} \tilde{x} p_{+}, \lambda \kappa_{-} \tilde{x} p_{-}\right) e^{i \lambda \kappa_{+} \tilde{x} p_{-}}=\int d^{2} \underline{\kappa}^{\prime} \int_{0}^{1} d \lambda \gamma^{\mathrm{NS}}\left(\lambda \kappa, \kappa^{\prime}\right) \tilde{f}_{J}\left(\kappa_{-}^{\prime} \tilde{x} p_{+}, \kappa_{-}^{\prime} \tilde{x} p_{-}\right) e^{i \kappa^{\prime}+\tilde{x} p_{-}}$

in the representation of Eq. (7.10). Changing the integration variables $\underline{\kappa^{\prime}}=\lambda \underline{\hat{\kappa}}$ and applying the scaling relation Eq. (6.11) for the anomalous dimensions

$$
\gamma(\lambda \underline{\kappa}, \lambda \underline{\hat{\kappa}})=\lambda^{-2} \gamma(\underline{\kappa}, \underline{\hat{\kappa}})
$$

we obtain

$\mu^{2} \frac{d}{d \mu^{2}} \int_{0}^{1} d \lambda f_{J}\left(\lambda \kappa_{-} \tilde{x} p_{+}, \lambda \kappa_{-} \tilde{x} p_{-}, \lambda \kappa_{+} \tilde{x} p_{-}\right)=\int d^{2} \underline{\hat{\kappa}} \gamma^{\mathrm{NS}}(\underline{\kappa}, \underline{\hat{\kappa}}) \int_{0}^{1} d \lambda f_{J}\left(\lambda \hat{\kappa}_{-} \tilde{x} p_{+}, \lambda \hat{\kappa}_{-} \tilde{x} p_{-}\right) e^{i \lambda \hat{\kappa}_{+} \tilde{x} p_{-}}$

which is the evolution equation (77.5). This shows that Eq. (7.5) and Eq. (7.4) are equivalent. The evolution equations (7.4-7.7) yield already a complete description in the non-singlet case. However, commonly the evolution of parton densities or distribution amplitudes is described in terms of functions which depend on the related momentum fractions. This description is obtained after a Fourier transformation of the above functions to the functions $f\left(z_{+}, z_{-}\right)$and $F\left(z_{+}, z_{-}\right)$which have been introduced in Section 4 before. In the following we will consider the evolution equations of these quantities. 


\subsection{Two-Variable Equations}

In this paragraph we derive the evolution equations for the double distribution amplitude in the singlet case. Let us start our consideration with the double-variable distributions $f_{J}^{q(2)}\left(z_{+}, z_{-}\right)$ and $f_{J}^{G(2)}\left(z_{+}, z_{-}\right)$. In accordance with Eqs. (4.4) and (4.22) we use the kinematic decompositions:

$$
\begin{aligned}
\kappa_{-}\left\langle p_{2}\right| O^{q, \text { twist2}}\left(-\kappa_{-} \tilde{x}, \kappa_{-} \tilde{x}\left|p_{1}\right\rangle\right. & =\left(\tilde{x} p_{+}\right)^{-1} \tilde{f}_{J}^{q(2)}\left(\kappa_{-} \tilde{x} p_{+}, \kappa_{-} \tilde{x} p_{-}, p_{1} p_{2}, \mu^{2}\right) \mathcal{Q}^{J}\left(\tilde{x} ; p_{2}, p_{1}\right), \\
\kappa_{-}^{2}\left\langle p_{2}\left|O^{G, \operatorname{twist} 2}\left(-\kappa_{-} \tilde{x}, \kappa_{-} \tilde{x}\right)\right| p_{1}\right\rangle & =\left(\tilde{x} p_{+}\right)^{-1} \tilde{f}_{J}^{G(2)}\left(\kappa_{-} \tilde{x} p_{+}, \kappa_{-} \tilde{x} p_{-}, p_{1} p_{2}, \mu^{2}\right) \mathcal{Q}^{J}\left(\tilde{x} ; p_{2}, p_{1}\right) .
\end{aligned}
$$

Combining both cases we use the notation $\tilde{f}^{A}$, where $A=q, G$, thereby suppressing the index (2). We are interested in the evolution equations for the physically relevant Fourier transforms

$$
f_{J}^{A}\left(z_{+}, z_{-}\right)=\int_{-\infty}^{\infty} \frac{d \kappa_{-} \tilde{x} p_{-}}{2 \pi} \int_{-\infty}^{\infty} \frac{d \kappa_{-} \tilde{x} p_{+}}{2 \pi} \tilde{f}_{J}^{A}\left(\kappa_{-} \tilde{x} p_{+}, \kappa_{-} \tilde{x} p_{-}\right) e^{i \kappa_{-} \tilde{x}\left(p_{+} z_{+}+p_{-} z_{-}\right)},
$$

where variables being irrelevant for the present considerations have been omitted. In order to get the evolution equations for the singlet case in $z$-space we have to perform a Fourier transform of the equations analogous to (7.4). We take matrix elements of the renormalization group equations (6.29) with the corresponding evolution kernels

$$
K^{A B}\left(w_{1}, w_{2}, \kappa_{-}\right)=\kappa_{-}^{d_{B}-d_{A}} \widetilde{K}^{A B}\left(w_{1}, w_{2}\right)
$$

and perform the Fourier transform to get the evolution equations for $f_{J}^{A}$. Inserting the definition (4.14) into the right hand side we obtain

$$
\begin{aligned}
\mu^{2} \frac{d}{d \mu^{2}} f_{J}^{A(2)}\left(z_{+}, z_{-}\right)=\int_{-\infty}^{\infty} \frac{d \kappa_{-} \tilde{x} p_{+}}{2 \pi} \int_{-\infty}^{\infty} \frac{d \kappa_{-} \tilde{x} p_{-}}{2 \pi} e^{i\left(\kappa_{+}^{\prime}-\kappa_{+}\right) \tilde{x} p_{-}} e^{i \kappa_{-} \tilde{x}\left(p_{+} z_{+}+p_{-} z_{-}\right)} \\
\quad \times \int D w K^{A B}\left(w_{1}, w_{2}, \kappa_{-}\right) \kappa_{-}^{d_{A}} \int D z^{\prime}\left(\kappa_{-}^{\prime}\right)^{-d_{B}} e^{-i \kappa_{-}^{\prime} \tilde{x}\left(p_{+} z_{+}^{\prime}+p_{-} z_{-}^{\prime}\right)} f_{J}^{B(2)}\left(z_{+}^{\prime}, z_{-}^{\prime}\right) .
\end{aligned}
$$

Carrying out the integration over $d\left(\kappa_{-} \tilde{x} p_{ \pm}\right)$we get

$$
\begin{aligned}
\mu^{2} \frac{d}{d \mu^{2}} f_{J}^{A(2)}\left(z_{+}, z_{-}\right)= & \int D w \widetilde{K}^{A B}\left(w_{1}, w_{2}\right) w_{2}^{-d_{B}} \\
& \times \int d z_{-}^{\prime} d z_{+}^{\prime} \delta\left(z_{-}-w_{2} z_{-}^{\prime}+w_{1}\right) \delta\left(z_{+}-w_{2} z_{+}^{\prime}\right) f_{J}^{B(2)}\left(z_{+}^{\prime}, z_{-}^{\prime}\right) .
\end{aligned}
$$

This finally leads to

$$
\begin{aligned}
\mu^{2} \frac{d}{d \mu^{2}} f_{J}^{A(2)}\left(z_{+}, z_{-}\right) & =\int D z^{\prime} \Gamma^{A B}\left(z_{+}, z_{-}, z_{+}^{\prime}, z_{-}^{\prime}\right) f_{J}^{B(2)}\left(z_{+}^{\prime}, z_{-}^{\prime}\right) \\
\Gamma^{A B}\left(z_{+}, z_{-}, z_{+}^{\prime}, z_{-}^{\prime}\right) & =\frac{1}{\left|z_{+}^{\prime}\right|} \widetilde{K}^{A B}\left(w_{1}=-z_{-}+\frac{z_{+}}{z_{+}^{\prime}} z_{-}^{\prime}, w_{2}=\frac{z_{+}}{z_{+}^{\prime}}\right)\left(\frac{z_{+}^{\prime}}{z_{+}}\right)^{d_{B}} .
\end{aligned}
$$

Now we consider the kinematic decomposition for the first parametrization:

$$
\begin{array}{rlr}
\left\langle p_{2}\left|O^{q, \text { twist2 }}\left(-\kappa_{-} \tilde{x}, \kappa_{-} \tilde{x}\right)\right| p_{1}\right\rangle & = & i \tilde{f}_{J}^{q(1)}\left(\kappa_{-} \tilde{x} p_{+}, \kappa_{-} \tilde{x} p_{-}, p_{1} p_{2}, \mu^{2}\right) \mathcal{Q}^{J}\left(\tilde{x} ; p_{2}, p_{1}\right) \\
\left\langle p_{2}\left|O^{G, \text { twist2 }}\left(-\kappa_{-} \tilde{x}, \kappa_{-} \tilde{x}\right)\right| p_{1}\right\rangle & =i\left(i \tilde{x} p_{+}\right) \tilde{f}_{J}^{G(1)}\left(\kappa_{-} \tilde{x} p_{+}, \kappa_{-} \tilde{x} p_{-}, p_{1} p_{2}, \mu^{2}\right) \mathcal{Q}^{J}\left(\tilde{x} ; p_{2}, p_{1}\right) .
\end{array}
$$

The derivation of the evolution equations for the distribution amplitudes Eqs. (7.20,7.21) follows the same line. The only difference is that the integration with respect to $\kappa_{-} \tilde{x} p_{+}$does not lead directly to the $\delta$-function and one obtains

$$
\mu^{2} \frac{d}{d \mu^{2}} f_{J}^{A}\left(z_{+}, z_{-}\right)=\int D z^{\prime} \Gamma^{A B(1)}\left(z_{+}, z_{-} ; z_{+}^{\prime}, z_{-}^{\prime}\right) f_{J}^{B}\left(z_{+}^{\prime}, z_{-}^{\prime}\right)
$$


with

$$
\Gamma^{A B(1)}\left(z_{+}, z_{-} ; z_{+}^{\prime}, z_{-}^{\prime}\right)=\int D w \widetilde{O}^{A B}\left(z_{+}-w_{2} z_{+}^{\prime}\right) \widetilde{K}^{A B}\left(w_{1}, w_{2}\right) \delta\left(z_{-}-w_{2} z_{-}^{\prime}+w_{1}\right),
$$

(no summation), where $\widetilde{O}^{A B}$ is given by

$$
\widetilde{O}^{A B}(z)=\frac{1}{2 \pi} \int \frac{d \kappa_{+} \tilde{x} p_{+}}{\left(i \kappa_{-} \tilde{x} p_{+}\right)^{d_{B}-d_{A}}} e^{i \kappa_{-} \tilde{x} p_{+} z}=\left\{\begin{array}{ccc}
\delta(z) & \text { for } & d_{B}-d_{A}=0 \\
\partial_{z} \delta(z) & \text { for } & d_{B}-d_{A}=1 \\
\frac{1}{2} \varepsilon(z) & \text { for } & d_{B}-d_{A}=-1
\end{array}\right.
$$

Here $\varepsilon(z)$ denotes the sign-function, cf. Eqs. (8.15, D.4).

\subsection{One-Variable Equations}

The equations given above cover the most general case. These equations depend on two partition variables $z_{+}$and $z_{-}$. The question arises whether it is possible to express them in terms of singlevariable distributions imposing a one-parameter constraint. Having (physical) scale invariance in mind it is reasonable to introduce a kinematic condition by

$$
\tau=\frac{\tilde{x} p_{-}}{\tilde{x} p_{+}}
$$

under which the evolution equations are derived. This approach has been outlined in [5, 13]. It is possible to define the matrix elements formally by

$$
\begin{aligned}
\left.\frac{\left\langle p_{2}\left|O^{q}\left(-\kappa_{-} \tilde{x}, \kappa_{-} \tilde{x}\right)\right| p_{1}\right\rangle}{\left(i \tilde{x} p_{+}\right)}\right|_{\tilde{x} p_{-}=\tau \tilde{x} p_{+}} & =\int_{-\infty}^{+\infty} d t e^{-i \kappa_{-} \tilde{x} p_{+} t} \Phi_{q}(t, \tau), \\
\left.\frac{\left\langle p_{2}\left|O^{G}\left(-\kappa_{-} \tilde{x}, \kappa_{-} \tilde{x}\right)\right| p_{1}\right\rangle}{\left(i \tilde{x} p_{+}\right)^{2}}\right|_{\tilde{x} p_{-}=\tau \tilde{x} p_{+}} & =\int_{-\infty}^{+\infty} d t e^{-i \kappa_{-} \tilde{x} p_{+} t} t \Phi_{G}(t, \tau) .
\end{aligned}
$$

Here $\Phi(t, \tau)$ denotes, in the above sense, a partition function which in the limit $\tau \rightarrow-1$ tends to the standard Brodsky-Lepage wave function. Note that the parameter $\tau$ is related to $x$-space. This parameter may change into the purely kinematic variable $\eta=q p_{-} / q p_{+}$in some applications after a Fourier transform. In Eq. (5.29)

$$
\int D z \delta\left(z_{+}-t+\eta z_{-}\right) f_{J}^{A(1)}\left(z_{+}=t-\eta z_{-}, z_{-}\right)=\hat{f}_{J}^{A(1)}(t, \eta) t^{d_{A}-1}
$$

$\eta$ occurs naturally as a variable in momentum space. The reverse formula reads

$$
f_{J}^{A(1)}\left(z_{+}, z_{-}\right)=\frac{1}{(2 \pi)^{2}} \int_{-\infty}^{+\infty} d t \int_{-\infty}^{+\infty} d \eta \frac{\left|z_{-}\right|}{\eta^{2}} \exp \left\{i \frac{z_{-}\left(z_{+}-t\right)}{\eta}\right\} t^{d_{A}-1} \hat{f}_{J}^{A(1)}(t, \eta) .
$$

Eq. (7.29) has been derived by assuming, that the restrictions of the support are completely included into the definition of the functions $\hat{f}_{J}^{A(1)}(t, \eta)$. This can be achieved using the representation (8.15) for the Heaviside-functions and performing the $\operatorname{limits} \lim _{\varepsilon \rightarrow 0^{+}}$later on, cf. [34]. One may equally well consider the set of one-variable functions $\hat{f}_{J}^{A(1)}(t, \eta)$ instead of the two-

variable functions $f_{J}^{A(1)}\left(z_{+}, z_{-}\right)$. Although the Compton amplitude depends on the functions 
$F_{J}^{A(1)}\left(z_{-}, z_{+}\right)$our evolution equations for simplicity have been formulated with the help of the functions $f_{J}^{A(1)}\left(z_{-}, z_{+}\right)$. However, both are related by

$$
F_{J}^{A(1)}\left(z_{+}, z_{-}\right)=\int_{0}^{1} \frac{d \lambda}{\lambda^{2}} f_{J}^{A(1)}\left(\frac{z_{+}}{\lambda}, \frac{z_{-}}{\lambda}\right) \Theta\left(\lambda-\left|z_{+}\right|\right) \Theta\left(\lambda-\left|z_{-}\right|\right) .
$$

The relation for $f_{J}^{A(1)}$ corresponding to Eq. (7.28) reads

$$
\widehat{F}_{J}^{A(1)}(t, \eta) t^{d_{A}-1}=\int D z F_{J}^{A(1)}\left(z_{+}, z_{-}\right) \delta\left(z_{+}-t+\eta z_{-}\right),
$$

which yields

$$
\widehat{F}_{J}^{A(1)}(t, \eta)=\int_{0}^{1} \frac{d \lambda}{\lambda} \widehat{f}_{J}^{A(1)}\left(\frac{t}{\lambda}, \eta\right)\left(\frac{t}{\lambda}\right)^{d_{A}-1}
$$

We finally obtain the one-parameter evolution equations for the functions $f_{J}^{N S}$ and $f_{J}^{A(1)}$ :

$$
\begin{aligned}
\mu^{2} \frac{d}{d \mu^{2}} \widehat{f}_{J}^{\mathrm{NS}}(t, \tau) & =\int_{-\infty}^{+\infty} d t^{\prime} V_{\mathrm{ext}}^{\mathrm{NS}}\left(t, t^{\prime}, \tau\right) \widehat{f}_{J}^{\mathrm{NS}}\left(t^{\prime}, \tau\right), \\
\mu^{2} \frac{d}{d \mu^{2}} \widehat{f}_{J}^{A(1)}(t, \tau) & =\int_{-\infty}^{+\infty} d t^{\prime} V_{\mathrm{ext}}^{A B}\left(t, t^{\prime}, \tau\right) \widehat{f}_{J}^{B(1)}\left(t^{\prime}, \tau\right),
\end{aligned}
$$

with $\widehat{f}_{J}^{\mathrm{NS}}=\widehat{f}_{J}^{q_{i}}-\widehat{f}_{J}^{\bar{q}_{j}}$. The corresponding extended kernels $V_{\mathrm{ext}}^{A B}\left(t, t^{\prime}, \tau\right) \mathrm{read}$

$$
\begin{aligned}
V_{\mathrm{ext}}^{A B}\left(t, t^{\prime}, \tau\right) & =\int_{0}^{1} d \alpha_{1} \int_{0}^{1-\alpha_{1}} d \alpha_{2} \widehat{K}^{A B}\left(\alpha_{1}, \alpha_{2}\right) \int_{-\infty}^{+\infty} \frac{d\left(\kappa \tilde{x} p_{+}\right)}{2 \pi} \\
& \times\left(i \kappa \tilde{x} p_{+}\right)^{d_{B}-d_{A}} \frac{t^{\prime d_{B}}}{t^{d_{A}}} \exp \left\{i \kappa \tilde{x} p_{+}\left[t-\left(1-\alpha_{1}-\alpha_{2}\right) t^{\prime}+\tau\left(\alpha_{1}-\alpha_{2}\right)\right]\right\} .
\end{aligned}
$$

They obey the scaling relation

$$
V_{\mathrm{ext}}^{A B}\left(t, t^{\prime}, \tau\right)=V_{\mathrm{ext}}^{A B}\left(t, t^{\prime},-\tau\right)=\frac{1}{\tau} V_{\mathrm{ext}}^{A B}\left(\frac{t}{\tau}, \frac{t^{\prime}}{\tau}, 1\right) .
$$

For convenience we rewrite the general expressions for the evolution kernels in the variables

$$
x=\frac{1}{2}\left(1+\frac{t}{\tau}\right), \bar{x}=\frac{1}{2}\left(1-\frac{t}{\tau}\right), y=\frac{1}{2}\left(1+\frac{t^{\prime}}{\tau}\right), \bar{y}=\frac{1}{2}\left(1-\frac{t^{\prime}}{\tau}\right) .
$$

In one-loop approximation they are given by

$$
V_{\mathrm{ext}}^{A B}\left(t, t^{\prime}, \tau\right)=\frac{\alpha_{s}\left(\mu^{2}\right)}{2 \pi} V_{0, \mathrm{ext}}^{A B}\left(t, t^{\prime}, \tau\right)
$$

where

$$
\begin{aligned}
V_{0, \mathrm{ext}}^{q q}\left(t, t^{\prime}, \tau\right) & =\frac{1}{2 \tau}\left\{V^{q q}(x, y) \rho(x, y)+V^{q q}(\bar{x}, \bar{y}) \rho(\bar{x}, \bar{y})+\frac{3}{2} C_{F} \delta(x-y)\right\} \\
V_{0, \mathrm{ext}}^{q G}\left(t, t^{\prime}, \tau\right) & =\frac{1}{2 \tau}\left(\frac{2 y-1}{2}\right)\left\{V^{q G}(x, y) \rho(x, y)-V^{q G}(\bar{x}, \bar{y}) \rho(\bar{x}, \bar{y})\right\} \\
V_{0, \mathrm{ext}}^{G q}\left(t, t^{\prime}, \tau\right) & =\frac{1}{2 \tau}\left(\frac{2}{2 x-1}\right)\left\{V^{G q}(x, y) \rho(x, y)-V^{G q}(\bar{x}, \bar{y}) \rho(\bar{x}, \bar{y})\right\}
\end{aligned}
$$




$$
\begin{aligned}
V_{0, \mathrm{ext}}^{G G}\left(t, t^{\prime}, \tau\right)= & \frac{1}{2 \tau}\left(\frac{2 y-1}{2 x-1}\right)\left\{V^{G G}(x, y) \rho(x, y)+V^{G G}(\bar{x}, \bar{y}) \rho(\bar{x}, \bar{y})\right\} \\
& +\frac{1}{2 \tau} \frac{1}{2} \beta_{0} \delta(x-y) \\
\Delta V_{0, \mathrm{ext}}^{q q}\left(t, t^{\prime}, \tau\right)= & V_{\mathrm{ext}}^{q q}\left(t, t^{\prime}, \tau\right) \\
\Delta V_{0, \mathrm{ext}}^{q G}\left(t, t^{\prime}, \tau\right)= & \frac{1}{2 \tau}\left(\frac{2 y-1}{2}\right)\left\{\Delta V^{q G}(x, y) \rho(x, y)-\Delta V^{q G}(\bar{x}, \bar{y}) \rho(\bar{x}, \bar{y})\right\} \\
\Delta V_{0, \mathrm{ext}}^{G q}\left(t, t^{\prime}, \tau\right)= & \frac{1}{2 \tau}\left(\frac{2}{2 x-1}\right)\left\{\Delta V^{G q}(x, y) \rho(x, y)-\Delta V^{G q}(\bar{x}, \bar{y}) \rho(\bar{x}, \bar{y})\right\} \\
\Delta V_{0, \mathrm{ext}}^{G G}\left(t, t^{\prime}, \tau\right)= & \frac{1}{2 \tau}\left(\frac{2 y-1}{2 x-1}\right)\left\{\Delta V^{G G}(x, y) \rho(x, y)+\Delta V^{G G}(\bar{x}, \bar{y}) \rho(\bar{x}, \bar{y})\right\} \\
& +\frac{1}{2 \tau} \frac{1}{2} \beta_{0} \delta(x-y)
\end{aligned}
$$

with the $\Theta$-structure

$$
\rho(x, y)=\Theta\left(1-\frac{x}{y}\right) \Theta\left(\frac{x}{y}\right) \operatorname{sign}(y),
$$

The non-singlet kernels are given by $V_{0, \text { ext }}^{\mathrm{NS}}=V_{0 \text {,ext }}^{q q}$ and $\Delta V_{0 \text {,ext }}^{\mathrm{NS}}=\Delta V_{0 \text {,ext }}^{q q}$, respectively. The different partial expressions $V^{A B}$ and $\Delta V^{A B}$ read

$$
\begin{aligned}
V^{q q}(x, y) & =C_{F}\left[\frac{x}{y}-\frac{1}{y}+\frac{1}{(y-x)_{+}}\right] \\
V^{q G}(x, y) & =-2 N_{f} T_{R} \frac{x}{y}\left[4(1-x)+\frac{1-2 x}{y}\right] \\
V^{G q}(x, y) & =C_{F}\left[1-\frac{x^{2}}{y}\right] \\
V^{G G}(x, y) & =C_{A}\left[2 \frac{x^{2}}{y}\left(3-2 x+\frac{1-x}{y}\right)+\frac{1}{(y-x)_{+}}-\frac{y+x}{y^{2}}\right] \\
\Delta V^{q q}(x, y) & =V^{q q}(x, y) \\
\Delta V^{q G}(x, y) & =-2 N_{f} T_{R} \frac{x}{y^{2}} \\
\Delta V^{G q}(x, y) & =C_{F}\left[\frac{x^{2}}{y}\right] \\
\Delta V^{G G}(x, y) & =C_{A}\left[2 \frac{x^{2}}{y^{2}}+\frac{1}{(y-x)_{+}}-\frac{y+x}{y^{2}}\right]
\end{aligned}
$$

Details on the []$_{+}-$prescription, which acts to the right, are given in Appendix 9 . Note that the kernels given in Eqs. (7.38-7.45) apply to the full range of variables, i.e. they represent the kernels completely. The function $V_{\text {ext }}^{q q}\left(t, t^{\prime}, \tau\right)$ was already derived in Refs. [35, 5].

The determination of these kernels is straightforward observing, however, that these quantities are distribution-valued. In the calculation one may set $\tau=1$ and later apply the scaling relation, Eq. (5.11), taking into account the general $\Theta$-structure in the $\left(t, t^{\prime}\right)$-plane derived in [5]. Alternatively, the calculation can be done in the different kinematic regions separately. A sample-calculation for the kernel $V^{G q}$ is given in Appendix D. All other known representations in the literature consist explicitly of two or three parts being calculated separately. 
Recently, the kernels (7.38-7.41) were also calculated in Ref. [11], using a different notation, cf. [36] for related work. For $\tau=1$, corresponding to $\zeta=1$ in [11], they agree with the results obtained above. A further independent representation has been given recently in Ref. [37]. The parameter $\tau$ introduced above equals to $-\xi / 2$ in the notation of [37.

\section{Special cases}

The evolution kernels given above cover a series of limiting cases which were studied in the literature before. These are characterized by special kinematic constraints for the processes, as in the case of forward scattering $\left\langle p_{2}\right| \rightarrow\left\langle p_{1}\right| \equiv\langle p|$ or the transition from the vacuum state $\langle 0|$ to a hadron state $\langle p|$ being related to the hadron wave functions.

\subsection{The Brodsky-Lepage limit}

For $\tau= \pm 1$ the equations (7.38-7.45) transform into the limit $\left\langle p_{2}\right| \rightarrow\langle p|,\left\langle p_{1}\right| \rightarrow\langle 0|$, which is known as the Brodsky-Lepage [38] and Efremov-Radyushkin [39] case. T4 This limit may be performed formally leaving $p_{1} \rightarrow 0$, which leads to correct results, cf. [35]. The corresponding evolution equations are obtained using as variables $x$ and $y$, Eqs. (7.36, 7.32, 7.33). As an example we consider the simplest case. For $0<x, y<1,|\tau|=1$ one obtains

$$
\begin{aligned}
V^{q q}\left(t, t^{\prime}\right)= & \frac{1}{2} C_{F}\left\{\Theta(y-x)\left[\frac{x}{y}-\frac{1}{y}+\frac{1}{(y-x)_{+}}\right]+\Theta(x-y)\left[\frac{1-x}{1-y}-\frac{1}{1-y}+\frac{1}{(x-y)_{+}}\right]\right. \\
& \left.+\frac{3}{2} \delta(x-y)\right\} .
\end{aligned}
$$

\subsection{The 'near-forward' representation}

This representation has been introduced by X. Ji and has also been given in Ref. [11]. It contains the forward case as limiting case. A correct application of the evolution equation for near forward matrix elements needs the representation for the region $t<\tau$ and $t>\tau$ separately. They are obtained from our general kernels as special cases. As an example we show here that our general structure for $V_{\text {ext }}^{q q}$ covers also the case $t>\tau, t^{\prime}>\tau$. Note that

$$
\operatorname{sign} \bar{y}=-\operatorname{sign} y \text { and } \Theta(1-\bar{x} / \bar{y})=\Theta(y-x)
$$

One therefore obtains

$$
\begin{aligned}
V_{0, \mathrm{ext}}^{q q}\left(t, t^{\prime}, \tau\right) & =\frac{1}{2} C_{F} \Theta(y-x)\left\{\frac{x}{y}\left[1+\frac{1}{(y-x)_{+}}\right]-\frac{1-x}{1-y}\left[1+\frac{1}{(x-y)_{+}}\right]\right\}+\frac{3}{2} C_{F} \delta(x-y) \\
& =\frac{1}{2} C_{F} \Theta(y-x) \frac{1}{(y-x)_{+}}\left[1+\frac{x \bar{x}}{y \bar{y}}\right]+\frac{3}{4} C_{F} \delta(x-y)
\end{aligned}
$$

for $\tau=1$. This representation was also given in Ref. [11].

\footnotetext{
${ }^{14}$ Several independent calculations of the evolution kernels for the meson wave functions were performed in Refs. 40.
} 
Starting from the anomalous dimension we reproduce the results of [37 and generalize them even to the region with $t^{\prime} \neq 1$. We obtain $\square$ :

$$
\begin{aligned}
K^{q q}\left(t, t^{\prime}, \xi\right)= & C_{F} \frac{t^{2}+t^{\prime 2}-\xi^{2} / 2}{\left(t^{2}-\xi^{2} / 4\right)\left(t^{\prime}-t\right)_{+}}+\frac{3}{2} \delta\left(t^{\prime}-t\right) \\
K^{q G}\left(t, t^{\prime}, \xi\right)= & T_{R} N_{f} \frac{t^{2}+\left(t^{\prime}-t\right)^{2}-\xi^{2} / 4}{\left(t^{\prime 2}-\xi^{2} / 4\right)^{2}} t^{\prime} \\
K^{G q}\left(t, t^{\prime}, \xi\right)= & C_{F} \frac{t^{\prime 2}+\left(t^{\prime}-t\right)^{2}-\xi^{2} / 4}{\left.t t^{\prime 2}-\xi^{2} / 4\right)} \\
K^{G G}\left(t, t^{\prime}, \xi\right)= & 2 C_{A} \frac{t^{\prime}}{t} \frac{1}{\left(t^{\prime 2}-\xi^{2} / 4\right)^{2}}\left[\frac{\left(t^{\prime 2}-\xi^{2} / 4\right)^{2}}{\left(t^{\prime}-t\right)_{+}}+t^{\prime}\left(t^{\prime 2}+\xi^{2} / 4\right)\right. \\
& \left.-t\left(3 t^{\prime 2}-\xi^{2} / 4\right)-\left(t^{\prime}+t\right)\left(t^{\prime}-t\right)^{2}\right]+\frac{\beta_{0}}{2} \delta\left(t^{\prime}-t\right), \\
\Delta K^{q q}\left(t, t^{\prime}, \xi\right)= & K^{q q}\left(t, t^{\prime}, \xi\right), \\
\Delta K^{q G}\left(t, t^{\prime}, \xi\right)= & T_{R} N_{f} \frac{t^{2}-\left(t^{\prime}-t\right)^{2}-\xi^{2} / 4}{\left(t^{\prime 2}-\xi^{2} / 4\right)^{2}} t^{\prime}, \\
\Delta K^{G q}(x, \xi)= & C_{F} \frac{t^{\prime}-\left(t^{\prime}-t\right)^{2}-\xi^{2} / 4}{t\left(t^{\prime 2}-\xi^{2} / 4\right)} \\
\Delta K^{G G}(x, \xi)= & 2 C_{A} \frac{t^{\prime}}{t} \frac{1}{\left(t^{\prime 2}-\xi^{2} / 4\right)^{2}}\left[\frac{\left(t^{\prime 2}-\xi^{2} / 4\right)^{2}}{\left(t^{\prime}-t\right)_{+}}+t^{\prime}\left(t^{\prime 2}+\xi^{2} / 4\right) .\right. \\
& \left.-t\left(3 t^{\prime 2}-\xi^{2} / 4\right)-2 t^{\prime}\left(t^{\prime}-t\right)^{2}\right]+\frac{\beta_{0}}{2} \delta\left(t^{\prime}-t\right)
\end{aligned}
$$

To get a complete representation of the kernels the independently calculated parts for $t<-\xi / 2$ and $|t|<\xi / 2$ have to be added, which can be directly derived from the general expressions given above.

\subsection{The Altarelli-Parisi limit}

Let us consider the case of forward scattering $p_{2}=p_{1} \equiv p$. The corresponding evolution kernels can be obtained after some calculation from Eqs. (7.38-7.45) in the limit $\tau \rightarrow 0, t^{\prime}>0$,

$$
\lim _{\tau \rightarrow 0} V_{0, e x t}^{A B}\left(t, t^{\prime}, \tau\right)=\frac{1}{t^{\prime}} P^{A B}(z) \Theta(z) \Theta(1-z) \quad \text { with } \quad z=\frac{t}{t^{\prime}},
$$

cf. Appendix D. Alternatively, the splitting functions for deeply inelastic forward scattering can be obtained by direct integration of the kernels Eqs. 6.57-6.65), which is performed in the following. The splitting functions are obtained by

$$
\begin{aligned}
& \widehat{P}^{A B}(z)=P^{A B}(z) \theta(z) \theta(1-z) \\
& P^{A B}(z)=\int_{-\infty}^{+\infty} d u \theta(1-u) \theta(u)(1-u) \widehat{O}^{A B}(u, z) \int_{0}^{1} d \xi \widehat{K}^{A B}((1-u) \xi,(1-u)(1-\xi))
\end{aligned}
$$

where

$$
\widehat{O}^{A B}(u, z)=\left(\begin{array}{cc}
\delta(z-u) & \partial_{z} \delta(z-u) \\
-\theta(z-u) / z & \delta(z-u) / z
\end{array}\right)
$$

\footnotetext{
${ }^{15}$ In ref. [37] the variable $t$ was denoted by $x$, having a different meaning in our notation. Therefore we use $t$ instead in Eqs. (8.4-8.11). Here our variable $\tau$ has to be identified with the variable $-\xi / 2$ from 37 .
} 
The $\Theta$-structure resulting from the measure $D \alpha$ is universal. In deriving Eq. (8.13) it is useful to apply the relations

$$
\theta(x)=\lim _{\varepsilon \rightarrow 0^{+}} \frac{1}{2 \pi} \int_{-\infty}^{+\infty} d \xi \frac{e^{i x \xi}}{i \xi+\varepsilon}, \quad \delta^{(k)}(x)=\frac{1}{2 \pi} \int_{-\infty}^{+\infty} d \xi(i \xi)^{k} e^{i x \xi},
$$

which are valid for tempered distributions [34]. $P^{A B}(z)\left(\triangle P^{A B}(z)\right)$ are the well-known splitting functions [32, 33] for unpolarized and polarized deep-inelastic forward scattering in $\mathcal{O}\left(\alpha_{s}\right)$ :

$$
\begin{aligned}
P^{q q}(z) & =C_{F}\left(\frac{1+z^{2}}{1-z}\right)_{+} \\
P^{q G}(z) & =2 N_{f} T_{R}\left[z^{2}+(1-z)^{2}\right] \\
P^{G q}(z) & =C_{F} \frac{1+(1-z)^{2}}{z} \\
P^{G G}(z) & =2 C_{A}\left[\frac{1}{z}+\frac{1}{(1-z)_{+}}-2+z(1-z)\right]+\frac{\beta_{0}}{2} \delta(1-z), \\
\Delta P^{q q}(z) & =P^{q q}(z), \\
\Delta P^{q G}(z) & =2 N_{f} T_{R}\left[z^{2}-(1-z)^{2}\right], \\
\Delta P^{G q}(z) & =C_{F} \frac{1-(1-z)^{2}}{z}, \\
\Delta P^{G G}(z) & =2 C_{A}\left[1-2 z+\frac{1}{(1-z)}\right]+\frac{\beta_{0}}{2} \delta(1-z) .
\end{aligned}
$$

The parton densities of the quarks $q(z)$ and antiquarks $\bar{q}(z)$ are given in Eqs. (5.22, 5.23) above, with $z$ denoting the momentum fraction carried by the partons. $G(z)$ denotes the gluon distribution. Their evolution equations read in leading order for the unpolarized case

$$
\begin{aligned}
\mu^{2} \frac{d}{d \mu^{2}} q^{\mathrm{NS}}\left(z, \mu^{2}\right) & =\frac{\alpha_{s}\left(\mu^{2}\right)}{2 \pi} \int_{z}^{1} \frac{d z^{\prime}}{z^{\prime}} \widehat{P}^{\mathrm{NS}}\left(\frac{z}{z^{\prime}}\right) q^{\mathrm{NS}}\left(z^{\prime}, \mu^{2}\right), \\
\mu^{2} \frac{d}{d \mu^{2}}\left(\begin{array}{c}
\Sigma\left(z, \mu^{2}\right) \\
G\left(z, \mu^{2}\right)
\end{array}\right) & =\frac{\alpha_{s}\left(\mu^{2}\right)}{2 \pi} \int_{z}^{1} \frac{d z^{\prime}}{z^{\prime}} \widehat{\boldsymbol{P}}\left(\frac{z}{z^{\prime}}\right)\left(\begin{array}{c}
\Sigma\left(z^{\prime}, \mu^{2}\right) \\
G\left(z^{\prime}, \mu^{2}\right)
\end{array}\right) .
\end{aligned}
$$

Here $q^{\mathrm{NS}}\left(z, \mu^{2}\right)$ denotes a non-singlet combination of quark densities, as e.g. $q\left(z, \mu^{2}\right)-\bar{q}\left(z, \mu^{2}\right)$, $\Sigma\left(z, \mu^{2}\right)=\sum_{j=1}^{N_{f}}\left[q_{j}\left(z, \mu^{2}\right)+\bar{q}_{j}\left(z, \mu^{2}\right)\right]$ is the flavor-singlet combination of the quark densities, and $\widehat{\boldsymbol{P}}$ is the matrix of the leading order singlet splitting functions. The respective evolution equations in the polarized case are obtained by replacing $q, \bar{q}$ and $G$ by $\Delta q, \Delta \bar{q}$, and $\Delta G$ and the splitting functions $\widehat{P}^{A B}(z)$ by $\Delta \widehat{P}^{A B}(z)$.

\section{Solutions}

Here we extend the method used in Ref. [10] for the non-singlet case to the singlet case. The idea follows the original solution of the Brodsky-Lepage equation given in [39. We start from Eq. (7.17) and form the moments of the distribution functions

$$
f_{n}^{A(2)}\left(z_{-}\right)=\int_{-1+\left|z_{-}\right|}^{1-\left|z_{-}\right|} d z_{+} z_{+}^{n} f^{A(2)}\left(z_{+}, z_{-}\right) .
$$


leading to

$$
\mu^{2} \frac{d}{d \mu^{2}} f_{n}^{A(2)}\left(z_{-}\right)=\frac{\alpha_{s}\left(\mu^{2}\right)}{2 \pi} \int_{-1}^{+1} d z_{-}^{\prime} \Gamma_{n}^{A B}\left(z_{-}, z_{-}^{\prime}\right) f_{n}^{B(2)}\left(z_{-}^{\prime}\right),
$$

with

$$
\begin{aligned}
\Gamma_{n}^{A B}\left(z_{-}, z_{-}^{\prime}\right)= & \int D w \delta\left(z_{-}-w_{2} z_{-}^{\prime}+w_{1}\right) w_{2}^{n-d_{B}} \widetilde{K}_{0}^{A B}\left(w_{1}, w_{2}\right) \\
= & \frac{1}{2} \int_{0}^{1} d w_{2} \Theta\left(1+z_{-}-w_{2}\left(1+z_{-}^{\prime}\right)\right) \Theta\left(1-z_{-}-w_{2}\left(1-z_{-}^{\prime}\right)\right) \\
& \times \Theta\left(1-z_{-}^{\prime}\right) \Theta\left(1+z_{-}^{\prime}\right) \widetilde{K}_{0}^{A B}\left(-z_{-}+w_{2} z_{-}^{\prime}, w_{2}\right) w_{2}^{n-d_{B}} .
\end{aligned}
$$

In this way a first diagonalization is obtained. The kernels $\Gamma_{n}^{A B}\left(z_{-}, z_{-}^{\prime}\right)$ read in the unpolarized case

$$
\begin{aligned}
\Gamma_{n}^{q q}\left(z, z^{\prime}\right)=C_{F} & \left\{\Theta\left(z-z^{\prime}\right)\left(\frac{1-z}{1-z^{\prime}}\right)^{n}\left(\frac{1}{n}+\frac{2}{\left(z-z^{\prime}\right)_{+}}\right)\right. \\
& \left.+\Theta\left(z^{\prime}-z\right)\left(\frac{1+z}{1+z^{\prime}}\right)^{n}\left(\frac{1}{n}+\frac{2}{\left(z^{\prime}-z\right)_{+}}\right)\right\}+3 \delta\left(z-z^{\prime}\right), \\
\Gamma_{n}^{q G}\left(z, z^{\prime}\right)=- & -N_{f} T_{R}\left\{\Theta\left(z-z^{\prime}\right)\left(\frac{1-z}{1-z^{\prime}}\right)^{n} \frac{n^{2}+2 n\left(z-z^{\prime}\right)-\left(2 z z^{\prime}-1\right)}{(n+1) n(n-1)}\right. \\
& \left.+\Theta\left(z^{\prime}-z\right)\left(\frac{1+z}{1+z^{\prime}}\right)^{n} \frac{n^{2}+2 n\left(z^{\prime}-z\right)-\left(2 z z^{\prime}-1\right)}{(n+1) n(n-1)}\right\}, \\
\Gamma_{n}^{G q}\left(z, z^{\prime}\right)=- & C_{F}\left\{\Theta\left(z-z^{\prime}\right)\left(\frac{1-z}{1-z^{\prime}}\right)^{n} \frac{1}{n}+\Theta\left(z^{\prime}-z\right)\left(\frac{1+z}{1+z^{\prime}}\right)^{n} \frac{1}{n}+\delta\left(z-z^{\prime}\right)\right\}, \\
\Gamma_{n}^{G G}\left(z, z^{\prime}\right)= & C_{A}\left\{\Theta ( z - z ^ { \prime } ) ( \frac { 1 - z } { 1 - z ^ { \prime } } ) ^ { n } \left(\frac{3(1+z)\left(1-z^{\prime}\right)}{n-1}+\frac{6 z z^{\prime}}{n}+\frac{3\left(1+z^{\prime}\right)(1-z)}{n+1}\right.\right. \\
& +\Theta\left(z^{\prime}-z\right)\left(\frac{1+z}{1+z^{\prime}}\right)^{n}\left(\frac{3(1-z)\left(1+z^{\prime}\right)}{n-1}+\frac{6 z z^{\prime}}{n}+\frac{3\left(1-z^{\prime}\right)(1+z)}{n+1}\right. \\
& \left.\left.-\frac{2}{n}+\frac{2}{\left(z^{\prime}-z\right)_{+}}\right)\right\}+\beta_{0} \delta\left(z-z^{\prime}\right) .
\end{aligned}
$$

Similarly one obtains for the kernels in the polarized case

$$
\begin{aligned}
& \Delta \Gamma_{n}^{q q}\left(z, z^{\prime}\right)=C_{F}\left\{\Theta\left(z-z^{\prime}\right)\left[\left(\frac{1-z}{1-z^{\prime}}\right)^{n}\left(\frac{1}{n}+\frac{2}{\left(z-z^{\prime}\right)_{+}}\right)\right]\right. \\
&+\left.+\Theta\left(z^{\prime}-z\right)\left[\left(\frac{1+z}{1+z^{\prime}}\right)^{n}\left(\frac{1}{n}+\frac{2}{\left(z^{\prime}-z\right)_{+}}\right)\right]+3 \delta\left(z-z^{\prime}\right)\right\} \\
& \Delta \Gamma_{n}^{q G}\left(z, z^{\prime}\right)=-2 N_{f} T_{R}\left\{\Theta\left(z-z^{\prime}\right)\left(\frac{1-z}{1-z^{\prime}}\right)^{n} \frac{1}{n}+\Theta\left(z^{\prime}-z\right)\left(\frac{1+z}{1+z^{\prime}}\right)^{n} \frac{1}{n}\right\} \\
& \Delta \Gamma_{n}^{G q}\left(z, z^{\prime}\right)=C_{F}\left\{\Theta\left(z-z^{\prime}\right)\left(\frac{1-z}{1-z^{\prime}}\right)^{n} \frac{1}{n}+\Theta\left(z^{\prime}-z\right)\left(\frac{1+z}{1+z^{\prime}}\right)^{n} \frac{1}{n}-\delta\left(z-z^{\prime}\right)\right\}, \\
& \Delta \Gamma_{n}^{G G}\left(z, z^{\prime}\right)=C_{A}\left\{\Theta\left(z-z^{\prime}\right)\left(\frac{1-z}{1-z^{\prime}}\right)^{n}\left(\frac{4}{n}+\frac{2}{\left(z-z^{\prime}\right)_{+}}\right)\right. \\
&\left.+\Theta\left(z^{\prime}-z\right)\left(\frac{1+z}{1+z^{\prime}}\right)^{n}\left(\frac{4}{n}+\frac{2}{\left(z^{\prime}-z\right)_{+}}\right)\right\}+\beta_{0} \delta\left(z-z^{\prime}\right) .
\end{aligned}
$$


As a next step we have to find a solution of Eq. (9.3). One finds that the following symmetryrelations hold for kernels $\Gamma_{n}^{G G}\left(z, z^{\prime}\right)$ and $\Delta \Gamma_{n}^{G G}\left(z, z^{\prime}\right)$ :

$$
\begin{aligned}
\left(1-z^{\prime 2}\right)^{n} \Gamma_{n}^{A B}\left(z, z^{\prime}\right) & =\Gamma_{n}^{A B}\left(z^{\prime}, z\right)\left(1-z^{2}\right)^{n} \\
\left(1-z^{\prime 2}\right)^{n} \Delta \Gamma_{n}^{A B}\left(z, z^{\prime}\right) & =\Delta \Gamma_{n}^{A B}\left(z^{\prime}, z\right)\left(1-z^{2}\right)^{n} .
\end{aligned}
$$

This is expected because of similar problems studied previously in [10, 39]. The final diagonalization of Eq. (9.2), referring to the partition functions $f_{n}^{A(2)}\left(z_{-}\right)$for which the quarkonic and gluonic operators are dealt with equally, can thus be performed using Gegenbauer polynomials $C_{m}^{p}(z)$, cf. [41],

$$
\begin{aligned}
\Gamma_{n}^{A B}\left(z, z^{\prime}\right) & =\sum \Gamma_{n, m}^{A B}\left(1-z^{2}\right)^{n} C_{m}^{n+1 / 2}(z) C_{m}^{n+1 / 2}\left(z^{\prime}\right) N_{n, m}^{-1}, \\
\Delta \Gamma_{n}^{A B}\left(z, z^{\prime}\right) & =\sum \Delta \Gamma_{n, m}^{A B}\left(1-z^{2}\right)^{n} C_{m}^{n+1 / 2}(z) C_{m}^{n+1 / 2}\left(z^{\prime}\right) N_{n, m}^{-1} .
\end{aligned}
$$

The coefficients $N_{n, m}$ are the normalization factors of the Gegenbauer polynomials

$$
\int_{-1}^{+1} d z\left(1-z^{2}\right)^{n} C_{l}^{n+1 / 2}(z) C_{k}^{n+1 / 2}(z)=\delta_{l k} N_{n, k}=\delta_{l k}\left[2^{2 n+1} \Gamma^{2}\left(n+\frac{1}{2}\right) \frac{(k+n+1 / 2) \cdot k !}{2 \pi \Gamma(2 n+1+k)}\right]^{-1} .
$$

$\Gamma_{n, m}^{A B}$ and $\Delta \Gamma_{n, m}^{A B}$ are the respective expansion coefficients which can be easily calculated and are found to be the diagonal elements of the triangular matrices discussed in Section 6 .

Another method of solution consists in forming also Mellin moments in the variable $z_{-}$,

$$
\int_{-1}^{+1} d z_{-} z_{-}^{k} f_{n}^{A(2)}\left(z_{-}\right)=f_{n, k}^{A(2)}
$$

The evolution equations may be written as, cf. also [28],

$$
\mu^{2} \frac{d}{d \mu^{2}} f_{n k}^{A(2)}=\frac{\alpha_{s}\left(\mu^{2}\right)}{2 \pi} \sum_{l=0}^{k} \Gamma_{n, k l}^{A B} f_{n l}^{A(2)}
$$

with the transformed kernels given by

$$
\Gamma_{n, k l}^{A B}=\int D w_{+} w_{2}^{n+l-d_{B}} w_{1}^{k-l} \widetilde{K}_{0}^{A B}\left(w_{1}, w_{2}\right)\left(\begin{array}{c}
k \\
l
\end{array}\right)
$$

These evolution equations are not diagonal with respect to the indices $(k, l)$. The explicit expressions for $\Gamma_{n, k l}^{A B}$ and $\Delta \Gamma_{n, k l}^{A B}$ are given in Eqs. (6.78-6.81, 6.82-6.85). For fixed $n$ they form triangular matrices. The eigenvalues are the diagonal elements $k=l$

$$
\Gamma_{n, k k}^{A B}=\int D w_{+} w_{2}^{n+k-d_{B}} \widetilde{K}_{0}^{A B}\left(w_{1}, w_{2}\right)=\gamma_{n+k-d_{B}}^{A B} .
$$

The coefficients $\gamma_{n+k-h_{j}}^{A B}$ are the anomalous dimensions of the forward case with a shifted Mellin index. A solution for the singlet evolution equations in the case of the single-variable equations was given in 42 recently. 


\section{Conclusions}

The scaling violations of the various observables, which occur in space-like processes, can be traced back to the renormalization of light-ray quark and gluon operators, which define these quantities. Therefore one may obtain a general description by calculating first the anomalous dimensions, or evolution kernels, of these operators and establish their connection to the different observables through the respective integral mappings and, in some cases, subsequent limiting procedures, afterwards. In the present paper we were limiting the investigation to the case of twist-2 operators and massless QCD. They are found by a corresponding twist-decomposition starting with a genuine operator representation. The space-like evolution kernels are processindependent. To form the observables one has to calculate also the process-dependent coefficient functions, which are trivial in lowest order. Furthermore, expectation values of the operators have to be formed, allowing for the description of a wide variety of processes.

In the light-cone expansion both vector operators $O^{\sigma}$ and scalar operators $\tilde{x}_{\sigma} O^{\sigma}$ appear. As was shown they are equivalent under renormalization and it suffices therefore to study the evolution of the simpler scalar operators. The respective relations for the vector operators can be obtained by integral relations. This holds also for the relation between their general non-forward expectation values between the states $\left\langle p_{2}\right|$ and $\left|p_{1}\right\rangle$. This connection is an important one, as it forms the basis for the integral relations by Wandzura and Wilczek [19] and one of the authors and Kochelev [20]. These relations were derived earlier in the local operator product expansion and a subsequent analytic continuation from the integer moments and a Mellin-transform. Here it is obtained directly by the integral relation between the scalar and vector operators.

One may derive a series of all-order relations for the evolution kernels of the light-ray operators. In the local representation they are given by infinite triangular matrices $\gamma_{n_{1}, n_{2}}^{A B}$, the diagonal elements of which are the anomalous dimensions in the forward scattering case. The anomalous dimensions are independent of the the total spin $n_{1}+n_{2}$.

The expectation values of the operators between the states $\left\langle p_{2}\right|$ and $\left|p_{1}\right\rangle$ are complex-valued distribution amplitudes through which the scattering cross section may be represented, which are, however, no observables. Nonetheless they obey evolution equations which are implied by the renormalization group equations of the operators. Commonly these matrix elements are represented by a two-fold Fourier transform as distribution amplitudes which depend on the two momentum fractions $z_{+}$and $z_{-}$along the momenta $p_{+}$and $p_{-}$. This representation is a generalization of the case of forward scattering in which only one momentum fraction emerges.

The two-variable amplitudes may be related to single-variable amplitudes by implying a kinematic constraint to the general case, i.e. demanding $\tau=\tilde{x} p_{-} / \tilde{x} p_{+}=$const. In this representation the kernels $V_{\text {ext }}^{A B}$ depend on the variables $t$ and $t^{\prime}$ besides the parameter $\tau$. We obtained a unique representation for the whole kinematic range of $t$ and $t^{\prime}$. One may easily describe the scaling violations in the case of vacuum-meson transitions [38, 39] in the limit $\tau \rightarrow-1$. With the help of the functions $V_{\text {ext }}^{A B}\left(t, t^{\prime}, \tau\right)$ other recent representations [11, 37] can be verified after some calculation. The evolution kernels in the limit of forward scattering, which are the well-known splitting functions, can be obtained by an integral relation form the two-variable kernels at one hand. They result also from the single-variable representation in the limit $\tau \rightarrow 0$ for $t^{\prime}>0$ from the kernels $V_{\text {ext }}^{A B}\left(t, t^{\prime}, \tau\right)$.

The solution of the two-variable non-singlet and singlet evolution equations require a diagonalization of the evolution kernels with respect to the variables $z_{+}, z_{+}^{\prime}$ and $z_{-}, z_{-}^{\prime}$, respectively. Here the $w$-representation shows, that the diagonalization with respect to the first variables can be performed by a Mellin transform, as in the forward case. This is an all-order property. The 
diagonalization with respect to the second set of variables through Gegenbauer polynomials relies on a symmetry property of the kernels, which exists at leading order, but is broken in higher orders. Alternatively, one may perform a second Mellin transform. The latter representation is, however, only suited for discrete representations over integer moments. An easy analytic continuation is lacking in the latter case since the eigenvectors do not obey an analytic relation which can be continued to complex numbers.

\section{Acknowledgement}

We would like to thank P. Söding and U. Gensch for their constant support of the project. For discussions we thank A. Tkabladze, W.L. van Neerven, D. Müller, A.V. Radyushkin, I.I. Balitsky, and W. Schweiger. We are very much indebted to T. Braunschweig for discussions on the relation between non-local and local anomalous dimensions, and to M. Lazar for various discussions on the twist decomposition of light-ray operators. B.G. would like to thank DESY Zeuthen and the Institute of Theoretical Physics at Graz University for support through the Scientific Exchange Program. D.R. likes to thank DESY Zeuthen, the Karl-Franzens-University of Graz, and the Graduate College 'Quantum Field Theory' at NTZ, Leipzig University, for the kind hospitality extended to him. We would like to thank J. Vermaseren for providing us a new version of AXODRAW. The work was supported in part by EU contract FMRX-CT98-0194(DG12-MIHT). 


\section{A Kinematic Relations}

The Compton process is characterized by the invariants

$$
\begin{aligned}
& s=\left(p_{1}+q_{1}\right)^{2}=\left(p_{2}+q_{2}\right)^{2}=M_{1}^{2}-Q_{1}^{2}+2 p_{1} q_{1}=M_{2}^{2}-Q_{2}^{2}+2 p_{2} q_{2} \\
& t=\left(p_{2}-p_{1}\right)^{2}=\left(q_{2}-q_{1}\right)^{2}=M_{1}^{2}+M_{2}^{2}-2 p_{1} p_{2}=-Q_{1}^{2}-Q_{2}^{2}-2 q_{1} q_{2} .
\end{aligned}
$$

Here $M_{1,2}$ are the masses of the initial- and final state hadron. $q_{1,2}^{2}=-Q_{1,2}^{2}$ are the virtualities of the incoming and outgoing photon. The initial-state photon is assumed to be space-like

$$
Q_{1}^{2}=-q_{1}^{2}>0
$$

while the final state photon can either be light- or time-like with

$$
q_{2}^{2}<m_{\pi}^{2}
$$

to avoid $s$-channel mass thresholds in the subsequent description, as they occur in reactions like $\gamma^{*}+p \rightarrow V+p^{\prime}$, where $p$ and $p^{\prime}$ are the initial and final-state hadrons, respectively, and $V$ denotes a meson at the photon-side. In the latter case the process is no longer space-like and the light cone expansion cannot be applied. On the other hand, one may produce mesons on the hadronic side in a space-like process.

The inelasticity $\nu$ is given by

$$
\nu=p_{+} q_{1}+\frac{1}{2}\left(M_{1}^{2}-M_{2}^{2}\right)=p_{+} q_{2}-\frac{1}{2}\left(M_{1}^{2}-M_{2}^{2}\right),
$$

and the cms-energy $s$ reads

$$
s=2 \nu+\frac{1}{2}\left[q_{1}^{2}+q_{2}^{2}+M_{1}^{2}+M_{2}^{2}-t\right] .
$$

The virtuality $Q^{2}=-q^{2}$ is

$$
Q^{2}=\frac{t}{4}+\frac{1}{2}\left(Q_{1}^{2}+Q_{2}^{2}\right)
$$

We define

$$
\hat{x}=\frac{Q_{1}^{2}}{\nu} .
$$

The variable $\xi$ is given by

$$
\xi=\frac{\hat{x}}{2}+\frac{t+2 Q_{2}^{2}}{4 \nu}=\hat{x}+\eta+\frac{t}{4 \nu}
$$

showing the relation of the variables $\xi$ and $\eta$. For $t / \nu \rightarrow 0$, as assumed in the generalized Bjorken region, their difference is $\hat{x}$. In the case of forward scattering, $\eta=t=0, \xi$ and $\hat{x}$ turn into the Bjorken variable $x_{B}=Q_{1}^{2} /\left(2 p \cdot q_{1}\right)$. Also other kinematic domains were studied for the Compton amplitude, as e.g. wide-angel scattering $p_{1} \cdot p_{2} \rightarrow \infty$ [43, a quantity being fixed in the present treatment. 


\section{B Sample calculation of the anomalous dimensions}

In this Appendix a sample calculation for the non-local anomalous dimensions is performed. As an example we will consider the derivation of the anomalous dimension $\Delta K^{G q}$. The calculation is performed in the $\overline{\mathrm{MS}}$-scheme using dimensional regularization in $N=4-2 \varepsilon$ dimensions. The anomalous dimensions are given by the pole parts (P.P.)

$$
\hat{I}=\frac{1}{\varepsilon} \hat{I}_{\text {P.P. }}+O\left(\varepsilon^{0}\right)
$$

of the respective integrals. The gluon operator, Eq. (6.8), reads in the momentum representation

$$
\begin{aligned}
O_{5}^{G}\left(\kappa_{1}, \kappa_{2}\right)= & \frac{1}{2} \tilde{x}^{\mu} \tilde{x}^{\mu^{\prime}}\left[F_{a \mu}{ }^{\nu}\left(\kappa_{1} \tilde{x}\right) \widetilde{F}_{\mu^{\prime} \nu}\left(\kappa_{2} \tilde{x}\right)-F_{\mu \nu}^{a}\left(\kappa_{2} \tilde{x}\right) \widetilde{F}_{\mu^{\prime}}{ }^{\nu}\left(\kappa_{1} \tilde{x}\right)\right] \\
= & \int \frac{d p_{1} d p_{2}}{4(2 \pi)^{2 N}}\left(e^{i \kappa_{1} \tilde{x} p_{1}-i \kappa_{2} \tilde{x} p_{2}}-e^{i \kappa_{2} \tilde{x} p_{1}-i \kappa_{1} \tilde{x} p_{2}}\right) \\
& \times \tilde{x}^{\mu} \varepsilon_{\mu \nu \rho \sigma} \tilde{x} p_{1} A_{a}^{\nu}\left(p_{1}\right)\left(p_{2}^{\rho} A_{a}^{\sigma}\left(p_{2}\right)-p_{2}^{\sigma} A_{a}^{\rho}\left(p_{2}\right)\right) .
\end{aligned}
$$

The one-loop correction to this operator is calculated. We work in the axial gauge, so that

$$
\begin{aligned}
& \tilde{x}^{\mu} F_{\mu \nu}(\kappa \tilde{x}) \rightarrow \tilde{x} \partial_{\kappa \tilde{x}} A_{\nu}(\kappa \tilde{x}), \\
& \tilde{x}^{\mu} \tilde{F}_{\mu \nu}(\kappa \tilde{x}) \rightarrow \tilde{x}^{\mu} \varepsilon_{\mu \nu \sigma \rho} \partial_{\kappa \tilde{x}}^{\sigma} A^{\rho}(\kappa \tilde{x})
\end{aligned}
$$

We are interested in pole-parts only, which for $\Delta K^{G q}$ correspond to the counter term of the quark operator. Actually not the complete gluon operator but only the term

$$
\tilde{x}^{\mu} \tilde{x}^{\mu^{\prime}} \tilde{F}_{\mu^{\prime}}^{\nu}\left(\kappa_{1} \tilde{x}\right) F_{\mu \nu}\left(\kappa_{2} \tilde{x}\right)
$$

needs to be used in the calculation which corresponds to the analytic expression:

$$
T\left(\tilde{x}^{\mu} \tilde{x}^{\mu^{\prime}} F_{\mu \nu}\left(\kappa_{2} \tilde{x}\right) \tilde{F}_{\mu^{\prime}}{ }^{\nu}\left(\kappa_{1} \tilde{x}\right) S\right)=\int \frac{d^{N} p_{1} d^{N} p_{2}}{(2 \pi)^{2 N}} \frac{1}{(2 \pi)^{N}} \bar{\psi}\left(p_{1}\right) \widehat{I}\left(p_{2}, p_{1}, \kappa_{2}, \kappa_{1}\right) \psi\left(p_{2}\right)
$$

with

$$
\widehat{I}\left(p_{2}, p_{1}, \kappa_{2}, \kappa_{1}\right)=e^{i \kappa_{1} \tilde{x} p_{1}-i \kappa_{2} \tilde{x} p_{2}} I_{1}\left(p_{1}, p_{2}, \kappa_{1}, \kappa_{2}\right)+e^{i \kappa_{2} \tilde{x} p_{1}-i \kappa_{1} \tilde{x} p_{2}} I_{2}\left(p_{2}, p_{1}, \kappa_{2}, \kappa_{1}\right)
$$

and

$$
\begin{aligned}
I_{1}= & \int d^{N} k\left(i g \gamma_{\mu}\right) i \frac{\hat{k}}{k^{2}}\left(i g \gamma_{\nu}\right) \frac{1}{i}\left(g^{\mu \sigma}-\frac{\tilde{x}^{\mu} q_{1}^{\sigma}+\tilde{x}^{\sigma} q_{1}^{\mu}}{\tilde{x} q_{1}}\right) \frac{1}{q_{1}^{2}} \frac{1}{i}\left(g^{\nu \rho}-\frac{\tilde{x}^{\nu} q_{2}^{\rho}+\tilde{x}^{\rho} q_{2}^{\nu}}{\tilde{x} q_{2}}\right) \frac{1}{q_{2}^{2}} \\
& \times\left(-i q_{1}^{\kappa} \tilde{x}^{\lambda} i\left(\tilde{x} q_{2}\right) \varepsilon_{\kappa \lambda \rho \sigma}\right) e^{i \kappa \tilde{x} k}
\end{aligned}
$$

where $q_{1}=p_{1}-k$ and $q_{2}=p_{2}-k . \quad I_{2}\left(p_{2}, p_{1}, \kappa_{2}, \kappa_{1}\right)$ is obtained by interchanging $\gamma^{\nu} \leftrightarrow \gamma^{\mu}$, $p_{1} \leftrightarrow p_{2}$ and $\kappa_{1} \leftrightarrow \kappa_{2}$. Here contrary to our convention adopted in the main part we use $\kappa=\kappa_{2}-\kappa_{1}=2 \kappa_{-}$, and the color factors are suppressed. The individual steps of the calculation are performed by a FORM-program 44:

(I) Firstly, the $\gamma$-algebra is evaluated. The result is represented by projections onto the matrices

$$
\mathbf{1}, \quad \gamma_{5}, \quad \gamma_{\mu}, \quad \gamma_{5} \gamma_{\mu}, \quad \sigma_{\mu \nu}, \quad \gamma_{5} \sigma_{\mu \nu}
$$


Here the only contribution is due to the axial vector part. The resulting expressions have been classified with the help of the pole-parts $J_{l m}^{\rho_{1} \ldots \rho_{n}}$ of basic integrals,

$$
\begin{aligned}
I_{1}=-\frac{i g^{2}}{4} \quad \gamma_{5} \gamma_{\mu} & \left\{2 J_{33}^{\mu \rho \sigma} \tilde{x}_{\rho} \tilde{x}_{\sigma}\right. \\
& +J_{32}^{\rho \sigma}\left[\tilde{x}^{\mu}\left(p_{1 \rho} \tilde{x}_{\sigma}+p_{2} \tilde{x}_{\sigma}\right)-\tilde{x}_{\rho} \tilde{x}_{\sigma}\left(p_{1}^{\mu}+p_{2}^{\mu}\right)-g_{\rho}^{\mu} \tilde{x}_{\sigma} \tilde{x}\left(p_{1}+p_{2}\right)\right] \\
& -J_{31}^{\rho}\left[\tilde{x}^{\mu}\left(p_{1 \rho} \tilde{x} p_{2}+p_{2 \rho} \tilde{x} p_{1}\right)-\tilde{x}_{\rho}\left(p_{1}^{\mu} \tilde{x} p_{2}+p_{2}^{\mu} \tilde{x} p_{1}\right)\right] \\
& \left.-2 J_{21}^{\rho} \tilde{x}^{\mu} \tilde{x}_{\rho}+J_{20} \tilde{x}^{\mu} \tilde{x}\left(p_{1}+p_{2}\right)\right\} .
\end{aligned}
$$

In the calculation of the pole-parts of the individual integrals we apply the $\alpha$-representation of the Feynman denominators and perform the momentum integrals. The exponentials are expanded into Taylor series as

$$
\exp (i \kappa k \tilde{x})=\sum_{n=0}^{\infty} \frac{(i \kappa k \tilde{x})^{n}}{n !}
$$

Because of $\tilde{x}^{2}=0$ only the first few terms of these expansions contribute.

(II) The terms $J_{l m}^{\rho_{1} \ldots \rho_{n}}$ in Eq. (B.9) are

$$
\begin{aligned}
& J_{33}^{\lambda \mu \nu}=\text { P.P. } \int d^{N} k \frac{k^{\lambda} k^{\mu} k^{\nu}}{q_{1}^{2} q_{2}^{2} k^{2}} e^{i k \tilde{x} \kappa} \\
& =\frac{i \pi^{2}}{2 \varepsilon} \int D_{0} \alpha e^{i \tilde{x} P(\alpha) \kappa}\left\{\left(g^{\lambda \mu} P^{\nu}(\alpha)+g^{\mu \nu} P^{\lambda}(\alpha)+g^{\nu \lambda} P^{\mu}(\alpha)\right)\right. \\
& +i \kappa\left(\tilde{x}^{\lambda} P^{\mu}(\alpha) P^{\nu}(\alpha)+\tilde{x}^{\mu} P^{\nu}(\alpha) P^{\lambda}(\alpha)+\tilde{x}^{\nu} P^{\lambda}(\alpha) P^{\mu}(\alpha)\right) \\
& -i \kappa \frac{D(\alpha)}{2}\left(g^{\lambda \mu} \tilde{x}^{\nu}+g^{\mu \nu} \tilde{x}^{\lambda}+g^{\nu \lambda} \tilde{x}^{\mu}\right) \\
& \left.-(i \kappa)^{2} \frac{D(\alpha)}{2}\left(P^{\lambda}(\alpha) \tilde{x}^{\mu} \tilde{x}^{\nu}+P^{\mu}(\alpha) \tilde{x}^{\nu} \tilde{x}^{\lambda}+P^{\nu}(\alpha) \tilde{x}^{\lambda} \tilde{x}^{\mu}\right)+c_{1} \tilde{x}^{\lambda} \tilde{x}^{\mu} \tilde{x}^{\nu}\right\}, \\
& J_{32}^{\mu \nu}=\text { P.P. } \int d^{N} k \frac{k^{\mu} k^{\nu}}{q_{1}^{2} q_{2}^{2} k^{2}} e^{i k \tilde{x} \kappa} \\
& =\frac{i \pi^{2}}{2 \varepsilon} \int D_{0} \alpha e^{i P(\alpha) \tilde{x} \kappa}\left\{\left(g^{\mu \nu}+i \kappa\left(P^{\mu}(\alpha) \tilde{x}^{\nu}+P^{\nu}(\alpha) \tilde{x}^{\mu}\right)+c_{2} \tilde{x}^{\mu} \tilde{x}^{\nu}\right\},\right. \\
& J_{31}^{\nu}=\text { P.P. } \int d^{N} k \frac{k_{\nu}}{q_{1}^{2} q_{2}^{2} k^{2}} e^{i k \tilde{x} \kappa}=\frac{i \pi^{2}}{2 \varepsilon} i \tilde{\kappa} \tilde{x}^{\mu} \int D_{0} \alpha e^{i \tilde{x} P(\alpha) \kappa}, \\
& J_{21}^{\nu}=\text { P.P. } \int d^{N} k \frac{k_{\nu}}{q_{1}^{2} q_{2}^{2}} e^{i k \tilde{x} \kappa} \\
& =\frac{i \pi^{2}}{\varepsilon} \int D_{0} \alpha \delta\left(1-\alpha_{1}-\alpha_{2}\right) e^{i \tilde{x} P(\alpha) \kappa}\left(P^{\nu}(\alpha)-\frac{i}{2} \kappa \tilde{x}^{\nu} D(\alpha)\right), \\
& J_{20}=\text { P.P. } \int d k \frac{1}{q_{1}^{2} q_{2}^{2}} e^{i k \tilde{x} \kappa_{-}}=\frac{i \pi^{2}}{\varepsilon} \int D_{0} \alpha \delta\left(1-\alpha_{1}-\alpha_{2}\right) e^{i \tilde{x} P(\alpha) \kappa}
\end{aligned}
$$

with the abbreviations

$$
\begin{aligned}
P^{\mu}(\alpha) & =p_{1}^{\mu} \alpha_{1}+p_{2}^{\mu} \alpha_{2} \\
D(\alpha) & =p_{1}^{2} \alpha_{1}\left(1-\alpha_{1}\right)+p_{2}^{2} \alpha_{2}\left(1-\alpha_{2}\right)-2 p_{1} p_{2} \alpha_{1} \alpha_{2}
\end{aligned}
$$


The constants $c_{1}$ and $c_{2}$ in Eqs. (B.11, B.12) remain unspecified here, since they do not contribute to the final result. Note that in the present case the two possible axial denominators $1 / k \tilde{x}$ are canceled by corresponding factors in the numerator. For completeness we note that in integrals with axial denominators, like

$$
\int d k \frac{k_{\mu} k_{\nu} e^{i k \tilde{x} \kappa}}{\left(p_{1}-k\right)^{2}\left(p_{2}-k\right)^{2} k^{2}}\left\{\frac{1}{k \tilde{x}}\right\},
$$

we proceed as follows. The integrand is first rewritten as

$$
\frac{e^{i k \tilde{x} \kappa}}{k \tilde{x}}=\frac{e^{i k \tilde{x} \kappa}-1}{k \tilde{x}}+\frac{1}{k \tilde{x}} .
$$

By applying the improved gauge prescription given by Leibbrandt and Mandelstam 45] the integrals decompose into a part being calculated by Leibbrandt already, whereas the other part, important for the operator renormalization, can be calculated without difficulties. For diagrams containing more than one axial dominator similar decompositions hold, as e.g.

$$
\begin{aligned}
e^{i k \tilde{x} \kappa} \frac{1}{(k \tilde{x})^{2}} \frac{1}{\mathrm{~N}} & =\frac{e^{i k \tilde{x} \kappa}-1-i k \tilde{x} \kappa}{(k \tilde{x})^{2} \mathrm{~N}}+\frac{1}{(k \tilde{x})^{2}} \frac{1}{\mathrm{~N}}+i \kappa \frac{1}{k \tilde{x}} \frac{1}{\mathrm{~N}} \\
e^{i k \tilde{x} \kappa} \frac{1}{(k \tilde{x})[(k-p) \tilde{x}]} \frac{1}{\mathrm{~N}} & =e^{i k \tilde{x} \kappa} \frac{1}{p \tilde{x}} \frac{1}{\mathrm{~N}}\left[\frac{1}{(k-p) \tilde{x}}-\frac{1}{k \tilde{x}}\right] \\
& =\frac{1}{p \tilde{x}} \frac{1}{\mathrm{~N}}\left[\frac{e^{i k \tilde{x} \kappa}-e^{i p \tilde{x} \kappa}}{(k-p) \tilde{x}}\right]+e^{i p \tilde{x} \kappa} \frac{1}{(k-p) \tilde{x}} \frac{1}{p \tilde{x}} \frac{1}{\mathrm{~N}} \\
& -\frac{1}{p \tilde{x}} \frac{1}{\mathrm{~N}}\left[\frac{e^{i k \tilde{x} \kappa}-1}{k \tilde{x}}+\frac{1}{k \tilde{x}}\right] .
\end{aligned}
$$

Here $\mathrm{N}$ denotes a standard Feynman denominator. As a result for the pole-part of $I_{1}$, Eq. (B.7), we obtain

$$
\begin{aligned}
I_{1}(\kappa)= & - \text { P.P. } \gamma_{5}(\gamma \tilde{x}) \frac{\pi^{2} g^{2}}{4} \int D_{0} \alpha e^{i \kappa \tilde{x} P(\alpha)}\left\{\delta\left(1-\alpha_{1}-\alpha_{2}\right)\left[2 P \tilde{x}(\alpha)-\tilde{x}\left(p_{1}+p_{2}\right)\right]\right. \\
& \left.+\left[i \kappa\left(\tilde{x} p_{1}\right)\left(\tilde{x} p_{2}\right)-2 \tilde{x} P(\alpha)-i \kappa(\tilde{x} P(\alpha))^{2}\right]\right\}
\end{aligned}
$$

(III) Now some appropriate identities are applied to cast the expressions into a form containing the operators again. In our case these rules can be derived by partial integration. We use the following relations:

$$
\begin{aligned}
& \int D_{0} \alpha e^{i \kappa \tilde{x} P(\alpha)}\left[(i \kappa \tilde{x} P(\alpha))^{2}\right]=\int D_{0} \alpha e^{i \kappa \tilde{x} P(\alpha)}\left[\delta\left(1-\alpha_{1}-\alpha_{2}\right)(i \kappa \tilde{x} P(\alpha)-3)+6\right] \\
& \int D_{0} \alpha e^{i \kappa \tilde{x} P(\alpha)} i \kappa \tilde{x} P(\alpha)=\int D_{0} \alpha e^{i \kappa \tilde{x} P(\alpha)}\left[\delta\left(1-\alpha_{1}-\alpha_{2}\right)-2\right] \\
& \int D_{0} \alpha e^{i \kappa \tilde{x} P(\alpha)} i \kappa \tilde{x} p_{1} \tilde{x} p_{2}=\int D_{0} \alpha e^{i \kappa \tilde{x} P(\alpha)}\left\{\delta\left(1-\alpha_{1}-\alpha_{2}\right)\left[\tilde{x} p_{2}-\frac{1}{i \kappa} \delta\left(\alpha_{1}\right)\right]\right. \\
&\left.+\frac{1}{i \kappa} \delta\left(\alpha_{1}\right) \delta\left(\alpha_{2}\right)\right\}(\mathrm{B} \\
& \int D_{0} \alpha e^{i \kappa \tilde{x} P(\alpha)} \delta\left(1-\alpha_{1}-\alpha_{2}\right) \alpha_{2}\left(\tilde{x} p_{1}-\tilde{x} p_{2}\right)=\int D_{0} \alpha e^{i \kappa \tilde{x} P(\alpha)} \frac{1}{i \kappa} \delta\left(1-\alpha_{1}-\alpha_{2}\right)\left(1-\delta\left(\alpha_{1}\right)\right) .
\end{aligned}
$$


(IV) As the result of this calculation we obtain

$$
I_{1}=\frac{1}{\varepsilon} \gamma_{5}(\tilde{x} \gamma) \frac{i \pi^{2} g^{2}}{4} \int D_{0} \alpha e^{i\left(\kappa_{2}-\kappa_{1}\right) \tilde{x}\left(p_{1} \alpha_{1}+p_{2} \alpha_{2}\right)} \frac{1}{\kappa_{2}-\kappa_{1}}\left\{\delta\left(\alpha_{1}\right) \delta\left(\alpha_{2}\right)-2\right\}+\text { finite terms } .
$$

Collecting all terms one gets

$$
\begin{array}{r}
\left(T O_{5}^{G}\left(\kappa_{1}, \kappa_{2}\right) S\right)=\frac{1}{2} \int \frac{d^{N} p_{1} d^{N} p_{2}}{(2 \pi)^{3 N}} \bar{\psi}\left(p_{1}\right)\left\{e^{i \kappa_{1} \tilde{x} p_{1}-i \kappa_{2} \tilde{x} p_{2}}\left[-I_{1}\left(p_{1}, p_{2}, \kappa_{1}, \kappa_{2}\right)+I_{1}\left(p_{1}, p_{2}, \kappa_{2}, \kappa_{1}\right)\right]\right. \\
\left.+e^{i \kappa_{2} \tilde{x} p_{1}-i \kappa_{1} \tilde{x} p_{2}}\left[-I_{2}\left(p_{2}, p_{1}, \kappa_{2}, \kappa_{1}\right)+I_{2}\left(p_{2}, p_{1}, \kappa_{1}, \kappa_{2}\right)\right]\right\} \psi\left(p_{2}\right)
\end{array}
$$

After inserting the explicit expressions and exploiting the symmetry relations of Eq. (B.25) one obtains finally

$$
\begin{aligned}
\text { P.P. }\left(T O_{5}^{G}\left(\kappa_{1}, \kappa_{2}\right) S\right)= & -\int \frac{d^{N} p_{1} d^{N} p_{2}}{(2 \pi)^{8}} i \bar{\psi}\left(p_{1}\right) \gamma_{5}(\tilde{x} \gamma) \psi\left(p_{2}\right) \\
& \times \frac{\pi^{2} g^{2}}{4(2 \pi)^{4}} \int D_{0} \alpha \frac{C_{F}}{\kappa_{2}-\kappa_{1}}\left\{\delta\left(\alpha_{1}\right) \delta\left(\alpha_{2}\right)-2\right\} \\
& \times\left\{e^{i \kappa_{1} \tilde{x} p_{1}-i \kappa_{2} \tilde{x} p_{2}}+e^{i \kappa_{2} \tilde{x} p_{1}-i \kappa_{1} \tilde{x} p_{2}}\right\} \\
& \times\left\{e^{i\left(\kappa_{2}-\kappa_{1}\right) \tilde{x}\left(p_{1} \alpha_{1}+p_{2} \alpha_{2}\right)}+e^{-i\left(\kappa_{2}-\kappa_{1}\right) \tilde{x}\left(p_{1} \alpha_{1}+p_{2} \alpha_{2}\right)}\right\} .
\end{aligned}
$$

Here we have introduced the color factor $C_{F}$. Now we introduce new variables $\kappa_{i}^{\prime}$, use the possibility of changing $\alpha_{1} \leftrightarrow \alpha_{2}$ and obtain

$$
\begin{aligned}
\text { P.P. }\left(T O_{5}^{G}\left(\kappa_{1}, \kappa_{2}\right) S\right)= & -\frac{\pi^{2} g^{2}}{4(2 \pi)^{4}} \int d \kappa_{1}^{\prime} d \kappa_{2}^{\prime} \int \frac{d^{N} p_{1} d^{N} p_{2}}{(2 \pi)^{8}} i \bar{\psi}\left(p_{1}\right) \gamma_{5}(\tilde{x} \gamma) \psi\left(p_{2}\right) \\
\times & \left\{e^{i \kappa^{\prime}{ }_{1} \tilde{x} p_{1}-i \kappa_{2}{ }_{2} \tilde{x} p_{2}}+e^{i \kappa^{\prime}{ }_{2} \tilde{x} p_{1}-i \kappa^{\prime}{ }_{1} \tilde{x} p_{2}}\right\} \int D_{0} \alpha \frac{C_{F}}{\kappa_{2}-\kappa_{1}}\left\{\delta\left(\alpha_{1}\right) \delta\left(\alpha_{2}\right)-2\right\} \\
\times & {\left[\delta\left(\kappa_{1}^{\prime}{ }_{1}-\left[\kappa_{1}+\alpha_{1}\left(\kappa_{1}-\kappa_{2}\right)\right]\right) \delta\left(\kappa_{2}^{\prime}-\left[\kappa_{2}-\alpha_{2}\left(\kappa_{1}-\kappa_{2}\right)\right]\right)\right.} \\
& \left.+\delta\left(\kappa_{1}^{\prime}-\left[\kappa_{1}-\alpha_{1}\left(\kappa_{1}-\kappa_{2}\right)\right]\right) \delta\left(\kappa_{2}^{\prime}-\left[\kappa_{2}+\alpha_{2}\left(\kappa_{1}-\kappa_{2}\right)\right]\right)\right] .
\end{aligned}
$$

Performing the momentum integration we finally end up with

$$
\begin{aligned}
\text { P.P. }\left(T O_{5}^{G}\left(\kappa_{1}, \kappa_{2}\right) S\right)= & -\int d \kappa^{\prime}{ }_{1} d \kappa^{\prime}{ }_{2} \frac{i}{2}\left[\bar{\psi}\left(\kappa_{1}^{\prime} \tilde{x}\right) \gamma_{5}(\tilde{x} \gamma) \psi\left(\kappa_{2}^{\prime} \tilde{x}\right)+\bar{\psi}\left(\kappa_{2}^{\prime}{ }_{2}\right) \gamma_{5}(\tilde{x} \gamma) \psi\left(\kappa_{1}^{\prime} \tilde{x}\right)\right] \\
& \times \frac{2 \pi^{2} g^{2}}{4(2 \pi)^{4}} \int D_{0} \alpha \frac{1}{\kappa_{2}-\kappa_{1}}\left[C_{F}\left\{\delta\left(\alpha_{1}\right) \delta\left(\alpha_{2}\right)-2\right\}\right] \\
& \times\left[\delta\left(\kappa_{1}^{\prime}-\left[\kappa_{1}+\alpha_{1}\left(\kappa_{1}-\kappa_{2}\right)\right]\right) \delta\left(\kappa_{2}^{\prime}-\left[\kappa_{2}-\alpha_{2}\left(\kappa_{1}-\kappa_{2}\right)\right]\right)\right. \\
& \left.+\delta\left(\kappa_{1}^{\prime}-\left[\kappa_{1}-\alpha_{1}\left(\kappa_{1}-\kappa_{2}\right)\right]\right) \delta\left(\kappa_{2}^{\prime}-\left[\kappa_{2}+\alpha_{2}\left(\kappa_{1}-\kappa_{2}\right)\right]\right)\right] .
\end{aligned}
$$

From this expression we can now read off the anomalous dimension,

$$
\Delta \widehat{K}_{0}^{G q}\left(\alpha_{1}, \alpha_{2}\right)=C_{F}\left[\delta\left(\alpha_{1}\right) \delta\left(\alpha_{2}\right)-2\right]
$$

Finally we would like to add a remark concerning the Feynman rules. It is important to note that for non-local operators the involved field operators appear with unrestricted momentum 
variables, e.g. $A^{\nu}\left(p_{1}\right)$, so that we are not enforced to determine operator vertices explicitly. We can simply apply the standard Feynman rules if we have in mind that the non-local operators are in fact $x$-space operators so that for diagrams in momentum space there remain certain exponential functions as relic terms from the lacking $\tilde{x}$-integration of the Fourier representation.

\section{The []$_{+}-$prescription}

The anomalous dimensions naturally appear in a regularized form, Eqs. (6.57-6.60) and 6.626.65) with the []$_{+}-$prescription given by (6.61). This prescription influences the subsequent relations, especially those of the evolution kernels $V_{\text {ext }}^{A A}\left(t, t^{\prime}, \tau\right)$. Let us consider the typical term

$$
V_{\text {sing }}\left(t, t^{\prime}, \tau\right)=\int D_{0} \alpha\left[\delta\left(\alpha_{1}\right)+\delta\left(\alpha_{2}\right)\right]\left[\frac{1}{\alpha_{1}+\alpha_{2}}\right]_{+} \delta\left(t-t^{\prime}\left(1-\alpha_{1}-\alpha_{2}\right)+\tau\left(\alpha_{1}-\alpha_{2}\right)\right)(\mathrm{C} .1)
$$

which appears in $V_{\mathrm{ext}}^{q q}=\Delta V_{\mathrm{ext}}^{q q}, V_{\mathrm{ext}}^{G G}$ and $\Delta V_{\mathrm{ext}}^{G G}$. We introduce the variables

$$
\alpha_{1}=\lambda \xi, \quad \alpha_{2}=\lambda(1-\xi)
$$

The corresponding measures are

$$
\int D_{0} \alpha\left[\delta\left(\alpha_{1}\right)+\delta\left(\alpha_{2}\right)\right]=\int_{0}^{1} d \lambda \int D \xi=\int_{0}^{1} d \lambda \int_{0}^{1} d \xi[\delta(\xi)+\delta(1-\xi)] .
$$

One obtains

$$
\begin{aligned}
V_{\text {sing }}\left(t, t^{\prime}, \tau\right) & =\int D \xi \int_{0}^{1} d \lambda\left[\frac{1}{\lambda}\right]_{+} \delta\left(t-t^{\prime}(1-\lambda)+\tau \lambda(2 \xi-1)\right) \\
& =\int_{0}^{1} d \lambda\left[\frac{1}{\lambda}\right]_{+}[\delta(T(\lambda)-\tau \lambda)+\delta(T(\lambda)+\tau \lambda)] \\
& =\int_{0}^{1} \frac{d \lambda}{\lambda}\left[\delta(T(\lambda)-\tau \lambda)+\delta(T(\lambda)+\tau \lambda)-2 \delta\left(t-t^{\prime}\right)\right],
\end{aligned}
$$

with $T(\lambda)=t-t^{\prime}(1-\lambda)$. The latter integral may now be rewritten in terms of the variable $x$ and $y$, Eq. (7.36), yielding

$$
\begin{aligned}
V_{\text {sing }}\left(t, t^{\prime}, \tau\right) & =\frac{1}{2|\tau|} \frac{1}{[y-x]_{+}}[\rho(x, y)-\rho(\bar{x}, \bar{y})] \\
& =\frac{1}{2|\tau|}\left[\frac{\rho(x, y)}{[y-x]_{+}}+\frac{\rho(\bar{x}, \bar{y})}{[\bar{y}-\bar{x}]_{+}}\right],
\end{aligned}
$$

where the []$_{+}-$prescription acts to the right.

\section{Sample calculation of extended evolution kernels}

This Appendix is devoted to show how the extended evolution kernels are obtained. As an example we consider $V_{\text {ext }}^{G q}\left(t, t^{\prime}, \tau\right)$. We evaluate Eq. (7.34)

$$
\begin{aligned}
V_{\mathrm{ext}}^{G q}\left(t, t^{\prime}, \tau\right) & =\int_{0}^{1} d \alpha_{1} \int_{0}^{1-\alpha_{1}} d \alpha_{2} \widehat{K}_{0}^{G q}\left(\alpha_{1}, \alpha_{2}\right) \int_{-\infty}^{+\infty} \frac{d\left(\kappa \tilde{x} p_{+}\right)}{2 \pi} \\
& \times\left(i \kappa \tilde{x} p_{+} t\right)^{-1} \exp \left\{i \kappa \tilde{x} p_{+}\left[t-\left(1-\alpha_{1}-\alpha_{2}\right) t^{\prime}+\tau\left(\alpha_{1}-\alpha_{2}\right)\right]\right\}
\end{aligned}
$$


with

$$
\widehat{K}_{0}^{G q}\left(\alpha_{1}, \alpha_{2}\right)=-C_{F}\left\{\delta\left(\alpha_{1}\right) \delta\left(\alpha_{2}\right)+2\right\} .
$$

Because of the scaling relation

$$
V_{\mathrm{ext}}^{A B}\left(t, t^{\prime}, \tau\right)=\frac{1}{\tau} V_{\text {ext }}^{A B}\left(\frac{t}{\tau}, \frac{t^{\prime}}{\tau}, 1\right),
$$

and reminding the general investigations in [5], we perform the calculations for $\tau=1$ and $0<x, y<1$. The relation of the variables $x$ and $y$ to $t, t^{\prime}$ and $\tau$ is given in Eqs. (7.36). The internal integral over $\kappa \tilde{x} p_{+}$yields

$$
\frac{1}{2 \pi} \int_{-\infty}^{+\infty} \frac{d\left(\kappa \tilde{x} p_{+}\right)}{i \kappa \tilde{x} p_{+}} \exp \left\{i \kappa \tilde{x} p_{+} X\right\}=\frac{1}{2}[\Theta(X)-\Theta(-X)]=\frac{1}{2} \varepsilon(X) .
$$

The latter equation is distribution-valued in the sense of tempered distributions [25, 34]. The l.h.s of Eq. (D.4) is the Fourier-transform of the distribution

$$
\frac{1}{2 \pi i} \mathrm{P} \frac{1}{z} \text {. }
$$

This distribution can be represented by

$$
\frac{1}{2 \pi i} \mathrm{P} \frac{1}{z}=\lim _{\varepsilon \rightarrow 0^{+}} \frac{1}{4 \pi i}\left[\frac{1}{z-i \varepsilon}+\frac{1}{z+i \varepsilon}\right] .
$$

From Eqs. (8.15a) and (5.17) follows then Eq. (D.4).

In terms of the variables $x, y$ we solve

$$
\begin{aligned}
t V_{\text {ext }}^{G q}\left(t, t^{\prime}, \tau\right)= & \int_{0}^{1} d \alpha_{1} \int_{0}^{1-\alpha_{1}} d \alpha_{2} \widehat{K}_{0}^{G q}\left(\alpha_{1}, \alpha_{2}\right) \\
& \frac{1}{2}\left\{\Theta\left[x-\left(1-\alpha_{1}-\alpha_{2}\right) y-\alpha_{2}\right]-\Theta\left(-\left[x-\left(1-\alpha_{1}-\alpha_{2}\right) y-\alpha_{2}\right]\right)\right\},
\end{aligned}
$$

where

$$
\begin{aligned}
\int D_{0} \alpha \Theta\left[x-\left(1-\alpha_{1}-\alpha_{2}\right) y-\alpha_{2}\right]= & \left(x-\frac{y}{2}\right)+\Theta(y-x) \frac{(y-x)^{2}}{2 y} \\
-\Theta(x-y) \frac{(\bar{y}-\bar{x})^{2}}{2 \bar{y}} & \\
\int D_{0} \alpha \Theta\left[x-\left(1-\alpha_{1}-\alpha_{2}\right) y-\alpha_{2}\right] \delta\left(\alpha_{1}\right) \delta\left(\alpha_{2}\right)= & \Theta(x-y) .
\end{aligned}
$$

Furthermore we observe that

$$
\int D_{0} \alpha \Theta\left(-\left[x-\left(1-\alpha_{1}-\alpha_{2}\right) y-\alpha_{2}\right]\right)=\int D_{0} \alpha \Theta\left(\bar{x}-\left(1-\alpha_{1}-\alpha_{2}\right) \bar{y}-\alpha_{2}\right) .
$$

Using these equations and taking into account the general structure of $V_{\mathrm{ext}}^{G q}(x, y)$ we obtain finally

$$
\begin{array}{r}
(2 x-1) V_{\text {ext }}^{G q}(x, y)=C_{F} \frac{1}{\tau}\left\{\Theta\left(\frac{x}{y}\right) \Theta\left(1-\frac{x}{y}\right) \operatorname{sign}(y)\left(1-\frac{x^{2}}{y}\right)\right. \\
\left.-\Theta\left(\frac{\bar{x}}{\bar{y}}\right) \Theta\left(1-\frac{\bar{x}}{\bar{y}}\right) \operatorname{sign}(\bar{y})\left(1-\frac{\bar{x}^{2}}{\bar{y}}\right)\right\} .
\end{array}
$$


Eq. (D.10) also allows to derive the splitting function in the case of forward Compton scattering. It is obtained in the the limit $\tau \rightarrow 0$ for $t^{\prime}>0$, with $\operatorname{sign}(y)=-\operatorname{sign}(\bar{y})=1$. Here the variables $x, y$ are expressed in terms of the variables $\left(t, t^{\prime}\right)$ and $\tau$, Eqs. (7.36). In this limit $x / y=\bar{x} / \bar{y} \rightarrow z=t / t^{\prime}$ holds. Thus $V_{\text {ext }}^{G q}\left(t, t^{\prime}, \tau\right)$ is given by

$$
\left.V_{\text {ext }}^{G q}\left(t, t^{\prime}, \tau\right)\right|_{t^{\prime}>0}=\frac{1}{t^{\prime} z} C_{F} \Theta(z) \Theta(1-z)\left\{2+\frac{2 t^{2}-4 t t^{\prime}}{2 t^{\prime 2}}\right\}+O(\tau)
$$

for small values of $\tau$, from which

$$
\left.\lim _{\tau \rightarrow 0} V_{\mathrm{ext}}^{G q}\left(t, t^{\prime}, \tau\right)\right|_{t^{\prime}>0}=\frac{1}{t^{\prime}} C_{F}\left(\frac{1+(1-z)^{2}}{z}\right) \Theta(z) \Theta(1-z)
$$

results. 


\section{References}

[1] K.G. Wilson, Phys. Rev. 179 (1969) 1699;

R.A. Brandt and G. Preparata, Fortschr. Phys. 18 (1970) 249;

W. Zimmermann, Lect. on Elementary Particle Physics and Quantum Field Theory, Brandeis Summer Inst., Vol. 1, (MIT Press, Cambridge, 1970), p. 395;

Y. Frishman, Ann. Phys. 66 (1971) 373.

[2] S.A. Anikin, M.C. Polivanov, and O.I. Zavialov, Fortschr. Physik 27 (1977) 459;

S.A. Anikin and O.I. Zavialov, Ann. Phys. (NY) 116 (1978) 135.

[3] O.I. Zavialov, Renormalized Feynman Diagrams, (Nauka, Moscow, 1979), in Russian;

Renormalized Quantum Field Theory (Kluwer Academic Press, Dordrecht, 1990).

[4] T. Ohrndorf, Nucl. Phys. 198 (1982) 26;

I.I. Balitsky Phys. Lett. 124B (1983) 230;

I.I. Balitsky and V.M. Braun, Proc. XXV LNPI Winter School on Physics, Leningrad (1990) p. 105.

[5] D. Müller, D. Robaschik, B. Geyer, F. Dittes, and J. Hořejši, Fortschr. Phys. 42 (1994) 2.

[6] M. Bordag and D. Robaschik, Nucl. Phys. B169 (1980) 445;

M. Bordag, B. Geyer, J. Hořejši and D. Robaschik, Zs. Phys. C26 (1985) 591;

B. Geyer, D. Müller, and D. Robaschik, preprint SLAC-Pub-6495 (1994).

[7] R. Gatto and G. Preparata, Nucl. Phys. B47 (1972) 313;

G. Altarelli and G. Preparata, Phys. Lett. 39B (1972) 371.

[8] A. De Rújula and E. De Rafael, Ann. Phys. (NY) 78 (1973) 132.

[9] E. Wieczorek, V.A. Matveev, and D. Robaschik, Teor. Mat. Fiz. 19 (1974) 14.

[10] A.V. Radyushkin, Phys. Lett. B380 (1996) 417.

[11] A.V. Radyushkin, Phys. Lett. B385 (1996) 333; Phys. Rev. D56 (1997) 5524.

[12] J. Blümlein, B. Geyer, and D. Robaschik, in : Proc. of the Int Workshop Deep Inelastic Scattering off Polarized Targets: Theory Meets Experiment, DESY Zeuthen, September 1997, Eds. J. Blümlein et al., (DESY, Hamburg, 1997), p. 196; DESY 97-209 (1997) and hep-ph/9711405.

[13] J. Blümlein, B. Geyer, and D. Robaschik, Phys. Lett. B406 (1997) 161 and Erratum.

[14] X. Ji, Nucl. Phys. B402 (1993) 217;

R. Jaffe and X. Ji, Nucl. Phys. B375 (1992) 527.

[15] D.J. Gross and S.B. Treiman, Phys. Rev. D4 (1971) 1059.

[16] M. Lazar, Konstruktion und Anwendung irreduzibler nichtlokaler Lichtkegeloperatoren in der Quantenchromodynamik, Diplomarbeit, Leipzig 1998.

[17] B. Geyer, M. Lazar, and D. Robaschik, hep-th/9901090. 
[18] I.I. Balitsky and V.M. Braun, Nucl. Phys. B311 (1988/89) 541.

[19] S. Wandzura and F. Wilczek, Phys. Lett. B72 (1977) 95.

[20] J. Blümlein and N. Kochelev, Phys. Lett. B381 (1996) 296; Nucl. Phys. B498 (1997) 285.

[21] C.G. Callan and D.J. Gross, Phys. Rev. Lett. 22 (1969) 156.

[22] R. Tarrach, Nuovo Cim. 28A (1975) 409.

[23] D. Drechsel, G. Knöchlein, A.Y. Korchin, A. Metz, and S. Scherer, Phys. Rev. C57 (1998) 941.

[24] W.A. Bardeen and Wu-Ki Tung, Phys. Rev. 173 (1968) 1423.

[25] I.M. Gel'fand and G.E. Schilow, Verallgemeinerte Funktionen (Distributionen), Vol. I, (Dt. Verlag d. Wissenschaften, Berlin, 1960);

Yu.A. Brychkov and A.P. Prudnikov, Integraltransforms of Generalized Functions (Nauka, Moscow, 1977), in Russian.

[26] J. Blümlein and A. Tkabladze, DESY 98-181, hep-ph/9812478, Nucl. Phys. B in print.

[27] J. Blümlein and A. Vogt, Phys. Rev. D58 (1998) 014020.

[28] T. Braunschweig, B. Geyer, and D. Robaschik, Ann. Phys. (Leipzig) 44 (1987) 407.

[29] T. Braunschweig, Einige Anwendungen der nichtlokalen Lichtkegelentwicklung auf die nichtvorwärts Streuung in der QCD (Singlett-Fall), PhD Thesis, Leipzig, 1984.

[30] J. Blümlein, V. Ravindran, W.L. van Neerven, and A. Vogt, DESY 98-036, hep-ph/9806368, in : Proc. of the 6th Int Workshop on Deep Inelastic Scattering and QCD, Brussels, April 1998, eds. Gh. Coremans and R. Rosen, (World Scientific, Singapore, 1998) 211.

[31] I.I. Balitsky and A.V. Radyushkin, Phys. Lett. B413 (1997) 114.

[32] D. Gross and F. Wilczek, Phys. Rev. D8 (1973) 3633; D9 (1974) 980;

H. Georgi and D. Politzer, Phys. Rev. D9 (1974) 416;

G. Altarelli and G. Parisi, Nucl. Phys. B126 (1977) 298.

[33] H. Ito, Prog. Theor. Phys. 54 (1975) 555;

K. Sasaki, Progr. Theor . Phys. 54 (1975) 1816;

M.A. Ahmed and G.G. Ross, Phys. Lett. B56 (1975) 385; Nucl. Phys. B111 (1976) 441.

[34] V.S. Vladimirov, Gleichungen der mathematischen Physik, (Dt. Verlag d. Wissenschaften, Berlin, 1972).

[35] F.-M. Dittes, D. Müller, D. Robaschik, B. Geyer, and J. Hořejši, Phys. Lett. B209 (1988) 325 .

[36] L. Mankiewicz, G. Piller, and T. Weigl, Eur. J. Phys. C5 (1998) 119;

L. Frankfurt, A. Freund, V. Guzey, and M. Strikman, Phys. Lett. B418 (1998) 345;

Erratum : B429 (1998) 414. 
[37] X. Ji, Phys. Rev. Lett. 78 (1997) 610, Phys. Rev. D55 (1997) 7114.

[38] G.P. Lepage and S.J. Brodsky, Phys. Lett. B87 (1979)359; Phys. Rev. D22 (1980) 2157;

S.J. Brodsky, Y. Frishman, G.P. Lepage, and C. Sachrajda, Phys. Lett. B91 (1980) 239.

[39] A.V. Efremov and A.V. Radyushkin, Phys. Lett. 94B (1980) 245; Teor. Mat. Fiz. 42 (1980) 97.

[40] M.K. Chase, Nucl. Phys. B174 (1980) 109;

T. Ohrndorf, Nucl. Phys. B186 (1981) 153;

M.A. Shifman and M.I. Vysotsky, Nucl. Phys. B186 (1981) 475;

M.V. Terentjev, Yad. Fiz. 33 (1981) 1692;

V.N. Baier and A.B. Grozin, Sov. J. Nucl. Phys. 35 (1982) 899; Nucl. Phys. B192 (1981) 476.

[41] I.M. Ryshik and I.S. Gradstein, Tables of Series, Products, and Integrals, (Dt. Verlag d. Wiss., Berlin, 1957).

[42] A.V. Radyushkin, hep-ph/9805342.

[43] A.S. Kronfeld and B. Nizic, Phys. Rev. D44 (1991) 3445.

[44] J. Vermaseren, FORM 2.3, (CAN, Amsterdam, 1996); AXODRAW, Comput. Phys. Commun. 83 (1994) 45.

[45] S. Mandelstam, Nucl. Phys. B201 (1983) 149;

G. Leibbrandt, Phys. Rev. D49 (1984) 1699; Rev. Mod. Phys. 59 (1987) 1067;

G. Leibbrandt, Noncovariant Gauges, (World Scientific, Singapore, 1994). 